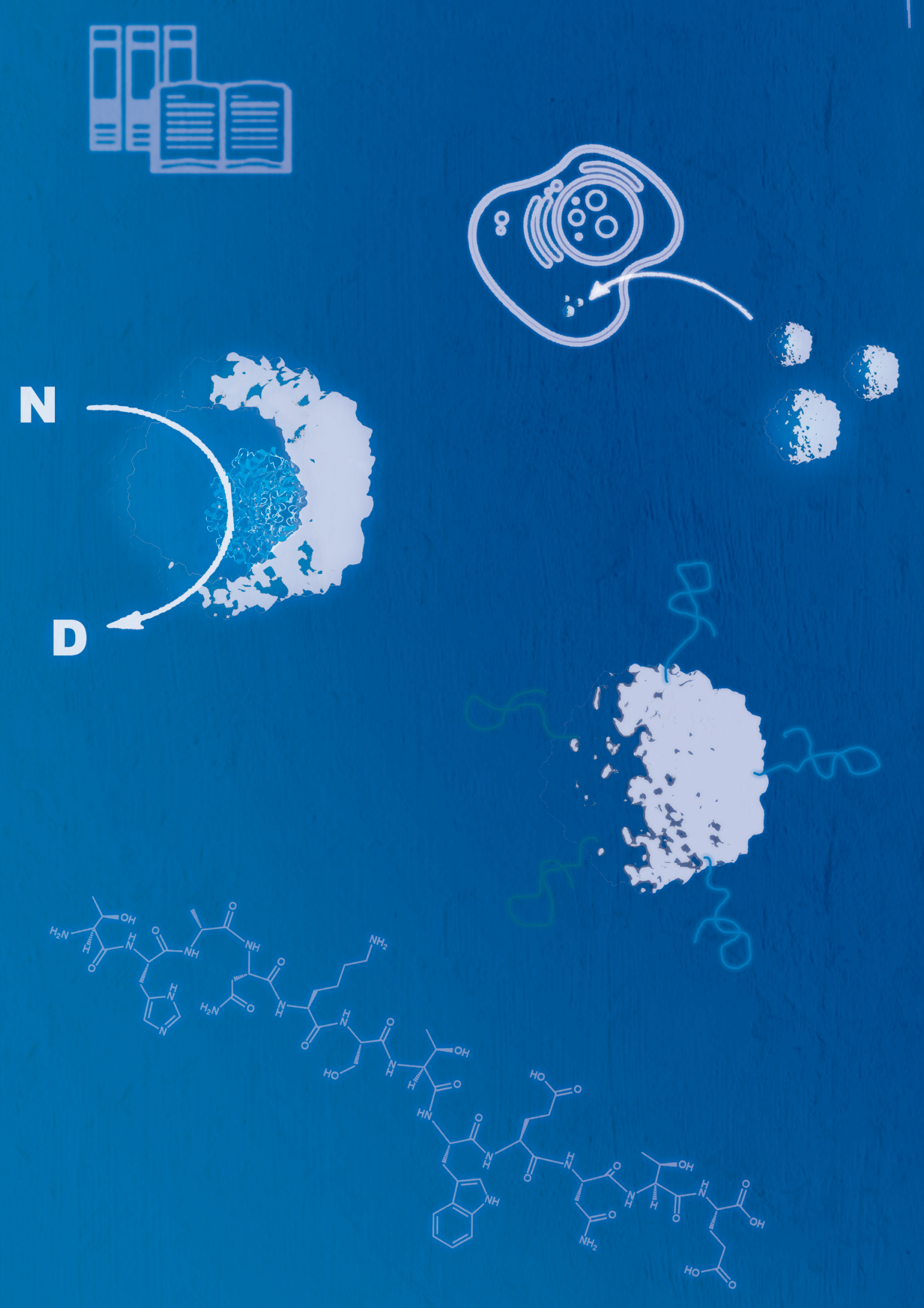

\title{
Encapsulins as Functional Protein Nanocages
}

Encapsulins as Functional Protein Nanocages

Public defense on Friday, $6^{\text {th }}$ of December 2019, at 16.45 in

the prof. dr. G. Berkhoffzaal (Waaier zaal 4), University of Twente, Enschede.

Prior to the defense I will give a short introduction to my thesis at 16.30. 


\section{ENCAPSULINS AS FUNCTIONAL PROTEIN NANOCAGES}

Robin Klem 


\section{Graduation committee:}

\section{Chairman}

Prof. dr. J. L. Herek

University of Twente

\section{Promotor}

Prof. dr. J. J. L. M. Cornelissen

University of Twente

\section{Members}

Prof. dr. J. Heddle

Jagiellonian University

Prof. dr. A. Kros

Leiden University

Prof. dr. ir. P. Jonkheijm

University of Twente

dr. ir. J. M. J. Paulusse

University of Twente

Prof. dr. P. C. J. J. Passier

University of Twente

The research described in this thesis was performed in the Biomolecular Nanotechnology (BNT) group, the MESA+ Institute for Nanotechnology and the Department of Science and Technology (TNW) of the University of Twente (UT). This research was supported by the European Research Council via the consolidator grant 'Protcage' (616907).

\section{MESA+}

Cover design: Luca Ricciardi, Mart Hoitink

Printer: Ipskamp Printing

ISBN: 978-90-365-4893-9

DOI: $10.3990 / 1.9789036548939$

Copyright (c) 2019, R. Klem, Enschede, Nederland. All rights reserved. 


\title{
ENCAPSULINS AS FUNCTIONAL PROTEIN NANOCAGES
}

\author{
PROEFSCHRIFT
}

ter verkrijging van

de graad van doctor aan de Universiteit Twente

op gezag van de rector magnificus

prof. dr. T. T. M. Palstra

volgens besluit van het College voor Promoties

in het openbaar te verdedigen

op vrijdag, 6 december 2019 om 16.45 uur

door

\section{Robin Klem}

geboren op 2 november 1991

te Wageningen, Nederland 
Dit proefschrift is goedgekeurd door:

Promotor

Prof. dr. Jeroen J. L. M. Cornelissen 


\section{Table of Contents}

1 General Introduction 1

1.1 General Introduction . . . . . . . . . . . . . . . . . 2

1.2 Aim and Outline of this Thesis . . . . . . . . . 2

1.3 References ..................... 5

2 Encapsulin: a New Protein Nanocage $\quad 7$

2.1 Protein Nanocages . . . . . . . . . . . . . . . . 8

2.2 Encapsulin General Introduction and Structure . . . . . . 9

2.3 Encapsulin Universality . . . . . . . . . . . . . . . . . . 14

2.4 Encapsulin Cargo and Function . . . . . . . . . . . 16

2.5 Encapsulin Production . . . . . . . . . . . . . . . 18

2.6 Encapsulin Modification and Application . . . . . . . . . . 19

2.7 Acknowledgements . . . . . . . . . . . . . . . 24

2.8 References . . . . . . . . . . . . . . . . 25

3 Cryo-EM Analysis of the Structure of Encapsulins 31

3.1 Introduction . . . . . . . . . . . . . . 32

3.2 Results and Discussion . . . . . . . . . . . . . . 33

3.3 Conclusions . . . . . . . . . . . . . . . . . . . . 45

3.4 Acknowledgements . . . . . . . . . . . . . . 45

3.5 Experimental Section . . . . . . . . . . . . . . . . . . . 46

3.6 References . . . . . . . . . . . . . . 53

4 Construction and Evaluation of L-Asparaginase loaded Protein Cages $\quad 59$

4.1 Introduction . . . . . . . . . . . . . . . . . 60

4.2 Results and Discussion . . . . . . . . . . . . . . 62

4.3 Conclusions . . . . . . . . . . . . . . . . . . . . . . . . 78

4.4 Acknowledgements . . . . . . . . . . . . . . . 79 
4.5 Experimental Section . . . . . . . . . . . . . . . . 79

4.6 References . . . . . . . . . . . . . . . . 87

5 Uptake, Retention and Immunogenicity of Brevibacterium linens Encapsulin in Cells $\quad 95$

5.1 Introduction . . . . . . . . . . . . . . . . 96

5.2 Results and Discussion . . . . . . . . . . . . . . . 97

5.3 Conclusions . . . . . . . . . . . . . . . . . . 115

5.4 Acknowledgements . . . . . . . . . . . . . . 115

5.5 Experimental Section . . . . . . . . . . . . . . 116

5.6 References . . . . . . . . . . . . . . . . 121

6 Exterior Modification of Thermotoga maritima Encapsulin with EETI-II Knottins 125

6.1 Introduction . . . . . . . . . . . . . . . 126

6.2 Results and Discussion . . . . . . . . . . . . . . . . 128

6.3 Conclusions . . . . . . . . . . . . . . . . . . . 137

6.4 Acknowledgements . . . . . . . . . . . . . . . 137

6.5 Experimental Section . . . . . . . . . . . . . . . 138

6.6 References . . . . . . . . . . . . . . . . 143

$\begin{array}{lr}\text { Summary } & 149\end{array}$

$\begin{array}{ll}\text { Samenvatting } & 153\end{array}$

$\begin{array}{ll}\text { List of Abbreviations } & 157\end{array}$

$\begin{array}{ll}\text { Acknowledgements } & 161\end{array}$

$\begin{array}{ll}\text { About the Author } & 167\end{array}$

List of Publications and Presentations 169

List of Publications . . . . . . . . . . . . . . . . . . . . . 169

List of Presentations . . . . . . . . . . . . . . . 170 


\section{Chapter 1}

\section{General Introduction}




\subsection{General Introduction}

In this thesis we explore encapsulins, a class of protein nanocages that was discovered in 1994 by Valdés-Stauber et al., ${ }^{1}$ further studied by Hicks et al. in $1998^{2}$ and that received increased attention after Sutter et al. published the structure of Thermotoga maritima encapsulin in $2008 .{ }^{3}$ Since then, a wide range of studies on the structure and potential application of encapsulins have been done.

Encapsulins are one of many different protein nanocages. ${ }^{4}$ These protein nanocages are very diverse in size, shape and function, which can be modified to create or change their function and have the potential to be applied for different purposes. These modifications include different cargo, targeting or stabilizing agents on the exterior and changes to the shape and size. ${ }^{5}$ The benefit of protein nanocages is that they are biodegradable and are, in theory, not toxic or dangerous and can be processed and excreted by the body. Virus-like protein nanocages can be extra beneficial given that their native purpose is to infiltrate cells. Thus, properties of nanoparticles as designed by nature are used to our advantage.

We focus on encapsulins, which have the added benefit of being highly stable protein nanocages. We study the initial steps to enable the use of these encapsulins in therapy.

\subsection{Aim and Outline of this Thesis}

Through the work presented in this thesis we aim to gain more insight in the recently discovered encapsulins. We expand on the present knowledge and explore future applications of encapsulins. 
Prior to any experimental chapters, Chapter 2 summarizes the published work that has been done on encapsulins prior to the publication of this thesis. This chapter is an outline on various aspects of encapsulins, including their structure, universality, cargo and modifications. This chapter is the foundation on which the following chapters are built.

Chapter 3 provides more insight in the structure of encapsulins. Studies have shown high genetical and structural similarity between different encapsulins, but detailed studies on the actual encapsulin structure are scarce. In Chapter 3, we study the structure of Brevibacterium linens encapsulins in high resolution, improving on the work done by Putri et $a l .{ }^{6}$ We relate this structure to the structure of other encapsulins (e.g. the encapsulins derived from Thermotoga maritima) and we analyse additional aspects of the encapsulin structure such as charge, flexibility and its polymeric building blocks.

We expand upon the effects of modified cargo in Chapter 4, where the native cargo of Brevibacterium linens encapsulins, dye decolorizing peroxidase, is changed to L-asparaginase. This new cargo is enzymatically active (it catalyzes the reaction of L-asparagine to L-aspartate) and can be used to treat acute lymphoblastic leukemia. In Chapter 4, we see that protein nanocages, in this case Brevibacterium linens encapsulin and the cowpea chlorotic mottle virus, can be modified to contain non-native cargo and that these nanocages can be applied in medically relevant settings. Chapter 4 also demonstrates that, depending on the protein nanocage, the effects are different and that thus for various applications, multiple protein nanocages should be studied.

While Chapter 4 contains a brief cell study, Chapter 5 expands on the interaction between encapsulins and cells. In this chapter we study the uptake, processing and cargo-protective properties of encapsulins in vari- 
ous cell types and we analyse the potential immunogenicity of encapsulins. It provides a foundation for further studies to the potential of encapsulins as drug delivery carriers.

In the previous chapters we primarily looked at the properties of the cargo and the protein shell of encapsulins, but we left one aspect unexplored: the modification and functionalization of the exterior of encapsulins. This principle is described in Chapter 6, where Thermotoga maritima encapsulins have been modified with a cysteine knot miniprotein that inhibits the activity of the protease trypsin. This modification can help to protect encapsulins from trypsin degradation if they were to be administered to the body, but also serves to demonstrate the potential to modify the encapsulin exterior. 


\subsection{References}

1. Valdés-Stauber, N.; Scherer, S. Isolation and Characterization of Linocin M18, a Bacteriocin Produced by Brevibacterium linens. Appl. Environ. Microbiol. 1994, 60, 3809-3814

2. Hicks, P. M.; Rinker, K. D.; Baker, J. R.; Kelly, R. M. Homomultimeric Protease in the Hyperthermophilic Bacterium Thermotoga maritima has Structural and Amino Acid Sequence Homology to Bacteriocins in Mesophilic Bacteria. FEBS Lett. 1998, 440, 393-398

3. Sutter, M.; Boehringer, D.; Gutmann, S.; Günther, S.; Prangishvili, D.; Loessner, M. J.; Stetter, K. O.; Weber-Ban, E.; Ban, N. Structural Basis of Enzyme Encapsulation into a Bacterial Nanocompartment. Nat. Struct. Mol. Biol. 2008, 15, 939-947

4. de Ruiter, M. V.; Klem, R.; Luque, D.; Cornelissen, J. J. L. M.; Castón, J. R. Structural Nanotechnology: Three-dimensional Cryo-EM and its use in the Development of Nanoplatforms for in vitro Catalysis. Nanoscale. 2019, 11, 4130-4146

5. de Ruiter, M. V. Thesis: Virus-Based Organelles; Enzyme and DNA-Based Virus Nanostructures and their Cellular Interactions. Chapter 5. University of Twente, Enschede, the Netherlands. 2019, 97-126 6. Putri, R. M.; Allende-Ballestero, C.; Luque, D.; Klem, R.; Rousou, K. A.; Liu, A.; Traulsen, C. H.; Rurup, W. F.; Koay, M. S. T.; Castón, J. R.; Cornelissen, J. J. L. M. Structural Characterization of Native and Modified Encapsulins as Nanoplatforms for in Vitro Catalysis and Cellular Uptake. ACS Nano 2017, 11, 12796-12804 
Chapter 1 


\section{Chapter 2}

\section{Encapsulin: a New Protein}

Nanocage

\section{Abstract}

Since Sutter et al. studied encapsulins in 2008, the amount of research on encapsulins has increased significantly. Encapsulins are a class of protein nanocages that have unique properties. In this review chapter, we explore the published research that has been done on the encapsulin protein nanoparticles. During this literature study, we found detailed information on the structure and universality of encapsulins, as well as information on the different encapsulin cargo. Additionally, we study the modifications, both of the cargo and the exterior, that have been made to encapsulins and the effects that these modifications have. In doing so, this chapter creates the foundation on which the following chapters are built.

The origins of this chapter are found in:

M. V. de Ruiter, R. Klem, D. Luque, J. J. L. M. Cornelissen, J. R. Castón; "Structural Nanotechnology: Three-dimensional Cryo-EM and its Use in the Development of Nanoplatforms for in Vitro Catalysis", Nanoscale, 2019, 11, 4130-4146 


\subsection{Protein Nanocages}

Protein nanocages are present in a large variety of organisms and come in different shapes, sizes and function. A general example are viruses, which consist of a protein shell encasing DNA or RNA. From these viruses researchers have made virus-like particles (VLPs), that have the protein shell but no longer contain the DNA or RNA. Additionally, other protein nanoparticles have been found such as bacterial microcompartments, ferritin and lumazine synthase. Each protein cage has a different function in different organisms. For example, ferritins store iron and are found in many different organisms, including humans. ${ }^{1}$ Bacterial microcompartments are protein shells found in bacteria that contain enzymes or enzyme cascades. ${ }^{2}$ Within these microcompartments, specific enzyme catalysed reactions occur that are separated from the rest of the bacterial cell; thus bacterial microcompartments protect the bacteria from potential toxins and metabolites. Lumazine synthase is found in multiple organisms and is an enzyme which itself can contain enzymatic cargo such as riboflavin synthase. ${ }^{3}$ The list of known protein cages is significantly longer than the examples provided here.

The diversity in function and particle morphology provides great potential for various applications that are not native to the protein nanocages; VLPs can be used to transport modified genes, ferritins to transport metallic ions or to function as a contrast agent, while bacterial microcompartments can contain non-native enzymes. Similar to the bacterial microcompartments, encapsulins are found in bacteria and can contain a protein as cargo. Thus, the protein nanocages can be used to alter the cell metabolism and can function as a nanoreactor in the target cell. In this chapter we focus on this protein nanocage. 


\subsection{Encapsulin General Introduction and Structure}

In 1994, Valdés-Stauber and Scherer described the isolation and characterization of a bacteriocin that was produced by the bacteria Brevibacterium linens. ${ }^{4}$ Four years later, Hicks et al. found a circular, homomultimeric protease that was purified from Thermotoga maritima. ${ }^{5}$ These discoveries remained relatively unnoticed until Sutter et al. further analysed these particles and labelled them encapsulins, which started the research on this new protein nanoparticle. ${ }^{6}$

Sutter et al. purified a protein nanocage from the T. maritima bacteria and derived a crystal structure from these protein cages (Figure 2.1), with which they could elucidate their function; the encapsulins and their cargo were involved in iron homeostasis. They found 'a spherical structure with an icosahedral $\mathrm{T}=1$ symmetry, a diameter of $240 \AA$ and a thickness of $\sim 25 \AA .{ }^{\prime 6}$ Other sizes and icosahedral encapsulins have been discovered since, such as the $300 \AA \mathrm{T}=3$ Myxococcus xanthus encapsulin nanoparticle. ${ }^{7}$ The T. maritima encapsulins consist of 60 monomers, with each monomer consisting of 3 domains: $\mathrm{P}, \mathrm{E}$ and $\mathrm{A}$. This is structurally analogous to the major capsid protein gp5 of the HK97 virus as well as that of the virus-like particle from Pyrococcus furiosus, which is discussed later in this chapter.

These three domains in the monomer each determine a part of the main structure: the P-domain contains the N-terminus and a conserved hydrophobic core; the E-domain is a loop that is the possible location of contact between two monomers; the A-domain contains the $\mathrm{C}$-terminus and is involved with the five-fold symmetry axis, where a pore is also located (among other locations). ${ }^{6}$ 


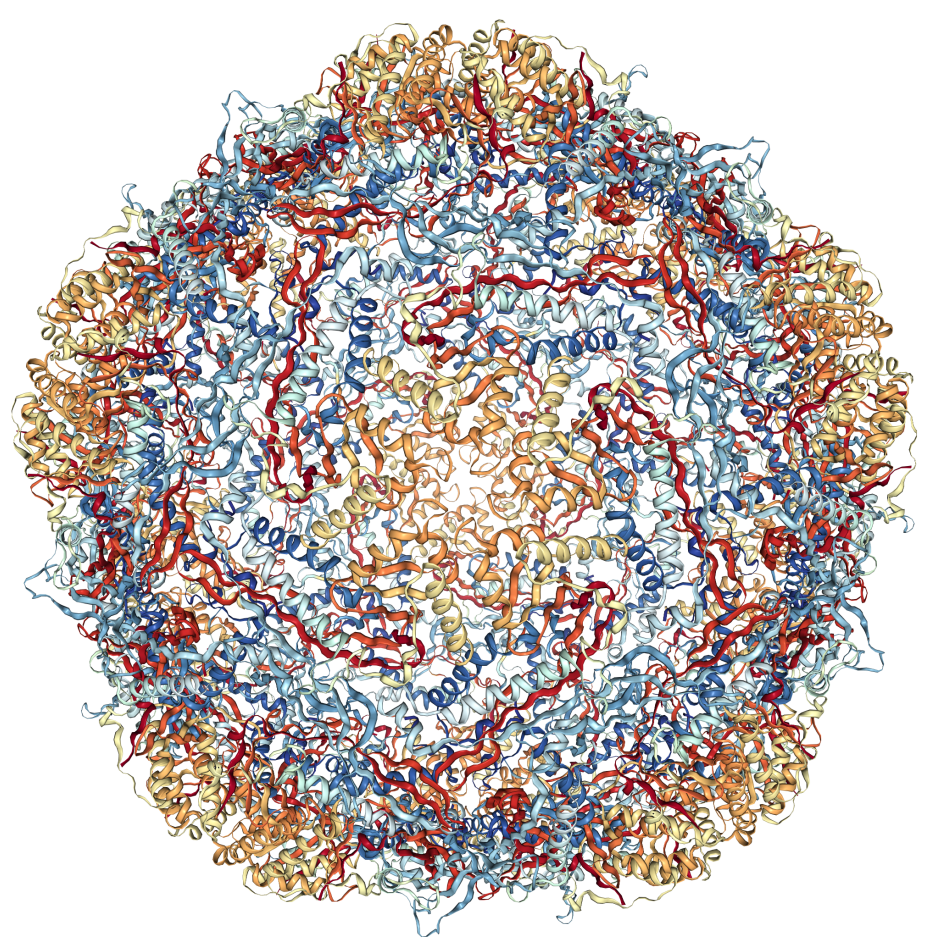

Figure 2.1: The crystal structure of Thermotoga maritima encapsulin as determined by Sutter et al. ${ }^{6}$ This image was taken from the RCSB Protein Data Bank, entry 3DKT.

These pores allow for transport of small molecules and ions. Rahmanpour et al. have shown that Rhodococcus jostii RHA1 encapsulins normally have pores smaller than $5 \AA$, which is similar to other encapsulins given their structural homology. ${ }^{8}$ While this is sufficient for ion transport, it could block the passage of larger molecules across the protein shell. However, these pores did allow the transport of the large dye molecule 2,2'azinobis(3-ethylbenzo-6-thiazolinesulfonic acid (ABTS), which is larger than $5 \AA$. Putri et al. confirmed that ABTS passes through the B. linens 
encapsulin membrane. ${ }^{9}$ This suggests that these pores are not fixed in size, but are actually flexible and dynamic. The extent of this dynamicity is yet to be studied and the effects of charge, hydrophobicity and size may all contribute to whether or not a molecule can pass through the encapsulin pores.

Williams et al. have engineered these pores in T. maritima encapsulins, which natively have pores sized $3 \AA{ }^{10}$ Using genetic modifications of areas around these pores, they were able to create pores ranging from 6 $11 \AA$ in size, while they retained the encapsulin structure. This occurred primarily when specific amino acids were deleted rather than replaced. Deletion did not result in deformed or non-forming particles, although it is probable that deleting amino acids has its limits as well. They proved that they had created larger encapsulin pores by demonstrating increased $\mathrm{Tb}^{3+}$ transport across the protein shell compared with native T. maritima encapsulin. Interestingly, they also found that trypsin addition did not break down the cargo protein, suggesting that the encapsulin shell protects the cargo protein.

The encapsulin structure is generally robust, which was demonstrated by Snijder et al. ${ }^{11}$ They also found that B. linens encapsulins that are loaded with cargo are structurally weaker than empty encapsulins, because the interaction of the cargo with the shell frustrates the shell by breaking local symmetry. They studied this phenomenon using nanoindentation and found a lower critical breaking force when the $B$. linens encapsulin was loaded with either the native dye decolorizing peroxidase (DyP) or non-native monomeric teal fluorescent protein (mTFP), compared with cargo-free encapsulins. The cargo-free encapsulins from both $B$. linens and T. maritima had the same critical breaking force, suggesting similar structural stability between the two encapsulins. ${ }^{11}$ They estimated 'a $\sim 1 \mathrm{Mcal} / \mathrm{mole}$ free energy required to induce structural collapse.' This 
suggests a mechanically rigid and resilient shell, that is both elastic and robust.

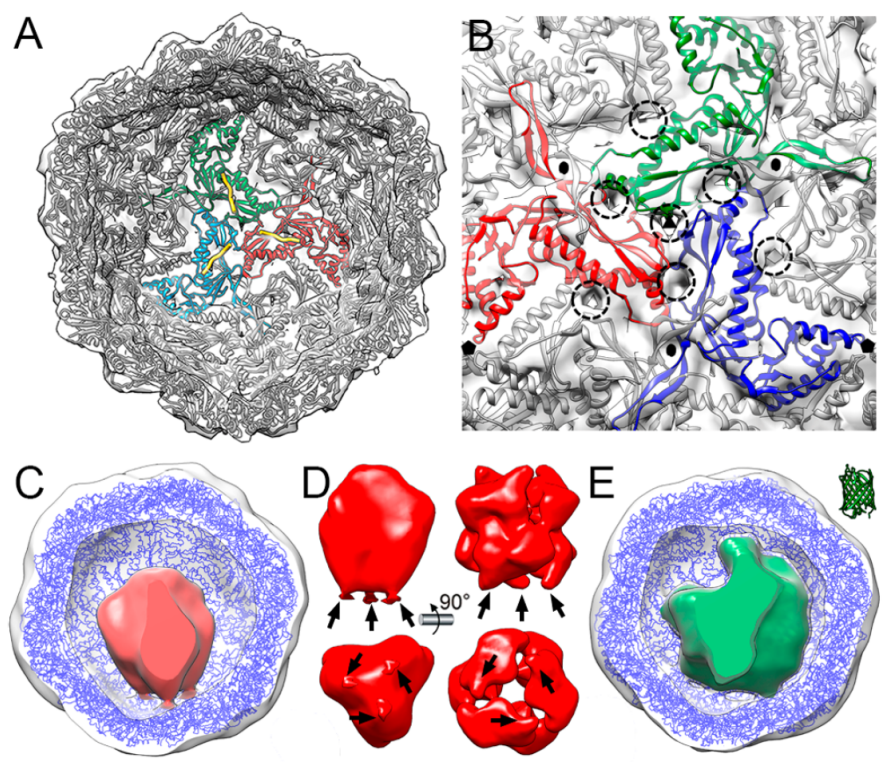

Figure 2.2: The crystal structure of Thermotoga maritima encapsulin has a good fit with the cryo-EM structure of Brevibacterium linens encapsulins. (A) the whole of the B. linens encapsulin in white, with in grey the overlay of the T. maritima crystal structure. The T. maritima monomers are highlighted in green, red and blue. (B) is a zoom of these highlighted monomers. (C) shows the B. linens encapsulin with its native DyP cargo (red) and T. maritima crystal structure overlay in blue, with the cargo and its binding sites highlighted in (D). (E) displays the B. linens encapsulin with modified cargo; mTFP. This image was created by Putri et al. ${ }^{9}$ and reprinted with the permission from ACS. Copyright ACS, 2017. 
Putri et al. demonstrated that empty encapsulins are not really empty, but contain some unidentified cargo. ${ }^{9}$ They studied different cargo in $B$. linens (DyP, mTFP and no cargo) and found small differences to the encapsulin shell structure, suggesting that the cargo binding to the encapsulin affects the structure. They also showed that encapsulins remain stable under high and low $\mathrm{pH}$, various salt concentrations and increased temperature. The structure of T. maritima can be fitted to the $B$. linens 3D map (Figure 2.2), which suggests that the nanoparticles might have similar origins, which is elaborated upon in later in this chapter.

The thermostability is further verified by the fact that encapsulins are found in thermophilic bacteria such as T. maritima. These bacteria are found in water with temperatures ranging from 55 to $90^{\circ} \mathrm{C}$, temperatures at which most proteins denature. ${ }^{12}$ The harsh environments that are present within the encapsulins, such as the presence of reactive oxygen species (ROS) and high concentrations of iron-ions (elaborated upon further in this chapter), also show that encapsulins are stable in extreme environments.

Because encapsulins are protein nanocages, they may be susceptible to proteases, which was shown by Valdés-Strauber et al. ${ }^{4}$ They also proved that addition of nucleases, lipases, catalases, $\alpha$-amylases and the proteases ficin and papain did not affect encapsulin structure, suggesting that $B$. linens encapsulins are robust and resilient against these proteases. Additional studies regarding temperature, long-term storage and $\mathrm{pH}$ changes showed that encapsulins are stable, which is important when applying encapsulins in medicine and industry. 


\subsection{Encapsulin Universality}

Encapsulins have been found in fifteen bacterial and two archaeal phyla. The initial discovery linked to the function was shown by Sutter et al. ${ }^{6}$ They found that the genetic sequence encoding for these protein nanoparticles shows high similarity between different bacteria, with large parts of the sequence conserved. Giessen et al. elaborated on this genetic and structural similarity by comparing more than 900 encapsulin genes ${ }^{13}$ and Putri et al. further demonstrated the structural similarity by fitting the crystal structure of T. maritima encapsulin to the cryo-EM derived 3D structure of B. linens. ${ }^{9}$

In their comparative study, Giessen et al. found that structurally, encapsulins can be categorized into 4 groups; $\mathrm{T}=1$; $\mathrm{T}=3$; ferritin-like protein (Flp)-fusions; and T3-like particles, with the majority belonging to the $\mathrm{T}=1$ or $\mathrm{T}=3$ groups. This categorization correlates to the cargo of the encapsulins. When encapsulating peroxidases or haemerythrin, encapsulins mostly form $\mathrm{T}=1$ protein cages, while iron-mineralizing encapsulin-associated firmicute (IMEF) cargo results in $\mathrm{T}=3$ nanoparticles. For the Flp-fusions, nanoparticles with both $\mathrm{T}=1$ and $\mathrm{T}=3$ icosahedral conformation are found. ${ }^{13}$

Besides the structure, the cargo of encapsulins is also non-diverse. Out of the 900 encapsulins, Giessen et al. identified 6 groups of protein cargo. For the majority of these groups, the cargo is either involved with iron storage and mineralization or the cargo is a peroxidase. Both types require compartmentalization, as large concentrations of iron-ions or ROS resulting from peroxidase-catalysed reactions can damage the bacteria if not contained. ${ }^{13}$ 

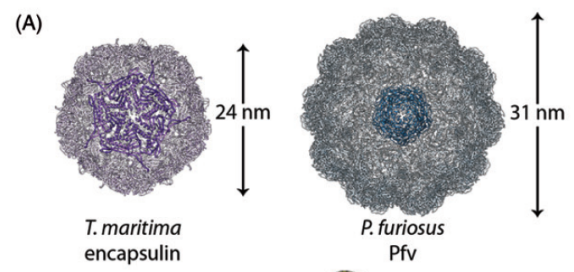

(B)
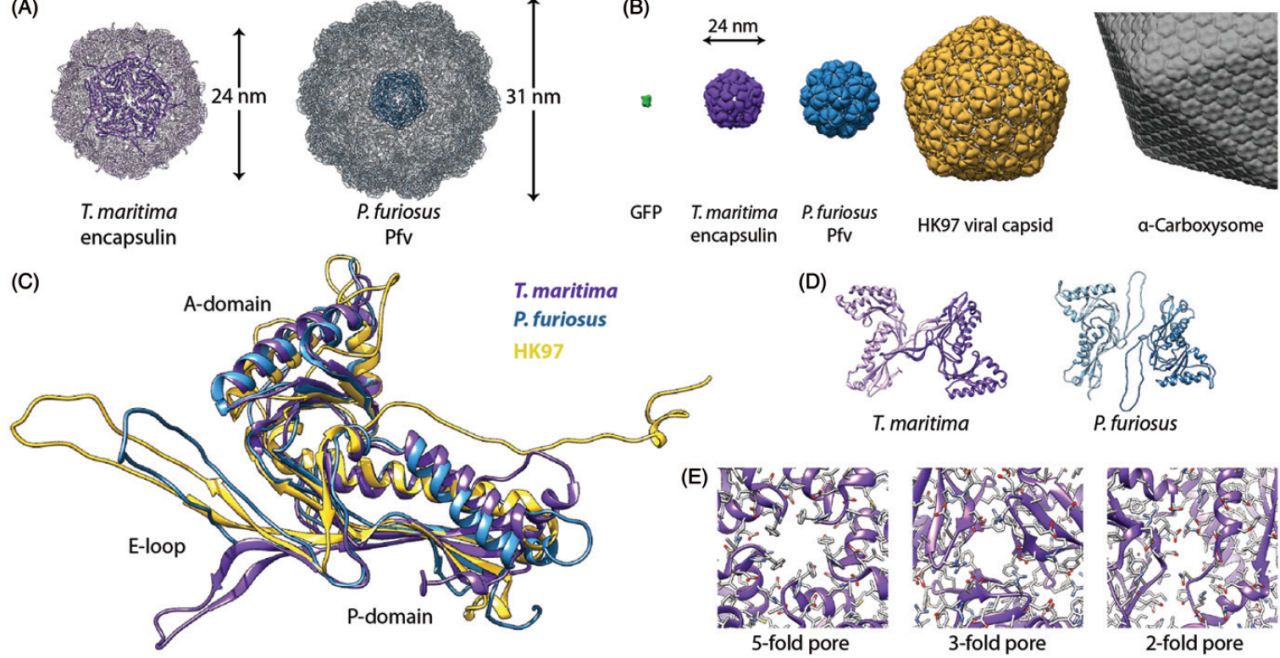

(D)
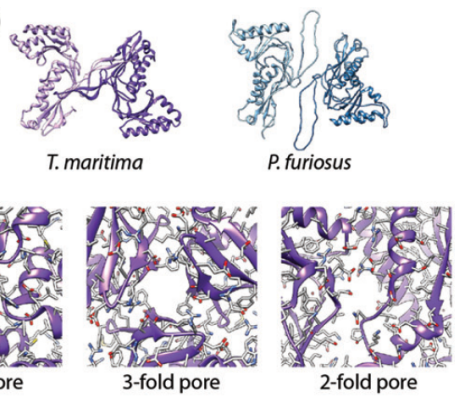

Figure 2.3: The structure of the encapsulin resembles that of known viruslike particles. (A) and (B) show the Thermotoga maritima encapsulin compared with other protein nanocompartments that are created from similar monomers. (C) demonstrates the similarity in the structure of the monomers from T. maritima encapsulins and the virus-like particles derived from Pyrococcus furiosus and HK97. The dimer interaction of $T$. maritima monomers and $P$. furiosus monomers is demonstrated in (D), while (E) shows the pores found in T. maritima encapsulins at the various axes. This image was created by Nichols et al. ${ }^{15}$ and reprinted with the permission from Taylor and Francis. Copyright Taylor and Francis, 2017.

In addition to the structural similarity, the genetic sequence encoding for the C-terminus of the cargo protein of various encapsulins is also maintained. ${ }^{7}$ This sequence is required for efficient cargo loading. Given that the encapsulins are structurally similar and that this C-terminus sequence likely interacts with the protein shell, the C-terminus of the cargo proteins is also conserved. Künzle et al. used the C-terminal peptide sequence from the T. maritima Flp cargo, coupled it to a gold nanoparticle and encapsulated this construct in encapsulins. ${ }^{14}$ They showed that 
while the peptide-tag is not required for encapsulation (it can be done based on charge alone), it is required for particle formation under high salt concentrations. This and other research, such as that from Putri et al. ${ }^{9}$ demonstrate that the C-terminal tag can be used to encapsulate non-native cargo.

The genetic, structural and cargo similarity between the hundreds of encapsulins suggests a common origin. When comparing the structure of the encapsulins and their monomers with the HK97 bacterial virus and the virus-like particles derived from $P$. furiosus, ${ }^{7}$ it is evident that there are structural similarities (Figure 2.3). ${ }^{15}$ This suggests that encapsulins originated from bacterial viruses and over time became bacterial nanocompartments.

\subsection{Encapsulin Cargo and Function}

Most native and modified encapsulin cargo contains an affinity tag on the C-terminus that stimulates encapsulation. This interaction has been studied by Cassidy-Amstutz et al., showing that there is a minimal peptide tag required for encapsulation of the protein cargo. ${ }^{16}$ With this minimal tag they were also able to load cargo into encapsulin using an in vitro method, showing that this tag was necessary. Rahmanpour et al. also showed this retention of the $\mathrm{C}$-terminus sequence in various bacterial DypB (native encapsulin cargo of $R$. jostii encapsulin) homologues, with a C-terminal sequence of 10 amino acids being strongly conserved. ${ }^{8}$ However, Künzle et al. encapsulated positively charged gold nanoparticle based on charge alone, without the need for the peptide tag. A highly positively charged cargo could be encased in encapsulins, which has a net negative charge on its interior, but only in low salt concentrations. In high salt concentrations, however, the tag was required to induce encapsulation of the cargo. $^{14}$ 
As discussed before, encapsulins have different native functions, but the variety is limited. The primary native cargo consists of either a peroxidase, like the DyP in B. linens encapsulin, ${ }^{6}$ or proteins that affect iron homeostasis, ${ }^{17}$ such as Flp (T. maritima) and IMEF. ${ }^{18}$ This is however not all encapsulin functionality, as Giessen et al. have also demonstrated encapsulin involvement in carbon fixation, oxidative and nitrosative stress resistance and anaerobic ammonium oxidation, ${ }^{13}$ highlighting the broad range of encapsulin functions.

Some encapsulins contain more than one type of enzyme, such as the Mycobacterium tuberculosis encapsulins that package three enzymes; a DyP, a bacterioferritin (BfrB) and a dihydroneopterin aldolase (FolB). These all have antioxidant properties, suggesting a role in oxidative stress response. ${ }^{19}$ The containment of multiple enzymes in a confined space could increase the reaction rates of the catalysed reactions because the increased local concentration of the substrates can influence the metabolic pathways.

All these cargo proteins deal with environments that are normally hazardous to an organism. The substrates and products in peroxidase catalysed reactions are often toxic (ROS), while large concentrations of iron ions are also unwanted in a cell. Thus, the compartmentalization of these products prevents damage to the cell while it is still able to perform these reactions or maintain iron homeostasis. 


\subsection{Encapsulin Production}

Encapsulins are genetically encoded in bacteria, often in an operon that also contains their cargo protein(s) including the sequence for the peptide tag. This principle can be used to produce encapsulins in Escherichia coli bacteria or yeast. This operon can be expressed within these organisms resulting in self-assembled, monodisperse protein nanocages that have encapsulated their cargo, which in turn can be purified from the encapsulin producing organism.

The formation of encapsulins is hard to study as it happens within the bacteria, making analysis difficult. Because of the previously described stability, it is also difficult to have the encapsulins disassemble and reassemble to study the formation, which can be done with other protein nanocages such as the cowpea chlorotic mottle virus (CCMV). It is only possible to disassemble encapsulins at low $\mathrm{pH}$ conditions $(\mathrm{pH} \mathrm{2})$, which is not the natural environment in bacteria, as demonstrated by Künzle et $a .^{14}$ The encapsulins appeared to reassemble to their native structure upon raising the $\mathrm{pH}$. Snijder et al. elaborated on the formation of encapsulins. They found that incomplete 58-mer encapsulins still encapsulate cargo, suggesting that the capsid formation is driven by the cargo and that the formation is driven by addition of dimers instead of monomers to the proto-encapsulin protein shell. ${ }^{11}$ They proposed a mechanism in which first dimers form and these dimers subsequently join together forming the encapsulin nanocage. We elaborate upon the formation of the encapsulin nanoparticle in Chapter 3.

This cargo driven particle formation can be related to the affinity tag that is found in most cargo proteins, except for the $P$. furiosus encapsulin where the cargo is fused directly to the encapsulin protein. While the affinity tag is important for cargo encapsulation, the exact method of interaction between the tag and the encapsulin monomer is not fully un- 
derstood, but is elaborated on by Sutter et al. ${ }^{6}$ They suggest that leucine and isoleucine found in the tag are buried in a hydrophobic pocket in the interior of the encapsulin shell. Additionally, encapsulins contain some material, even when no cargo is coded in the genetic sequence, further suggesting a cargo driven particle formation. ${ }^{9}$

When during the production encapsulins are expressed in E. coli, the cargo can be altered by genetically removing the sequence that codes for the native cargo and introducing a new cargo sequence, while retaining the C-terminal peptide tag. ${ }^{6}{ }^{20}$ This has been done with for example $B$. linens encapsulin, into which fluorescent proteins were introduced. ${ }^{9}$ These encapsulins can still be produced with equal yield compared with encapsulins that contain native cargo.

\subsection{Encapsulin Modification and Applica- tion}

Some encapsulins natively contain enzymes, such as DyP. Using this enzyme, enzymatic cascades can be created, such as the glucose oxidase - peroxidase cascade, which was demonstrated by Putri et al. using $B$. linens encapsulins. ${ }^{9}$ In this setting, the glucose oxidase was not encapsulated, but the product of the glucose oxidase catalysed reaction (peroxide) entered the encapsulin together with the added ABTS. The ABTS was further converted by the encapsulated peroxidase in the presence of the peroxide. Peroxidase activity has also been shown in $R$. jostii encapsulins by Rahmanpour et al. ${ }^{8}$

Encapsulins have also been modified to contain different protein cargo, which was demonstrated by Contreras et al. ${ }^{19}$ This can have benefits such as the co-localization and potential stabilization and protection of the cargo protein, which all could increase protein activity and longevity. 
Lau et al. demonstrated this protection using the fluorescent protein cargo mNeonGreen, which was protected from proteasome-based degradation in the yeast Saccharomyces cerevisiae. ${ }^{21}$ In addition, encapsulinbased nanoreactors co-packaged with the split Venus components Ven-N and Ven-C, with high loading yields, became fluorescent, demonstrating the co-localization. This was further explored by encapsulating Aro10p, an enzyme involved in tyrosine catabolism that might allow pro-drugs to be activated in the body. The $5 \AA$ pores allowed small molecule diffusion and the enzyme showed the anticipated decarboxylation activity. The cargo was again protected from protease degradation.

The modification of cargo and potential application was also shown by $\mathrm{Pu}-$ tri et al., who demonstrated that the encapsulin cargo could be changed from its native DyP to TFP and that the TFP was taken up in J774 macrophage cells, giving the encapsulins a cell staining function. ${ }^{9}$ Rurup et al. demonstrated with mass spectrometry that encapsulins can contain $\sim 12$ dimeric TFP cargo proteins per cage. ${ }^{11,20}$ This takes up more space than the native DyP cargo, demonstrating that encapsulins have the space to contain multiple or larger proteins. The fluorescent labelling is useful for staining purposes, but primarily demonstrated the potential to alter the protein cargo of encapsulins to a non-native protein.

Sigmund et al. demonstrated the encapsulation of non-native enzymes by encapsulating an engineered peroxidase, polymerized diaminobenzidine (APEX2), in encapsulins. ${ }^{22}$ This enzyme can be used for cellular electron microscopy (EM) imaging and proximity labelling. The system was expanded to encapsulate cystathionine $\gamma$-lyase which, in the presence of L-cysteine, catalyses the conversion of cadmium acetate in aqueous solution into cadmium sulfide nanocrystals. These nanocrystals were confined in the nano-sized interior of the encapsulins and generated a photoluminescence signal under UV-light. 
Other enzymatic cargo has also been encapsulated; Sigmund et al. encapsulated tyrosinase while retaining its activity, ${ }^{22}$ while Tamura et al. encapsulated luciferase in Rhodococcus erythropolis encapsulins. ${ }^{23}$ These enzymes were still active, indicating that they fold correctly and remain correctly folded when encapsulated. Additionally, the addition of the affinity tag did not nullify the enzyme activity. It also demonstrated that enzymes can be encapsulated that might be relevant for medicinal use. We expand on this principle in Chapter 4, where we show that Lasparaginase can be encapsulated while retaining its activity.

Other than protein cargo, Giessen et al. used encapsulins to store iron, showing that encapsulins can also contain metal ions, which is a known feature of the ferritin nanoparticle. ${ }^{24} \mathrm{He}$ et al. showed, using encapsulins from $M$. xanthus, that encapsulins can also encase the ferritin nanoparticle, which in turn functions as iron storage. ${ }^{25}$ This iron storage functionality was also studied by Sigmund et al. for the creation of ironbiomineralizing nanocompartments. ${ }^{26}$

Furthermore, Giessen et al. fused an AG4 peptide to the N-terminus of the encapsulin monomers, making this peptide point inwards. In this way, silver nanoparticles were encapsulated in the protein cage. The silver ions that were released from this complex were used as an antibacterial agent. ${ }^{27} \mathrm{~A}$ similar principle has been utilized by Künzle et al. to encapsulate gold nanoparticles, ${ }^{14}$ where they provided more insight into the binding of the affinity sequence to the encapsulin. They coupled a 7 amino acid peptide sequence (similar to the peptide tag used with the native cargo) to the gold nanoparticles. This tag interacts with a binding site in the interior of the encapsulin, located on the connecting point of two monomers that form a dimer. This provided more insight in the actual connection of particles in encapsulin. Additionally, they showed 
that particles could be loaded in encapsulin based on charge; the interior of encapsulin contains negatively charged patches, causing a positively charged cargo to be encapsulated. While this principle to encapsulate proteins is limited given that the encapsulins needed to disassemble at pH 2 prior to encapsulation of the cargo, it does open up the possibility to encapsulate other materials, which in turn can be utilized in various applications, for example to treat enzyme deficiency diseases, catalyse reactions or kill specific organisms or cells.

For the use of encapsulins as a drug delivery carrier, it is beneficial to target specific cells. This targeting can be achieved by modifying the exterior of encapsulins. Most encapsulins contain lysines, glutamic and aspartic acids or, like T. maritima encapsulins, cysteine amino acids with reactive groups on the exterior which can be utilized for chemical modifications. T. maritima is specifically interesting as it has 60 outward facing thiol-groups on its exterior (and another 60 that theoretically are exposed, but are more sterically hindered), that can be modified to create a homologous modified functional surface. Using coupling molecules such as the EMCS-linker or NHS-ester, other proteins, dyes, or targeting ligands can be bound to the encapsulin exterior. While not used for targeting purposes but for increasing the encapsulin stability, we explore this chemical modification of the T. maritima encapsulin exterior in Chapter 6.

As an alternative to the chemical modifications, the genetic sequence of encapsulins can be altered to include targeting loops or other moieties on their exterior. As shown in Figure 2.1, there are loops on the exterior of the encapsulin that could be used to insert an amino acid sequence with a specific function. This principle has been utilized by Moon et al. to create encapsulins with targeting peptides to specifically target hepatocellular carcinoma cells and Squamous cell carcinoma (SCC-7) cells. ${ }^{28,} 29$ 
With this SP94 peptide on the exterior, they transported doxorubicin as cargo attached to encapsulins, thus successfully killing HepG2 cancer cells. With an FcBP peptide, they also showed the specific targeting of fluorescently labelled FcBP-modified encapsulins to the SCC-7 cells, while no uptake was found in other studied cell types. We further study encapsulin uptake by various cells in Chapter 5 .

Genetic modification of encapsulins has also been utilized by inserting a genetic sequence for a SpyTag peptide in the encapsulin genetic sequence. ${ }^{30}$ Bae et al. used this to couple multiple proteins to the SpyTag peptide using the SpyTag-SpyCatcher system. These proteins were fluorescent proteins that target affibody ligands that were fused to the SpyCatcher, which in turn was coupled to the SpyTag. This created multifunctional nanoparticles with the potential to create a wide range of functionality. Choi et al. used genetic insertion to present an OT-1 peptide (SIINFEKL) to induce an immune response, like a vaccine. ${ }^{31}$ When mice immunized with OT-1 were infused with B16-OVA melanoma cells, a specific immune response was detected, suggesting that this 'vaccine' worked.

The principle to modify the surface for vaccination purposes was also used by Lagoutte et al. to create a vaccine based on encapsulin nanoparticles, this time modified with the M2e epitope. ${ }^{32}$ They showed that, in conjunction with the immune response against the epitope, there was an immune response against the cargo protein: green fluorescent protein. This suggests that encapsulins can be used as a carrier of an antigen and that the immune response can be enhanced by encapsulating the antigen and modifying the exterior of the encapsulin. Given their larger size, the uptake in macrophages can be increased compared with non-encapsulated proteins, ${ }^{33}$ which we also explore in Chapter 5. 
Even though an increased immune response can be advantageous when utilizing encapsulins as vaccine carriers, it is disadvantageous when used as protein drug carriers. While we further explore this principle in Chapter 5, Sonotaki et al. studied the possibility of PEGylating the encapsulin surface. ${ }^{34}$ They demonstrated that encapsulins can be PEGylated by binding polyethylene glycol (PEG) to the exterior of encapsulins. These encapsulins were modified to contain a his-tag on the C-terminus and the encapsulin structure itself was not compromised. PEGylation can reduce immunogenicity and increase bioavailability of proteins.

In this thesis, other modifications and utilizations of encapsulins, specifically B. linens and T. maritima, are described, showing the potential that encapsulins have for various other usages. The work described in this Chapter demonstrates that, while encapsulins are novel, there is a wide range of potential applications for which encapsulins can be used.

\subsection{Acknowledgements}

This chapter has its origin in the review article that I wrote together with dr. M. V. de Ruiter, dr. D. Luque, Prof. J. R. Castón and Prof. J. J. L. M. Cornelissen, all of whom I thank very kindly for their contributions. 


\subsection{References}

1. Andrews, S. C. The Ferritin-like Superfamily: Evolution of the Biological Iron Storeman from a Rubrerythrin-like Ancestor. Biochemica et Biophysica Acta - General Subjects. 2010, 1800, 691-705

2. Yeates, T. O.; Crowley, C. S.; Tanaka, S. Bacterial Microcompartments Organelles: Protein Shell Structure and Evolution. Annu. Rev. Biophys. 2010, 39, 185-205

3. Ladenstein, R.; Fischer, M.; Bacher, A. The Lumazine Synthase / Riboflavin Synthase Complex: Shapes and Functions of a Highly Variable Enzyme System. FEBS J. 2013, 280, 2537-2563

4. Valdes-Stauber, N.; Scherer, S. Isolation and Characterization of Linocin M18, a Bacteriocin Produced by Brevibacterium linens. Applied and Environmental Microbiology. 1994, 60, 3809-3814

5. Hicks, P. M.; Rinker, R. D.; Baker, J. R.; Kelly, R. M. Homomultimeric Protease in the Hyperthermophilic Bacterium Thermotoga maritima has Structural and Amino Acid Sequence Homology to Bacteriocins in Mesophilic Bacteria. FEBS Letters. 1998, 440, 393-398

6. Sutter, M.; Boehringer, D.; Gutmann, S.; Günther, S.; Prangishvili, D.; Loessner, M. J.; Stetter, K. O.; Weber-Ban, E.; Ban, N. Structural Basis of Enzyme Encapsulation into a Bacterial Nanocompartment. Nat, Struct. Mol. Biol. 2008, 15, 939-947

7. Giessen, T. W. Encapsulins: Microbial Nanocompartments with Applications in Bio-medicine, Nanobiotechnology and Materials Science. Curr. Opin. in Chem. Biol. 2016, 34, 1-10

8. Rahmanpour, R.; Bugg, T. D. Assembly in Vitro of Rhodococcus jostii RHA 1 Encapsulin and Peroxidase DypB to Form a Nanocompartment. FEBS. 2013, 280, 2097-2104 
9. Putri, R. M.; Allende-Ballestero, C.; Luque, D.; Klem, R.; Rousou, K. A.; Liu, A.; Traulsen, C. H.; Rurup, W. F.; Koay, M. S. T.; Castón, J. R.; Cornelissen, J. J. L. M. Structural Characterization of Native and Modified Encapsulins as Nanoplatforms for in Vitro Catalysis and Cellular Uptake. ACS Nano 2017, 11, 12796-12804

10. Williams, E. M.; Jung, S. M.; Coffman, J. L.; Lutz, S. Pore Engineering for Enhanced Mass Transport in Encapsulin Nanocompartments. ACS Synth. Biol. 2018, 7, 2514-2517

11. Snijder, J.; Kononova, O.; Barbu, I. M.; Uetrecht, C.; Rurup, W. F.; Burnley, R. J.; Koay, M. S.; Cornelissen, J. J.; Roos, W. H.; Barsegov, V.; Wuite, G. J.; Heck, A. J. Assembly and Mechanical Properties of the Cargo-Free and Cargo-Loaded Bacterial Nanocompartment Encapsulin. Biomacromolecules 2016, 17, 2522-2529

12. Huber, R.; Langworthy, T. A.; König, H.; Thomm, M.; Woese, C. R.; Sleytr, U. B.; Stetter, K. O. Thermotoga maritima sp. nov. Represents a New Genus of Unique Extremely Thermophilic Eubacteria Growing up to $90^{\circ} \mathrm{C}$. Arch. of Microbiol. 1986, 144, 324-333

13. Giessen, T. W.; Silver, P. A. Widespread Distribution of Encapsulin Nanocompartments Reveals Functional Diversity. Nat. Microbiol. 2017, 2, 17029

14. Künzle, M.; Mangler, J.; Lach, M.; Beck, T. Peptide-directed Encapsulation of Inorganic Nanoparticles into Protein Containers. Nanoscale. 2018, 10, 22917-22926

15. Nichols, R. J.; Cassidy-Amstutz, C.; Chaijarasphong, T.; Savage, D. F. Encapsulins: Molecular Biology of the Shell. Crit. Rev. in Biochem. and Mol. Biol. 2017, 52, 583-594

16. Cassidy-Amstutz, C.; Oltrogge, L.; Going, C. C.; Lee, A.; Teng, P.; Quintanilla, D.; East-Seletsky, A.; Williams, E. R.; Savage, D. F. Identification of a Minimal Peptide Tag for in Vivo Loading of Encapsulin. Biochemistry 2016, 55, 3461-3468 
17. McHugh, C. A.; Fontana, J.; Nemecek, D.; Cheng, N.; Aksyuk, A. A.; Heymann, J. B.; Winkler, D. C.; Lam, A. S.; Wall, J. S.; Steven, A. C.; Hoiczyk, E. A Virus Capsid-like Nanocompartment that Stores Iron and Protects Bacteria from Oxidative Stress. EMBO J. 2014, 33, 1896-1911

18. Giessen, T. W.; Orlando, B. J.; Verdegaal, A. A.; Chambers, M. G.; Gardener, J.; Bell, D. C.; Birrane, G.; Liao, M.; Silver, P. A. Large Protein Organelles Form a New Iron Sequestration System with High Storage Capacity. eLIFE. 2019, 8, e46070

19. Contreras, H.; Joens, M. S.; McMath, L. M.; Le, V. P.; Tullius, M. V.; Kimmey, J. M.; Bionghi, N.; Horwitz, M. A.; Fitzpatrick, J. A.; Goulding, C. W. Characterization of a Mycobacterium tuberculosis Nanocompartment and its Potential Cargo Proteins. J. Biol. Chem. 2014, 289, 18279-18289

20. Rurup, W. F.; Snijder, J.; Koay, M .S. T.; Heck, A. J.; Cornelissen, J. J. Self-sorting of Foreign Proteins in a Bacterial Microcompartment. J. Am. Chem. Soc. 2014, 136, 3828-3832

21. Lau, Y. H.; Giessen, T. W.; Altenburg, W. J.; Silver, P. A. Prokaryotic Nanocompartments form Synthetic Organelles in a Eukaryote. Nat. Comm. 2018, 9, 1311

22. Sigmund, F.; Massner, C.; Erdmann, P.; Stelzl, A.; Rolbienski, H.; Desai, M.; Bricault, S.; Wörner, T. P.; Snijder, J.; Geerlof, A.; Fuchs, H.; Hrabě de Angelis, M.; Heck, A. J. R.; Jasanoff, A.; Ntziachristos, V.; Plitzko, J.; Westmeyer, G. G. Bacterial Encapsulins as Orthogonal Compartments for Mammalian Cell Engineering. Nat. Commun. 2018, 9, 1990 
23. Tamura, A.; Fukutani, Y.; Takami, T.; Fujii, M.; Nakaguchi, Y.; Murakami, Y.; Noguchi, K.; Yohda, M.; Odaka, M. Packaging Guest Proteins into the Encapsulin Nanocompartment from Rhodococcus erythropolis N771. Biotechnol. Bioeng. 2015, 112, 13-20

24. Giessen, T. W.; Orlando, B. J.; Verdegaal, A. A.; Chambers, M. G.; Gardener, J.; Bell, D. C.; Birrane, G.; Liao, M.; Silver, P. A. Structure and Function of a 9.6 Megadalton Bacterial Iron Storage Compartment. bioRxiv. 2019

25. He, D.; Hughes, S.; Vanden-Hehir, S.; Georgiev, A.; Altenbach, K.; Tarrant, E.; Mackay, C. L.; Waldron, K. J.; Clarke, D. J.; MarlesWright, J. Structural Characterization of Encapsulated Ferritin Provides Insight into Iron Storage in Bacterial Nanocompartments. Elife. 2016, e18972

26. Sigmund, F.; Pettinger, S.; Kube, M.; Schneider, F.; Schifferer, M.; Schneider, S.; Efremova, M. V.; Pujol-Martí, J.; Aichler, M.; Walch, A.; Misgeld, T.; Dietz, H.; Westmeyer, G. G. Iron-Sequestering Nanocompartments as Multiplexed Electron Microscopy Gene Reporters. ACS Nano. 2019

27. Giessen, T. W.; Silver, P. A.; Converting a Natural Protein Compartment into a Nanofactory for the Size-Constrained Synthesis of Antimicrobial Silver Nanoparticles. ACS Synth. Biol. 2016, 5, 1497-1504 28. Moon, H.; Lee, J.; Min, J.; Kang, S. Developing Genetically Engineered Encapsulin Protein Cage Nanoparticles as a Targeted Delivery Nanoplatform. Biomacromolecules. 2014, 15, 3794-3801

29. Moon, H.; Lee, j.; Kim, H.; Heo, S.; Min, J.; Kang, S. Genetically Engineering Encapsulin Protein Cage Nanoparticles as a SCC-7 Cell Targeting Optical Nanoprobe. Biomater. Res. 2014, 18:21

30. Bae, Y.; Kim, G. J.; Kim, H.; Park, S. G.; Jung, H. S.; Kang, S. Engineering Tunable Dual Functional Protein Cage Nanoparticles Using Bacterial Superglue. Biomacromolecules. 2018, 19, 2896-2904 
31. Choi, B.; Moon, H.; Hong, S. J.; Shin, C.; Do, Y.; Ryu, S.; Kang, S. Effective Delivery of Antigen-Encapsulin Nanoparticle Fusions to Dendritic Cells Leads to Antigen-Specific Cytotoxic T Cell Activation and Tumor Rejection. ACS Nano. 2016, 10, 7339-7350

32. Lagoutte, P.; Mignon, C.; Stadthagen, G.; Potisopon, S.; Donnat, S.; Mast, J.; Lugari, A.; Werle, B. Simultaneous Surface Display and Cargo Loading of Encapsulin Nanocompartments and Their Use for Rational Vaccine Design. Vaccine. 2018, 36, 3622-3628

33. Pacheco, P.; White, D.; Sulchek, T. Effects of Microparticle Size and Fc Density on Macrophage Phagocytosis. PloS one 2013, 8, e60989 34. Sonotaki, S.; Takami, T.; Noguchi, K.; Odaka, M.; Yohda, M.; Murakami, Y. Successful PEGylation of Hollow Encapsulin Nanoparticles from Rhodococcus erythropolis N771 Without Affecting their Disassembly and Reassembly Properties. Biomat. Science. 2017, 5, 1082-1089 
Chapter 2 


\section{Chapter 3}

\section{Cryo-EM Analysis of the Structure of Encapsulins}

\section{Abstract}

While the molecular structure of some encapsulins, such as Thermotoga maritima encapsulin, have been established, additional exploration of other encapsulin structures will increase our understanding of, and ability to modify encapsulins. The genetic similarity between encapsulins has been determined, but the impact of the small differences on the encapsulin structure remains unknown. We address this deficiency by exploring the native structure of Brevibacterium linens encapsulin with dye decolorizing peroxidase cargo. We compare this with the T. maritima encapsulin structure, from which a crystal structure is available, which privodes more insight in the uniformity and origin of encapsulins.

Allende-Ballestero, C.; Klem, R.; Luque, D.; Cornelissen, J. J. L. M.; Castón, J. R. Cryo-EM Analysis of the Structure of Encapsulins. Manuscript in preparation. 


\subsection{Introduction}

Encapsulins are icosahedral protein nanoparticles ranging in size from $20-40 \mathrm{~nm}$. The encapsulin atomic structure from Thermotoga maritima (TmE), was resolved in 2008 by Sutter et al. ${ }^{1}$ Structures from encapsulins derived from other bacteria have been determined since $^{2,3}$ and detailed analysis of parts of the structure has been performed, such as the pores. ${ }^{4}$ They generally conform to a $\mathrm{T}=1$ or $\mathrm{T}=3$ structure and consist of 60 or 180 monomers, respectively. The structures show high similarity, both in the complex structural level and at the monomer level, as shown with the Mycococcus xanthus encapsulin (MxE) by McHugh et al. ${ }^{5}$

Imaging encapsulins at high resolution and determining the atomic structure provides structural insight and makes specific, targeted modification of the encapsulins easier and can provide more insight into the origins of encapsulins. In addition, detailed structural knowledge of encapsulins can also increase the variety of cargo proteins, as the interaction between the affinity tag that is present on most encapsulin cargo proteins and the encapsulin shell itself is poorly understood. ${ }^{1,6}$ Putri et al. provided insight in the differences in the structure of B. linens encapsulin (BlE) that contained different cargos. ${ }^{3}$ They used monomeric teal fluorescent protein (mTFP) as a non-native cargo. In this study we focus on the BlE structure when loaded with its native cargo (Dye decolorizing peroxidase $(\mathrm{DyP}))$.

While the cargo of encapsulins has thus far been modified to contain protein dyes (such as GFP, mNeonGreen ${ }^{7}$ and monomeric teal fluorescent protein $(\mathrm{mTFP}))^{3}$ and enzymes (such as tyrosinase and others), 8, 9 encapsulation of a medically relevant enzyme has not been done. We use L-asparaginase (ASNase) as protein cargo in Chapter 4, here we study the binding of the DyP to the encapsulin shell. An increased understanding of this interaction could help with correctly loading non-native enzymes in 
encapsulins and help determining the maximum volume of cargo that can be loaded. The correct structure and folding is essential when enzymes are encapsulated, as they could otherwise have reduced or no activity.

In this chapter, we use cryo electron microscopy (cryo-EM) to analyze the structure of BlE loaded with its native cargo DyP (BlE-DyP). We also study how this structure compares with the crystal structure of Thermotoga maritima encapsulins, as determined by Sutter et al. in $2008,{ }^{1}$ and with other structures that have have demonstrated genetic or structural similarity with encapsulins. This can provide more insight in how the structure of the encapsulins relate to their function.

\subsection{Results and Discussion}

\subsubsection{Production of BlE-DyP}

To study the structural differences of encapsulins between various bacterial origins, we used encapsulins derived from BlE from our lab and the crystal structure from TmE as determined by Sutter et al. ${ }^{1}$ To study the cargo interaction with the encapsulin shell, we used the BlE with its native cargo, BlE-DyP.

After purification we analyzed the encapsulins using fast protein liquid chromatography (FPLC), dynamic light scattering (DLS) and transmission electron microscopy (TEM) (Figure 3.1A, B and C, respectively). These data suggest the correct folding of encapsulin into $\sim 20 \mathrm{~nm}$ sized particles. 


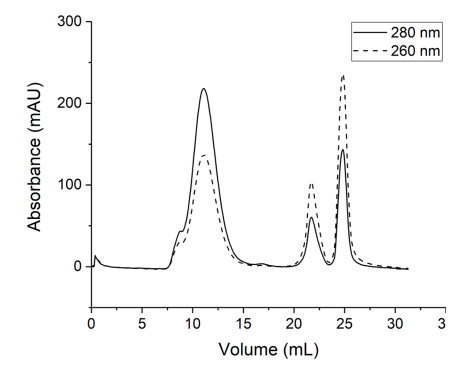

(a)

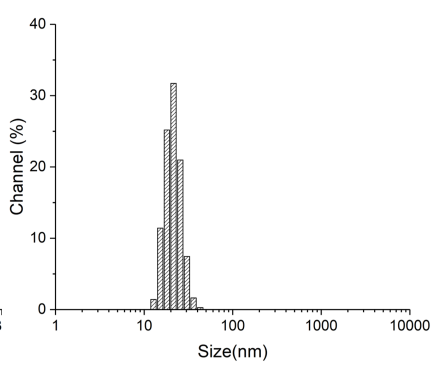

(b)

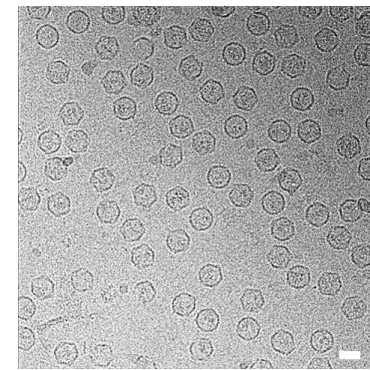

(c)

Figure 3.1: Characterization of the DyP-filled BlE: SEC (A), DLS (B) and TEM (C). The scale bar in C represents $20 \mathrm{~nm}$. With SEC, we isolated the elution peak at $11.5 \mathrm{~mL}$.

\subsubsection{Production of BlE-ASNase}

We also made a modified cargo by genetically removing the DyP sequence and replacing it with type II ASNase. This was sequenced by Eurofins and using the samples with the correct sequences we produced the encapsulins in the same way as the BlE-DyP. Analyses of these BlE-ASNase are found in Chapter 4.

\subsubsection{Cryo-EM Structural Analysis of BlE-DyP}

We used cryo-EM to study the structure of BlE-DyP. While Putri et al. provided insight in the BlE structure, ${ }^{3}$ we obtained higher resolution (2.65 $\AA)$ images that provide more detailed information on the BlE structure (Figure 3.2). The exterior diameter of $\mathrm{BlE}$ is $23 \mathrm{~nm}$ and the structure is icosahedral, with two-, three- and five-fold symmetry axes (Figure 3.2A$\mathrm{C}$, respectively). The interior is $18.5 \mathrm{~nm}$, meaning that the thickness of the shell is $4.5 \mathrm{~nm}$. The main pores in this shell are located at the threeand five-fold axes (Figure 3.2B and C). Additionally, we found a crevice on both sides of the two-fold axis (Figure 3.2A). 

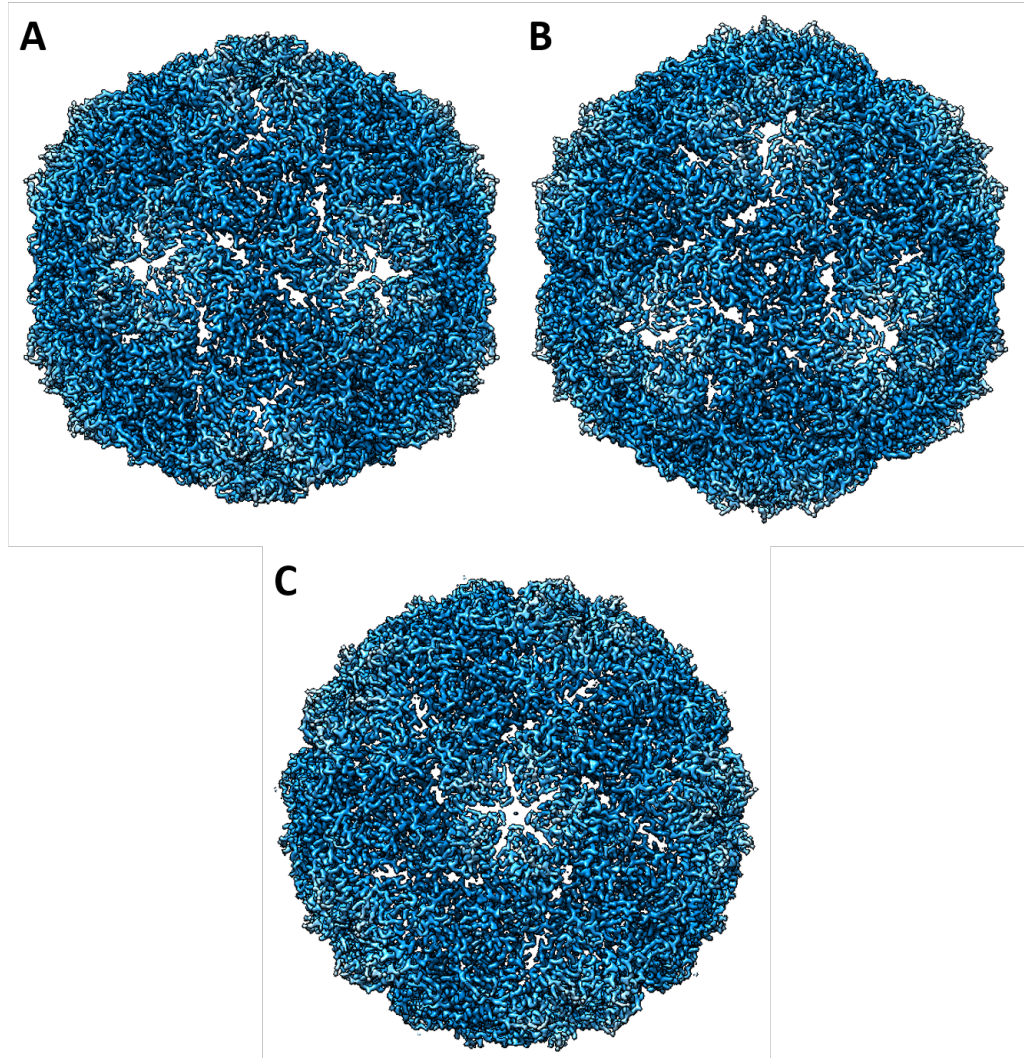

Figure 3.2: 3D reconstruction of BlE-DyP as seen centered on the two-fold (A), three-fold (B) and five-fold (C) axis.

The pores at the three-fold axes are $7 \AA$ in diameter and the five-fold axis are $14 \AA$ in size, with the crevices having a length of $31 \AA$. This pore size at the five-fold axis is larger than the size found by Rahmanpour et al. in the Rhodococcus jostii RHA1 encapsulin $(\mathrm{RjE}),{ }^{10}$ who also suggested that these pores are dynamic. We studied this dynamicity by analyzing the flexibility of peptides in the encapsulin shell using the differences in resolution as determined by cryo-EM; if a structure is very flexible, it will result in a lower resolution. The structure of BlE shows little flexibility at most of the encapsulin exterior except for the five-fold axes, where the resolution is lower (Figure 3.3A). 
On the interior, we found higher incidents of flexibility, but the main flexible points are also located at the five-fold axes (Figure 3.3B). This supports the hypothesis proposed by Rahmanpour et al. that the pores are dynamic. ${ }^{10}$ The extent of this dynamicity requires additional study. It does suggest that, to a degree, size is not the determining factor for which molecules can be transported across the protein shell. The influence of other effects such as hydrophobicity and charge remain unknown.

A

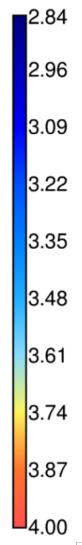

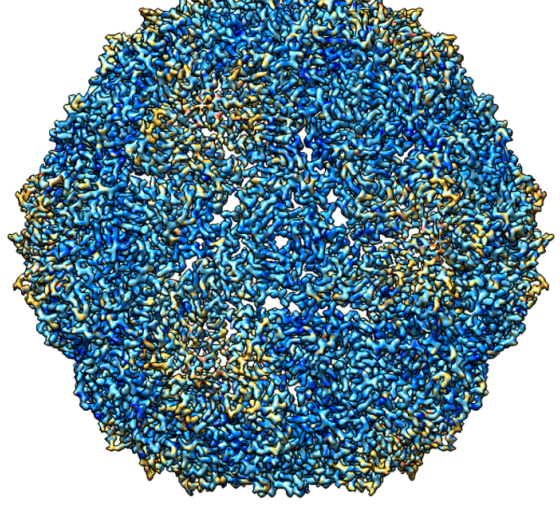

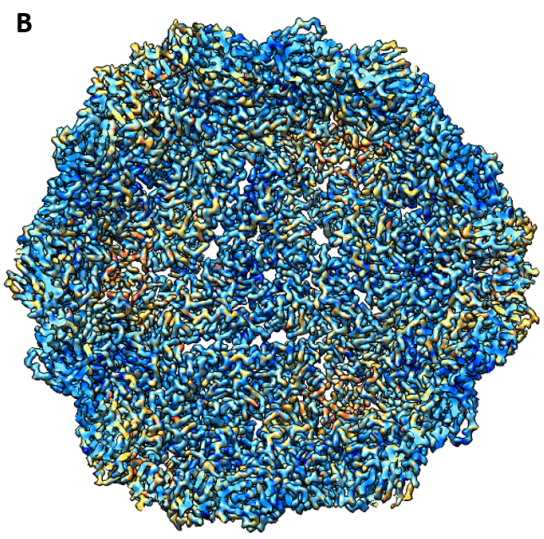

Figure 3.3: Local resolution of BlE-DyP on the exterior (A) and interior (B). Blue highlights increased local resolution while red highlights decreased local resolution.

Surrounding the pore at the five-fold axis of BlE are 5 loops, each consisting of a TDHGYP amino acid sequence. When analyzing this sequence with the online program ExPASy (ProtParam), we found that the pores are negatively charged and hydrophilic (hydropathicity $=-1.783$ ). While the exact nature of the pores requires further investigation, this does suggest that negatively charged or hydrophobic molecules are less likely to pass through the pores. 
The structure of BlE with a non-native cargo, ASNase, is not significantly different from the structure of native encapsulin; BlE-DyP (see Chapter 4, Figure 4.4). This suggests that the cargo of BlE does not affect the BlE structure, although the lack of cargo could still influence the structure, as was suggested by Putri et al and Snijder et al. ${ }^{3,11}$

Using cryo-EM, we determined the atomic structure of the BlE monomers and we extrapolated the whole structure. These monomers interlock with each other forming the icosahedral encapsulin structure, from which dimers (Figure 3.4A and B), trimers (Figure 3.4C and D) and pentamers (Figure $3.4 \mathrm{E}$ and $\mathrm{F}$ ) can be discriminated. The E-loop connecting the dimers is the main linker between trimers and couples multiple pentamers in BlE. These multimers have been studied before, with suggestions that encapsulins consist of pentamer or dimer complexes. ${ }^{12}$ We found that the trimer organization is also present in the encapsulin structure. The trimers show high interlocking with other trimers (Figure 3.4D), where the E-loop again forms the bridge between trimers, which could explain the high stability of encapsulins. In BlE and TmE, the dimers are likely the main building block. 
A

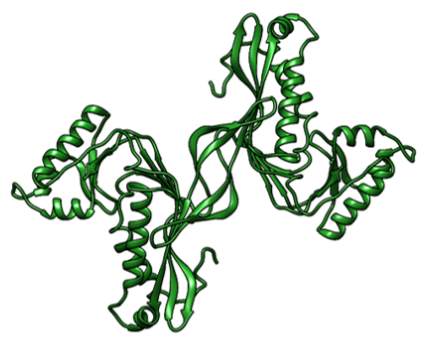

C

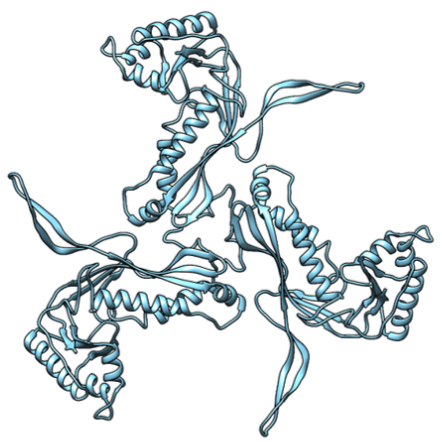

$\mathbf{E}$

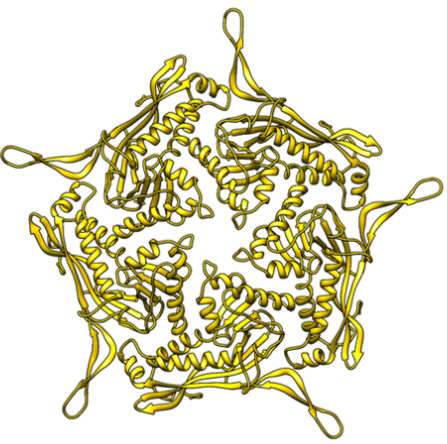

B

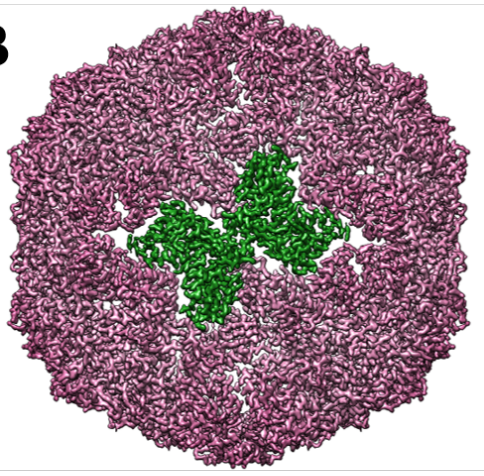

D

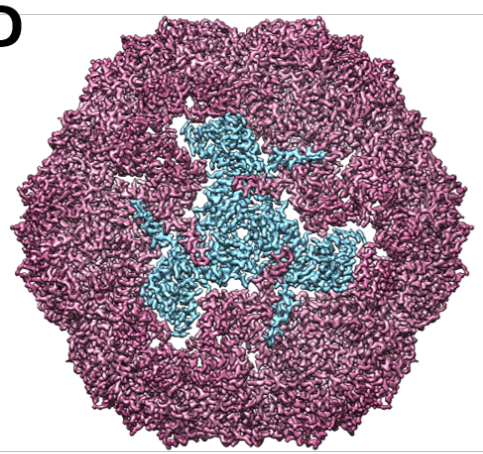

$\mathbf{F}$

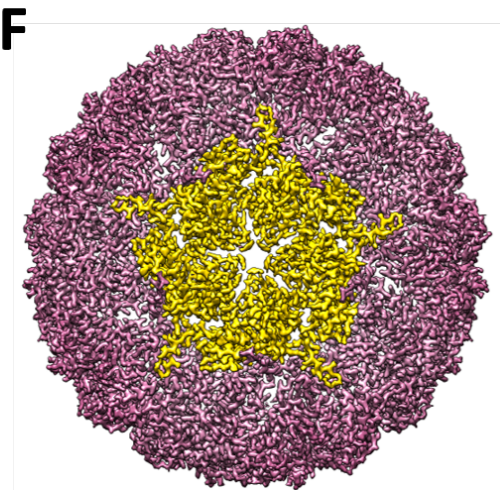

Figure 3.4: Atomic structure of BlE DyP dimer (A), which is highlighted in the complete BlE structure in (B). The orientation is centered on the two-fold axis. The trimer $(\mathrm{C})$ and the trimer in the complete BlE structure (D) are centered on the three-fold axis, while the pentamer (E) and the pentamer in the complete BlE structure $(\mathrm{F})$ are centered on the fivefold axis. 


\begin{tabular}{llll}
$\mathrm{T}=1$ & $\mathrm{~T}=\mathbf{3}$ & $\mathrm{T}=7$ & $\mathrm{~T}=7$ \\
\cline { 2 - 4 } $\mathrm{BIE}$ & $\mathrm{MXE}$ & HK97 & P22
\end{tabular}
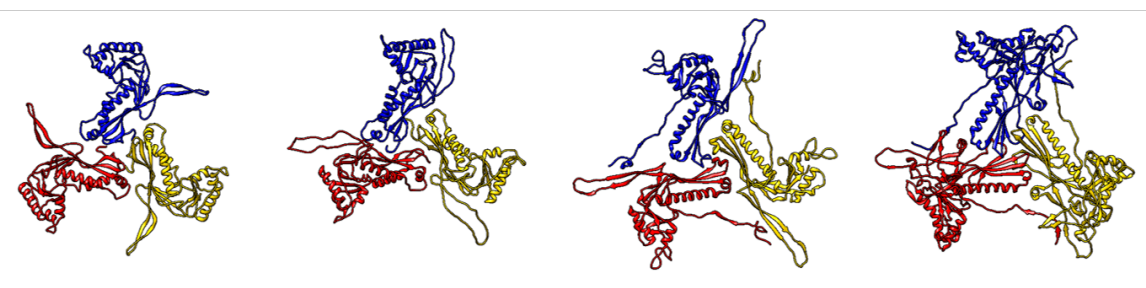

Figure 3.5: Similarity of trimers in BlE, MxE, the Hong Kong 97 Virus and the bacteriophage P22. The triangulation number of each assembled protein cage is profided above the trimers.

This trimeric organization is also seen in other encapsulins or encapsulinlike structures (Figure 3.5). Regardless of the triangulation number $(1,3$, or 7$)$, the trimeric organization is present. The difference in the trimeric structure originates from the differences in the monomeric structure. These monomeric structures, however, have a high similarity between different encapsulins. $\mathrm{BlE}$ and $\mathrm{TmE}$, that both conform to $\mathrm{T}=$ 1 structures, have high overlap of their monomer structures, while this overlap is lower between $\mathrm{BlE}$ and monomers of protein nanocages that conform to $\mathrm{T}=3$ or $\mathrm{T}=7$ (Figure 3.6 ). 


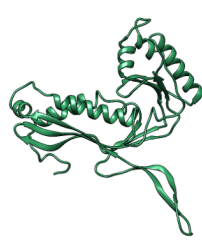

BIE

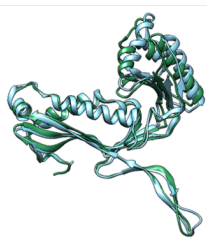

BIE vs. TmE

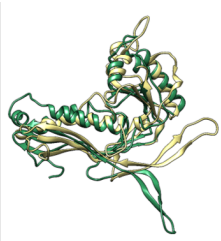

BIE vs. Prohead HK97

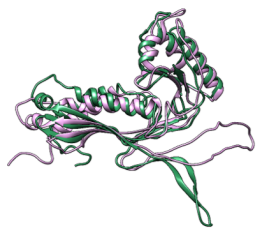

BIE vs. MxE

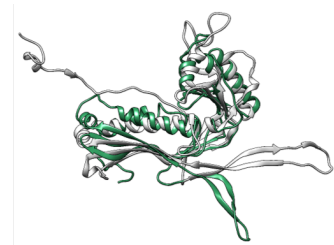

BIE vs. Head HK97

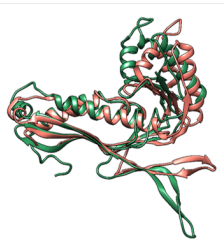

BIE vs. PfE

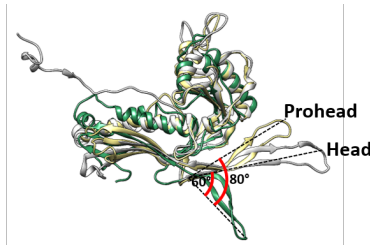

BIE vs. Prohead vs. Head HK97

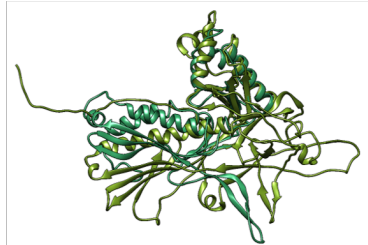

BIE vs. P22

Figure 3.6: Overlay of atomic structure of BlE monomer with monomers of other protein nanocages that have a similar structure. The BlE monomer is always in green. TmE is, like BlE, a $\mathrm{T}=1$ icosahedral structure, while the other monomers conform to $\mathrm{T}=3$ or $\mathrm{T}=7$ icosahedral structures. The monomers were derived from the Protein Data Bank with the following entries; Prohead HK 97: 3E8K, ${ }^{13}$ Head HK97: 2FT1, ${ }^{14}$ TmE: 3DKT, ${ }^{1} \mathrm{MxE}: 4 \mathrm{PT} 2^{5}$, PfE: 2E0Z, ${ }^{15}$ P22: $5 \mathrm{UU} 5 .{ }^{16}$

With these other encapsulins (MxE and PfE) and bacteriophages (HK97 head and prohead and $\mathrm{P} 22$ ), which conform to $\mathrm{T}=3$ or $\mathrm{T}=7$ structures, the main difference is the orientation of the E-loop (Figure 3.6). McHugh et al. showed that the structures with a $\mathrm{T}=3$ or $\mathrm{T}=7$ conformation have their E-loop oriented parallel to the bulk of the monomer, ${ }^{5}$ which is displayed in Figure 3.6, while the $\mathrm{T}=1 \mathrm{TmE}$ has its E-loop oriented outward, similar to BlE monomers (Figure 3.6). Since the main body (P and A-domains) are structurally conserved between all monomers, the E-loop orientation is probably the defining factor that determines the triangulation number of encapsulins. This is also visible in the trimeric (Figure 3.5) and pentameric (Figure 3.7) organization of the different encapsulins. 

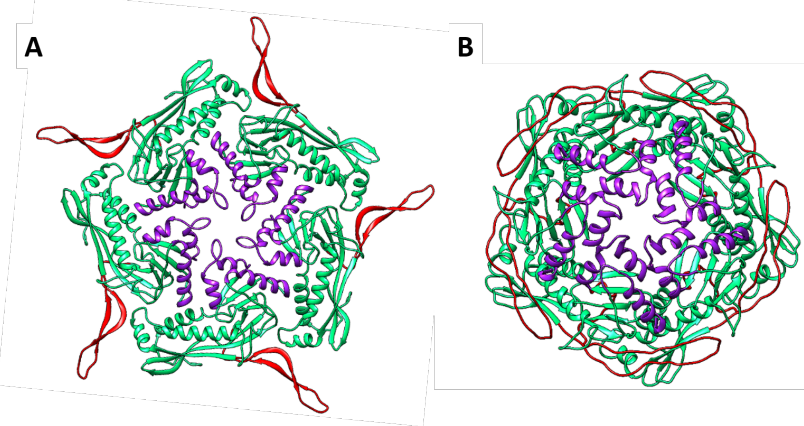

Figure 3.7: Pentamers of $\mathrm{BlE}(\mathrm{A})$ and $\mathrm{MxE}(\mathrm{B})$. The orientation of the E-loop (red) is the main difference between these pentamers. The MxE pentamer was derived from the Protein Data Bank entry 4 PT2. ${ }^{5}$

This suggests that the modification of this loop could allow the formation of $\mathrm{T}=3 \mathrm{BlE}$ particles, meaning that the size of the encapsulin can be modified. Potentially, given the similarity of the encapsulin monomers, new protein nanocages can be made by mixing monomers from different encapsulins, creating heteromultimeric encapsulins.

We found that the BlE structure is mainly negatively charged, with the exterior being predominantly negatively charged, while the interior has negatively charged patches (Figure 3.8A and B). Künzle et al. used these patches in TmE to encapsulate positively charged gold nanoparticles. ${ }^{6}$
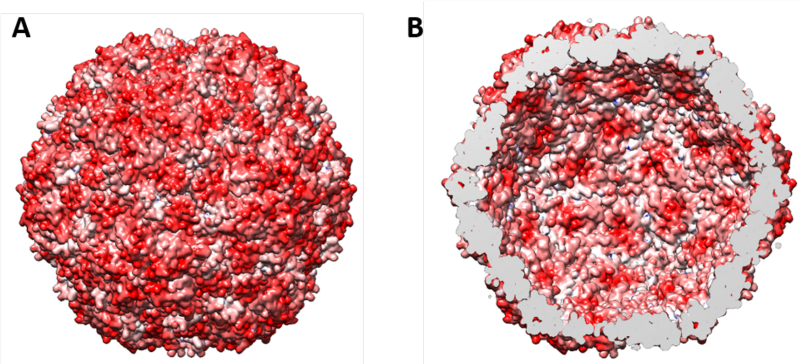

Figure 3.8: Charge distribution of BlE exterior (A) and interior (B) with red corresponding to negative charge. 


\subsubsection{Analysis of the Affinity Tag Binding Site}

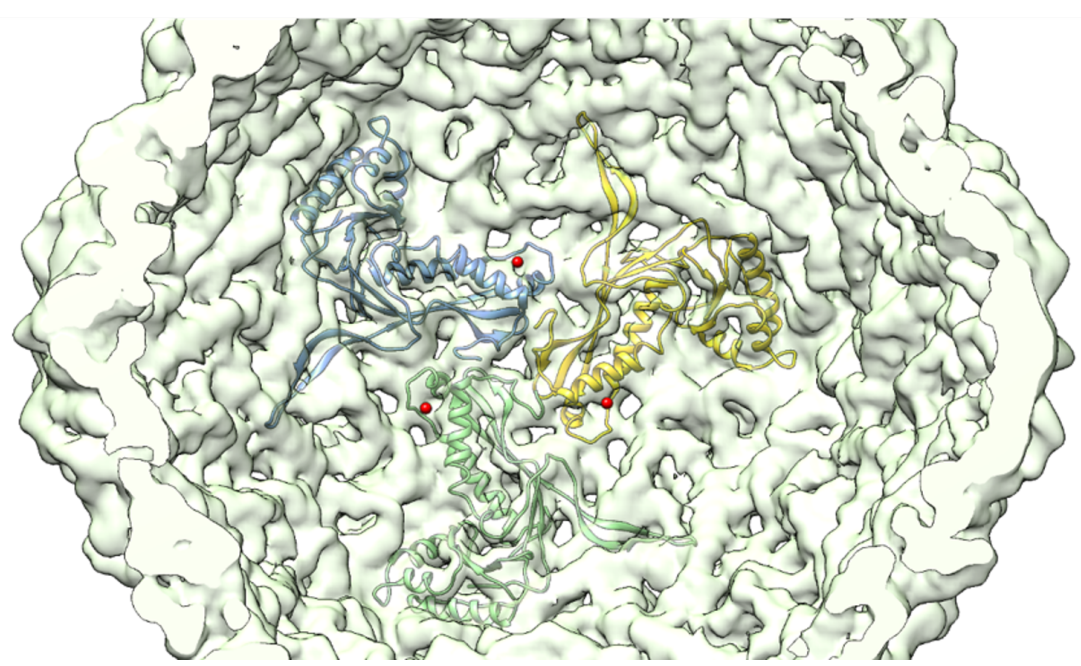

Figure 3.9: A 3D model of $\mathrm{BlE}$ with three monomers highlighted that are involved in the binding of the DyP cargo. The binding sites are highlighted with red dots.

The cargo in encapsulins interacts with the shell using an affinity tag. This affinity tag on the cargo of different encapsulins is different, but hydrophobic residues are highly conserved. ${ }^{17}$ This suggests that the binding sites of the encapsulins also have hydrophobic residues to provide hydrophobic interactions. The binding sites for the DyP cargo are located at the A-domains of the encapsulin monomers, in a clear triangular shape (Figure 3.9). The triangular structure of the binding highlights the trimers in the structural organization.

We overlayed this binding site from BlE with the binding site from TmE (Figure 3.10A). Künzle et al. demonstrated the fitting of the affinity tag into this binding site, while we give a detailed structural analysis of this site. $^{6}$ 
A

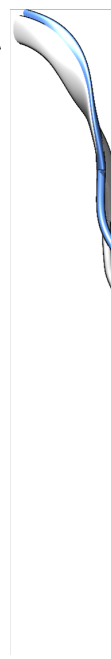

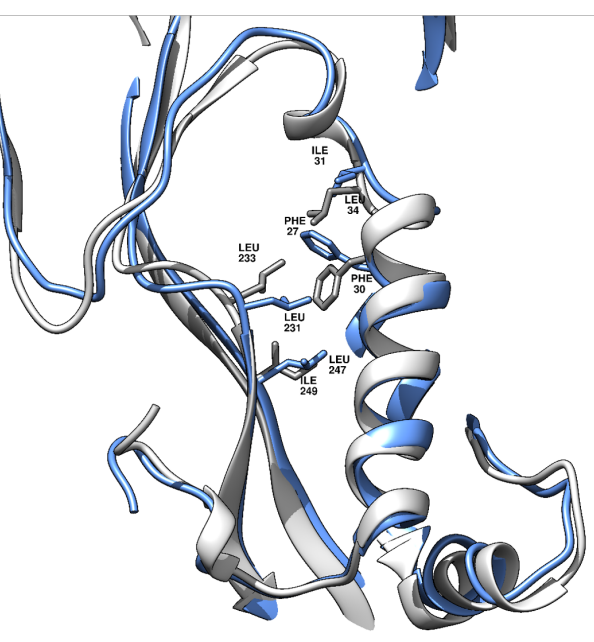

B

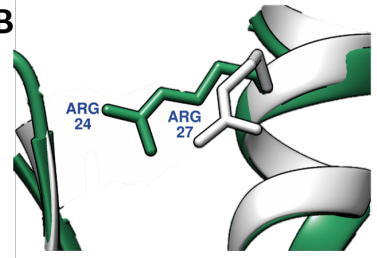

Figure 3.10: (A) shows the binding site in both BlE (blue) and TmE (white) and (B) demonstrates the arginine residue at the opening of the binding site.

We found that there is significant structural similarity between the binding sites of both $\mathrm{BlE}$ and TmE, with primarily hydrophobic residues being conserved in the genetic sequence. These hydrophobic residues are phenylalanine, two leucines and an isoleucine and they are found in both types of encapsulin (Figure 3.10A). This also suggests that indeed hydrophobic interactions stabilize the cargo within the encapsulin. The binding site appears to be closed by an arginine in the TmE sample while it is unblocked in the BlE sample when there was no cargo and hence the site is not used by the affinity tag (Figure 3.10B). Given that permanent closure of the binding pocket might prevent the affinity tag from binding, the "open" and "closed" conformations are probably occurring in both TmE and BlE and are probably dependent on whether or not the binding site is occupied. 


\subsubsection{Discussion}

Since the first structural characterization of encapsulins done by Sutter et al. the structure of encapsulins has slowly been revealed. ${ }^{1}$ In this chapter, we performed a detailed structural analysis of BlE using cryo-EM. This increased the structural understanding of encapsulins which in turn provides insight in potential applications.

The high density of the encapsulin shell was an established fact, but there are more pores than previously assumed. Pores are located at the five-, three- and two-fold axes, but the exact nature of the two-fold axis pores is not so much a pore as it is a crevice. We also found that the five-fold axis pore is dynamic in nature, which was already suggested by Rahmanpour et al. ${ }^{10}$ This implies that more molecules than initially assumed can potentially pass this encapsulin shell.

Additionally, we found that, at least for $\mathrm{T}=1 \mathrm{BlE}$ and $\mathrm{TmE}$, that dimers are the leading building blocks for the encapsulins, which corresponds to results from Snijder et al. ${ }^{12}$ Furthermore, we found trimeric organization in BlE as well. The trimers are highly intertwined, which could explain the high stability recorded for encapsulins. These trimeric interactions could be related to the orientation of the E-loop of the monomers. When pointed outwards, like in BlE and TmE, the particles conform to a $\mathrm{T}=$ 1 icosahedral structure and have clear interactions between trimers that use the E-loop. The importance of the trimeric organization is further supported by the fact that the DyP cargo interacts in 3 places with the encapsulin shell.

However, when this E-loop is pointed parallel to the structure, like in the MxE, this does not occur. The E-loops do not intertwine and this suggests that a dimeric build-up of the particle is less stable as the interaction between dimers is weaker than in $\mathrm{BlE}$ and $\mathrm{TmE}$. The parallel 
nature of the E-loop results in two principles; it prevents the E-loop linking to the $\mathrm{P}$-domain because it is not pointing outwards and it blocks the linking site for other E-loops. We suggest that in encapsulins the E-loop orientation is critical in the formation of the highly stable $\mathrm{T}=1$ icosahedral nanoparticles. This could be verified by modifying the E-loop of $\mathrm{BlE}$ to be parallel to the rest of the monomer. If this results in a $\mathrm{T}=3$ or $\mathrm{T}=7$ encapsulin, like MxE or HK97, then it confirms that the E-loop orientation is the main factor determining the encapsulin structure.

We found high structural similarity between TmE and BlE. Given that the genetic sequence is also highly conserved, it is probable that the information obtained for BlE can also be applied to other $\mathrm{T}=1$ encapsulins.

\subsection{Conclusions}

The structure of encapsulins is relatively dense, but is more porous than previously assumed. At the two-, three- and five-fold axes, pores and crevices are present. The pores at the five-fold axes are dynamic in nature. Additionally, the triangulation number of the encapsulins is probably determined by the orientation of the E-loop. The structural information provides more insight in the possibilities of encapsulin as a drug or enzyme carrier, especially regarding the transport of small molecules across the protein shell.

\subsection{Acknowledgements}

I thank dr. J. R. Castón, dr. D. Luque and M. C. Allende-Ballestero for the fruitful collaboration on this project and their fantastic work on the cryo-EM analyses. 


\subsection{Experimental Section}

\subsubsection{Materials}

All chemicals were purchased from Sigma-Aldrich unless stated otherwise. Buffers were prepared using double deionized water from a Millipore system.

\subsubsection{Genetic Modification of Plasmids with BlE- GFP to BIE-ASNase}

Using "A plasmid Editor" (ApE, version 2.0.55), a gene encoding for Type II ASNase was designed based on the genetic sequence derived from the Protein Data Bank. A plasmid encoding for this gene was produced by Eurofins, using a pEX-K4 plasmid. The 5' restriction site was XbaI and the 3' restriction site was XmaI. The genetic sequence was optimized for use in Escherichia coli (E. coli).

We transfected the plasmids into Nova Blue E. coli bacteria using the heat shock mechanism. $5.4 \mu \mathrm{g}$ plasmid was dissolved in $450 \mu \mathrm{L}$ MilliQ. The Nova Blue E. coli bacteria were taken from $20 \mu \mathrm{L}$ cryo stocks $\left(-80^{\circ} \mathrm{C}\right)$ and placed on ice. One $\mu \mathrm{L}$ of the plasmid sample was taken and added to the bacteria. This was put on ice for $5 \mathrm{~min}$, after which a heat shock (30 sec at $42{ }^{\circ} \mathrm{C}$ ) was applied, followed by 2 min on ice. Next, $80 \mu \mathrm{L}$ Super Optimal broth with Catabolite repression (SOC) medium was added. From this mixture, $50 \mu \mathrm{L}$ was placed on a shaker (MaxQ 4000, Thermo Fisher) for $1 \mathrm{~h}$ at $250 \mathrm{rpm}$. The $50 \mu \mathrm{L}$ samples were plated on agar plates supplemented with $0.1 \mathrm{mg} / \mathrm{mL}$ ampicillin (AMP) and 34 $\mu \mathrm{g} / \mathrm{mL}$ chloramphenicol (CAM) to culture bacteria colonies. The plates were placed in incubators at $37^{\circ} \mathrm{C}$ overnight. 
After incubation, colonies were taken with a sterile pipette tip and transferred to a culture tube containing $6 \mathrm{~mL}$ LB medium supplemented with $0.1 \mathrm{mg} / \mathrm{mL}$ AMP and $34 \mu \mathrm{g} / \mathrm{mL}$ CAM to create starter cultures. This was cultured at $37^{\circ} \mathrm{C}$ overnight in an incubator rotating at $250 \mathrm{rpm}$. The next day, cryo stocks were made using the culture tube content. For each cryo stock, $750 \mu \mathrm{L}$ sample was mixed with $250 \mu \mathrm{L} 60 \%$ (w/v) glycerol and transferred to $1 \mathrm{~mL}$ cryo-tubes.

The rest of the starter culture was used to extract the plasmids from. Thus, the QIAprep Spin Miniprep Kit (Qiagen) was used to extract the DNA from the bacteria, according to the protocol from the kit. After purification, the plasmids were stored at $4{ }^{\circ} \mathrm{C}$. The experiments following this preparation were done using the DNA samples that showed the highest concentration of DNA and had an absorbance $\lambda=260 / 280$ ratio closest to 1.8 , with 1.8 indicating a high purity of the DNA with regards to contaminations like RNA and proteins.

A starter culture was made with Rosetta gami DE3 E. coli that contained the plasmid encoding for BlE-GFP. This was done as described earlier in this chapter. From this starter culture, the DNA was extracted using the QIAprep Spin Miniprep Kit. As before, the DNA samples with the highest yield and purity (260/280 ratio) were taken. This DNA was also stored at $4{ }^{\circ} \mathrm{C}$.

Restriction digestion was performed using the stored DNA of the plasmids containing the genetic sequence coding for BlE-GFP, provided by dr. Rurup, and the plasmids containing the genetic sequence for ASNase. $1 \mu \mathrm{L}$ of XmaI (New England Biolabs), $1 \mu \mathrm{L}$ XbaI (New England Biolabs), $5 \mu \mathrm{L}$ cutsmart buffer (New England Biolabs) and $15 \mu \mathrm{L}$ of the respective DNA was mixed and with nuclease-free Milli-Q the volume was filled to $30 \mu \mathrm{L}$. This was put in a polymerase chain reaction (PCR (Primus 25 
Advanced Thermocycler, Peqlab)) with the following settings; $16 \mathrm{~h}$ at $37^{\circ} \mathrm{C}, 20$ min at $80^{\circ} \mathrm{C}$ and then stored at $4^{\circ} \mathrm{C}$.

To separate the plasmids from the cut gene, agarose gel electrophoresis was used. A $1 \%(\mathrm{w} / \mathrm{v})$ agarose gel was made (1 gram agarose dissolved in $100 \mathrm{~mL}$ nuclease-free Milli-Q, heated by microwave) with 10 $\mu \mathrm{L}$ SYBR safe ((Z)-4-((3-Methylbenzo[d]thiazol-2(3H)-ylidene)methyl)-1propylquinolin-1-ium 4-methylbenzenesulfonate (Thermo Fisher)) to visualize the DNA bands. The gel was run at $100 \mathrm{~V}$ until the bands were clearly separated. Afterwards, the bands corresponding to the plasmids containing the ASNase gene and the bands corresponding to the plasmids that contained the encapsulin gene without the GFP cargo, were recovered using the Wizard ${ }^{\circledR}$ SV Gel and PCR Clean-Up System Kit (Promega). The gels were cut out and dissolved in membrane binding buffer using $1 \mu \mathrm{L}$ per $1 \mathrm{mg}$ gel. Further, the protocol that was provided with the Wizard ${ }^{\circledR}$ Kit was followed.

After verification with the Nanodrop system (NanoDrop 1000 Spectrophotometer (Thermo Fisher)) to prove the presence of DNA, the samples that contained sufficient amounts of DNA were used for ligation. The ligation was performed by first mixing: $2 \mu \mathrm{L}$ T4 DNA ligase buffer; 7.5 $\mu \mathrm{L}$ plasmids; $8.5 \mu \mathrm{L}$ nuclease-free Milli-Q and $1 \mu \mathrm{L}$ T4 DNA ligase. This mixture was incubated for $2 \mathrm{~h}$ at room temperature. Next, the plasmids, which should now contain the operon coding for BlE and ASNase, were send to Eurofins for sequencing; this verified that the genetic modification was performed correctly. The correct samples were transfected to Nova Blue E. coli according to the heat shock mechanism described previously. These bacteria were plated on agar plates and incubated overnight at $37^{\circ} \mathrm{C}$. 
After the incubation, starter cultures were made, as described previously, to increase the amount of plasmids. The plasmids were extracted again using the QIAprep Spin Miniprep Kit and the plasmids were transfected to Rosetta gami DE3 E. coli, again using the heat shock mechanism described before. Another starter culture was made with these Rosetta gami DE3 E. coli bacteria, from which a cryo stock was made according to procedures described earlier. These were stored at $-80^{\circ} \mathrm{C}$.

\subsubsection{Production of BlE}

Using the cryo stock from the previous paragraph, new starter cultures were made as described earlier in this chapter. After overnight incubation at $37^{\circ} \mathrm{C}$ at $250 \mathrm{rpm}$, the samples were transferred to a $2 \mathrm{~L}$ Erlenmeyer which contained $500 \mathrm{~mL}$ LB medium $(20 \mathrm{~g}$ LB broth dissolved in $1 \mathrm{~L}$ Milli-Q, autoclaved) supplemented with $0.1 \mathrm{mg} / \mathrm{mL}$ AMP and $34 \mu \mathrm{g} / \mathrm{mL}$ CAM. These were placed at $37^{\circ} \mathrm{C}$ at $200 \mathrm{rpm}$ and the optical density at $600 \mathrm{~nm}\left(\mathrm{OD}_{600}\right)$ was checked. When it reached a value between 0.6 0.8, $50 \mu \mathrm{L}$ isopropyl $\beta$-D-1-thiogalactopyranoside (IPTG) was added (final concentration $0.1 \mathrm{mM}$ ) to stimulate gene expression. The protein production was kept overnight at $22^{\circ} \mathrm{C}$ and $200 \mathrm{rpm}$. Next, the samples were centrifuged at $8,500 \mathrm{rpm}, 10^{\circ} \mathrm{C}, 15 \mathrm{~min}$ using a Allegra ${ }^{\mathrm{TM}} 25 \mathrm{R}$ Centrifuge (Beckman Coulter) with a fixed angle TA-10-250 rotor (Beckman Coulter). The pellets were stored at $-20^{\circ} \mathrm{C}$ until further use.

Afterwards, the pellets were dissolved in $10 \mathrm{~mL}$ "encapsulin buffer" (20 $\mathrm{mM}$ Trizma Base, $150 \mathrm{mM} \mathrm{NH}_{4} \mathrm{Cl}, 20 \mathrm{mM} \mathrm{MgCl}_{2}$, pH set to 7.5 with $\mathrm{HCl}$ ). Using a tip sonicator (Branson Sonifier 250, 3 min, 50\% duty cycle, power 3), the bacteria were disrupted. Ten $\mu \mathrm{L}$ DNAse (Thermo Fisher) was added and this was incubated for $2 \mathrm{~h}$ at room temperature. Subsequently, the samples were ultracentrifuged at $40,000 \mathrm{rpm}, 10^{\circ} \mathrm{C}$, for 15 min using a Sorvall WX 80 Ultra Series Centrifuge (Thermo Fisher) with a fixed angle T-865 rotor (Thermo Fisher). 
The supernatant containing the encapsulins was recovered from the centrifuge tubes and to each $10 \mu \mathrm{L}$ RNAse (from bovine pancreas, Thermo Fisher) was added. This was incubated for $2 \mathrm{~h}$ at room temperature, after which the samples were placed on $10 \mathrm{~mL} 38 \%$ (w/v) sucrose. This was ultracentrifuged at $40,000 \mathrm{rpm}, 10^{\circ} \mathrm{C}$, for $17 \mathrm{~h}$. The bottom $3 \mathrm{~mL}$ were recovered and concentrated using Amicon spin filters (100 kDa Molecular Weight Cut-Off (MWCO)) and centrifuging at 3,000 rpm at $10^{\circ} \mathrm{C}$ with a Heraeus Multifuge X3R centrifuge and a 75003607 rotor (both Thermo Fisher) until there was $1 \mathrm{~mL}$ of the samples left.

Meanwhile, 10 - 50\% continuous sucrose gradients were prepared by gradually mixing $10 \%(\mathrm{w} / \mathrm{v})$ sucrose with $50 \%(\mathrm{w} / \mathrm{v})$ sucrose and transporting it to centrifuge tubes (Sorvall X25 (Thermo Fisher)) using a peristaltic pump (2232 Microperpex, LBK Bromma). The $1 \mathrm{~mL}$ samples were placed on top of the gradient and this was ultracentrifuged at $28,000 \mathrm{rpm}, 10^{\circ} \mathrm{C}$, for 17.5 h using swing-out buckets (Surespin 630-36 (Thermo Fisher)). After ultracentrifugation, the top $20 \mathrm{~mL}$ was removed and the next $9 \mathrm{~mL}$ were recovered.

This fraction was placed in 12 - $14 \mathrm{kDa}$ dialysis bags (Spectra/Por ${ }^{\circledR}$ 4 Dialysis Membrane (Spectrumlabs)) and placed in $800 \mathrm{~mL}$ "storage buffer" (20 mM Trizma Base, $150 \mathrm{mM} \mathrm{NH}_{4} \mathrm{Cl}$, pH set to 7.5 with $\mathrm{HCl}$ ) at $4{ }^{\circ} \mathrm{C}$ while stirring. After $6 \mathrm{~h}$ the buffer was refreshed and this was left overnight. The content of the dialysis bags was collected and concentrated to $1 \mathrm{~mL}$ using the $100 \mathrm{kDa}$ MWCO Amicon spin filters.

Final purification was performed using FPLC (Äktapurifier (GE Healthcare Lief Sciences)), using a Superose 6 10/11 GL column (GE Healthcare Life Sciences). The fractions containing the encapsulin was collected and again concentrated to $1 \mathrm{~mL}$ with the $100 \mathrm{kDa}$ MWCO Amicon spin filters. 
This sample was used to determine the concentration and formation of particles using the Nanodrop system and dynamic light scattering (DLS) (Microtrac Nanotract Wave W3043), respectively. For the DLS analysis the viscosity and refractive index of water were used and for the encapsulin a refractive index of 1.54 was used.

\subsubsection{Cryo-EM Analysis of BlE-DyP}

Purified BlE-DyP particles ( $5 \mu \mathrm{L}$ ) were applied to R2/2 300 mesh copperrhodium grids (Quantifoil Micro Tools, Germany) and vitrified using a FEI Vitrobot cryofixation unit. Data were collected on a FEI Titan Krios electron microscope operating at $300 \mathrm{kV}$ and images recorded on a Gatan Bio-quantum K2 summit detector at a calibrated magnification of 50,000 yielding a pixel size of $1.047 \AA$. Detector is integrated into FEI's automated data acquisition EPU software. A dose rate of 5.55 electrons $/ \AA^{2} / \mathrm{s}$ and $7 \mathrm{~s}$ exposure time were used to record 3837 70-frame movies with a defocus range of -1.0 to $-3.2 \mu \mathrm{m}$ in $0.2 \mu \mathrm{m}$ intervals.

To correct for beam-induced movement, frames of each movie were aligned using whole-image motion correction. ${ }^{18}$ Contrast transfer function parameters of each averaged movie were determined using CTFFIND4. ${ }^{19}$ Images showing astigmatism and/or motion signs were discarded, maintaining a total of 3,739. General image processing operations were performed using Xmipp ${ }^{20}$ and Relion. ${ }^{21}$ A total of 316,007 particles were picked with the Xmipp automatic picking routine. Alternatively, Relion referencefree $2 \mathrm{D}$ classification was used to discard bad particles and a similar data set of 291,878 particles was obtained. 3D classification with Relion resulted in four classes using a BlE-DyP low resolution map and low-pass filtered to $30 \AA$ as initial reference. Final Relion iterative auto-refine was performed including 271,489 particles (for the best 3D class). The best resolution, $2.65 \AA$ based on the gold-standard FSC $=0.143$ criterion corrected for the effects of a soft mask on the FSC curve using high- 
resolution noise substitution, was obtained using the complete data set of 3D-selected particles. ${ }^{22}$ The final density map was corrected for the modulation transfer function of the detector and sharpened by applying the estimated B-factor. ${ }^{23}$ Local resolution variations were calculated using MonoRes. ${ }^{24}$

The polypeptide chain in a single asymmetric unit of the $2.65 \AA$ cryoEM map was built using Coot crystallographic modelling ${ }^{25}$ and PHENIX real-space refinement tool. ${ }^{26}$ The comparison of predicted and observed SSE was used as starting point to register the amino acid sequence for encapsulin. Predicted SSE were determined by PSIPRED. Predicted quaternary structure was obtained by Swiss Model server. In this process, the T. maritima quaternary structure was used as a template. Once the backbone was registered, positions of the side chains were adjusted manually and fit of the atomic model to the density map was improved by iterative cycles of model rebuilding using Coot and PHENIX. To evaluate and improve model accuracy, we used Molprobity integrated in PHENIX ${ }^{27}$ to reduce clashes, rotamers outliers and inconsistencies in geometry. In addition, the geometry of the model was tested iteratively with PHENIX validation tools, which helped to detect and correct residues outside allowed regions in the Ramachandran plot. The Ramachandran plot showed no residues in disallowed regions.

Graphics and structural alignment between encapsulins and phages were produced by UCSF Chimera. ${ }^{28}$ The electrostatic potential for the BlEDyP capsid was calculated using DelPhi software ${ }^{29}$ and surface-colored with UCFS Chimera. 


\subsection{References}

1. Sutter, M.; Boehringer, D.; Gutmann, S.; Günther, S.; Prangishvili, D.; Loessner, M. J.; Stetter, K. O.; Weber-Ban, E.; Ban, N. Structural Basis of Enzyme Encapsulation into a Bacterial Nanocompartment. Nat, Struct. Mol. Biol. 2008, 15, 939-947

2. Giessen, T. W.; Orlando, B. J.; Verdegaal, A. A.; Chambers, M. G.; Gardener, J.; Bell, D. C.; Birrane, G.; Liao, M.; Silver, P. A. Structure and Function of a 9.6 Megadalton Bacterial Iron Storage Compartment. bioRxiv. 2019

3. Putri, R. M.; Allende-Ballestero, C.; Luque, D.; Klem, R.; Rousou, K. A.; Liu, A.; Traulsen, C. H.; Rurup, W. F.; Koay, M. S. T.; Castón, J. R.; Cornelissen, J. J. L. M. Structural Characterization of Native and Modified Encapsulins as Nanoplatforms for in Vitro Catalysis and Cellular Uptake. ACS Nano 2017, 11, 12796-12804

4. Williams, E. M.; Jung, S. M.; Coffman, J. L.; Lutz, S. Pore Engineering for Enhanced Mass Transport in Encapsulin Nanocompartments. ACS Synth. Biol. 2018, 7, 2514-2517

5. McHugh, C. A.; Fontana, J.; Nemecek, D.; Cheng, N.; Aksyuk, A. A.; Heymann, J. B.; Winkler, D. C.; Lam, A. S.; Wall, J. S.; Steven, A. C.; Hoiczyk, E. A Virus Capsid-like Nanocompartment that Stores Iron and Protects Bacteria from Oxidative Stress. EMBO J. 2014, 33, 18961911

6. Künzle, M.; Mangler, J.; Lach, M.; Beck, T. Peptide-directed Encapsulation of Inorganic Nanoparticles into Protein Containers. Nanoscale. 2018, 10, 22917-22926

7. Lau, Y. H.; Giessen, T. W.; Altenburg, W. J.; Silver, P. A. Prokaryotic Nanocompartments form Synthetic Organelles in a Eukaryote. Nat. Comm. 2018, 9, 1311 
8. Sigmund, F.; Massner, C.; Erdmann, P.; Stelzl, A.; Rolbienski, H.; Desai, M.; Bricault, S.; Wörner, T. P.; Snijder, J.; Geerlof, A.; Fuchs, H.; Hrabě de Angelis, M.; Heck, A. J. R.; Jasanoff, A.; Ntziachristos, V.; Plitzko, J.; Westmeyer, G. G. Bacterial Encapsulins as Orthogonal Compartments for Mammalian Cell Engineering. Nat. Commun. 2018, 9, 1990

9. Tamura, A.; Fukutani, Y.; Takami, T.; Fujii, M.; Nakaguchi, Y.; Murakami, Y.; Noguchi, K.; Yohda, M.; Odaka, M. Packaging Guest Proteins into the Encapsulin Nanocompartment from Rhodococcus erythropolis N771. Biotechnol. Bioeng. 2015, 112, 13-20

10. Rahmanpour, R.; Bugg, T. D. Assembly in Vitro of Rhodococcus jostii RHA 1 Encapsulin and Peroxidase DypB to Form a Nanocompartment. FEBS. 2013, 280, 2097-2104

11. Snijder, J.; Kononova, O.; Barbu, I. M.; Uetrecht, C.; Rurup, W. F.; Burnley, R. J.; Koay, M. S. T.; Cornelissen, J. J. L. M.; Roos, W. H.; Barsegov, V.; Wuite, G. J. L.; Heck, A. J. R. Assembly and Mechanical Properties of the Cargo-Free and Cargo-Loaded Bacterial Nanocompartment Encapsulin. Biomacromolecules. 2016, 17, 2522 - 2529

12. Snijder, J.; van de Waterbeemd, M.; Damoc, E.; Denisov, E.; Grinfeld, D.; Bennett, A.; Agbandje-McKenna, M.; Makarov, A.; Heck, A. J. R. Defining the Stoichiometry and Cargo Load of Viral and Bacterial Nanoparticles by Orbitrap Mass Spectrometry. J. of Am. Chem. Com. 2014, 136, 7295-7299

13. Gertsman, I.; Gan, L.; Guttman, M.; Lee, K.; Speir, J. A.; Duda, R. L.; Hendrix, R. W.; Komives, E. A.; Johnson, J. E. An Unexpected Twist in Viral Capsid Maturation. Nature. 2009, 458, 646 - 650

14. Gan, L.; Speir, J. A.; Conway, J. F.; Lander, G.; Cheng, N.; Firek, B. A.; Hendrix, R. W.; Duda, R. L.; Liljas, L.; Johnson, J. E. Capsid Conformational Sampling in HK97 Maturation Visualized by X-ray Crystallography and Cryo-EM. Structure. 2006, 14, 1655 - 1665 
15. Akita, F.; Chong, K. T.; Tanaka, H.; Yamashita, E.; Miyazaki, N.; Nakaishi, Y.; Suzuki, M.; Namba, K.; Ono, Y.; Tsukihara, T.; Nakagawa, A. The Crystal Structure of a Virus-like Particle from the Hyperthermophilic Archaeon Pyrococcus Furiosus Provides Insight into the Evolution of Viruses. J. Mol. Biol. 2007, 368, 1469 - 1483

16. Hryc, C. F.; Chen, D. H.; Afonine, P. V.; Jakana, J.; Wang, Z.; Haase-Pettingell, C.; Jiang, W.; Adams, P. D.; King, J. A.; Schmid, M. F.; Chiu, W. Accurate Model Annotation of a Near-atomic Resolution Cryo-EM Map. Proc. Natl. Acad. Sci. USA. 2017, 114, 3103 - 3108

17. Rurup, W. F.; Snijder, J.; Koay, M .S. T.; Heck, A. J. R.; Cornelissen, J. J. L. M. Self-Sorting of Foreign Proteins in a Bacterial Nanocompartment. JACS. 2014, 136, 3828 - 3832

18. Li, X.; Mooney, P.; Zheng, S.; Booth, C. R.; Braunfeld, M. B.; Gubbens, S.; Agard, D. A.; Cheng, Y. Electron Counting and Beaminduced Motion Correction Enable Near-atomic-resolution Single-particle Cryo-EM. Nature Methods. 2013, 10, 584-590

19. Rohou, A.; Grigorieff, N. CTFFIND4: Fast and Accurate Defocus Estimation from Electron Micrographs. J. Struct. Biol. 2015, 192, 216-221

20. de la Rosa-Trevín, J. M.; Otón, J.; Marabini, R.; Zaldívar, A.; Vargas, J.; Carazo, J. M.; Sorzano, C. O. S. Xmipp 3.0: An Improved Software Suite for Image Processing in Electron Microscopy. J. of Struct. Biol. 2013, 184, 321-328

21. Scheres, S. H. W. RELION: Implementation of a Bayesian Approach to Cryo-EM Structure Determination. J. Struct. Biol. 2012, $180,519-530$

22. Chen, S.; McMullan, G.; Faruqi, A. R.; Murshudov, G. N.; Short, J. M.; Scheres, S. H. W.; Henderson, R. High-resolution Noise Substitution to Measure Overfitting and Validate Resolution in 3D Structure Determination by Single Particle Electron Cryomicroscopy. Ultramicroscopy 2013, 135, 24-35 
23. Rosenthal, P. B.; Henderson, R. Optimal Determination of Particle Orientation, Absolute Hand, and Contrast Loss in Single-particle Electron Cryomicroscopy. J. Mol. Biol. 2003, 333, 721-745

24. Vilas, J. L.; Gómez-Blanco, J.; Conesa, P.; Melero, R.; de la RosaTrevín, J. M.; Otón, J.; Cuenca, J.; Marabini, R.; Carazo, J. M.; Vargas, J.; Sorzano, C. O. S. MonoRes: Automatic and Accurate Estimation of Local Resolution for Electron Microscopy Maps. Structure. 2018, 26, 337-344

25. Emsley, P.; Cowtan, K. Coot: Model-building Tools for Molecular Graphics. Acta Crystallogr. D. Biol. Crystallogr. 2004, 60, 2126-2132 26. Afonine, P. V.; Poon, B. K.; Read, R. J.; Sobolev, O. V.; Terwilliger, T. C.; Urzhumtsev, A.; Adams, P. D. Real-space Refinement in PHENIX for Cryo-EM and Crystallography. Acta Crystallogr. D. Biol. Crystallogr. 2018, 74, 531-544

27. Williams, C. J.; Headd, J. J.; Moriarty, N. W.; Prisant, M. G.; Videau, L. L.; Deis, L. N.; Verma, V.; Keedy, D. A.; Hintze, B. J.; Chen, V. B.; Jain, S.; Lewis, S. M.; Arendall, W. B. $3^{\text {rd }}$; Snoeyink, J.; Adams, P. D.; Lovell, S. C.; Richardson, J. S.; Richardson, D. C. MolProbity: More and Better Reference Data for Improved All-atom Structure Validation. Protein Sci. 2018, 27, 293-315

28. Pettersen, E. F.; Goddard, T. D.; Huang, C. C.; Couch, G. S.; Greenblatt, D. M.; Meng, E. C.; Ferrin, T. E. UCSF Chimera-a Visualization System for Exploratory Research and Analysis. J. Comput. Chem. 2004, 25, 1605-1612

29. Rocchia, W.; Sridharan, S.; Nicholls, A.; Alexov, E.; Chiabrera, A.; Honig, B. Rapid Grid-based Construction of the Molecular Surface and the Use of Induced Surface Charge to Calculate Reaction Field Energies: Applications to the Molecular Systems and Geometric Objects. J. Comput. Chem. 2002, 23, 128-137 
Chapter 3

$m$ 


\section{Chapter 4}

\section{Construction and Evaluation}

of L-Asparaginase loaded

\section{Protein Cages}

\section{Abstract}

To explore the potential of protein nanocages in enzyme therapy we encapsulated L-asparaginase in two different protein cages. L-asparaginase depletes the blood of L-asparagine in the treatment of acute lymphoblastic leukemia. In this model system we used both the bacterial encapsulins and the cowpea chlorotic mottle virus (CCMV), which are isolated from virus-infected plants. As determined by cryo-EM, encapsulin- and CCMV-based cages have a porous structure of the protein shell. We show that the encapsulated enzymes have variable activity based on the nature of the protein cage and/or the enzyme cargo. The particles containing L-asparaginase induce in vitro cell death in acute lymphoblastic leukemia cells. The results show the potential of using enzymes encapsulated in protein nanocages, combining the enzymatic activity with the versality of the protein nanocages.

R. Klem, M. V. de Ruiter, V. M. Dombrowe, A. Juan, D. Gil-Cantero, C. AllendeBallestero, D. Luque, J. R. Castón, J. J. L. M. Cornelissen; "Activity and Leukemia Cell Viability of Asparaginase-loaded Protein Cages". Manuscript submitted. 


\subsection{Introduction}

In Nature a variety of protein nanocages is found with different functions; for example, viruses employ protein cages to deliver genetic material to host cells and bacterial nanocompartments sequester part of a metabolic pathway. In artificial systems these well-defined protein architectures have been used in, amongst others, materials science and biomedical applications. In the latter application the distinct size and shape, ability to modify the surface and cargo and biocompatibility potentially gives advantages in delivery, targeting and stability of encased therapeutic enzymes.

The enzyme L-asparaginase (ASNase) is prescribed to treat acute lymphoblastic leukemia (ALL), a type of cancer that is found predominantly in children. ${ }^{1,2}$ ASNase catalyses the reaction from L-asparagine to Laspartate and, to a lesser degree, L-glutamine to L-glutamate. ${ }^{3}$ Contrary to most other cells in the human body, ALL cells have reduced levels of asparagine synthetase and rely on extracellular uptake for L-asparagine supply. ${ }^{4,5}$ Therefore, intravenous injection of ASNase, which depletes Lasparagine in the blood, can treat ALL. The cells are depleted of Lasparagine; they can neither produce it nor take it up. This, along with the reduced L-glutamine levels, results in specific cell death; this therapy selectively kills ALL cells, but non-ALL cells are unharmed as they can convert L-aspartate to L-asparagine through asparagine synthetase. ${ }^{6}$ ASNase formulations have been improved by using various bacterial strains as origins ${ }^{1,2}$ and by PEGylating the enzyme. ${ }^{7}$ However, low bioavailability, immunogenicity and toxicity ${ }^{8,9}$ remain challenges to an optimal therapy.

One option to solve some of these drawbacks, is to encapsulate ASNase in a protein nanocage. The nanocage can be modified on the exterior to create functional nanocages for specific targeting, ${ }^{10,11,12}$ higher substrate specificity ${ }^{13,14}$ and reduced immunogenicity. ${ }^{15,16,17} \mathrm{~A}$ variety of protein 
nanocages are used for encapsulating a broad range of enzymes, ${ }^{18}$ including virus-like particles, ${ }^{19,20}$ lumazine synthase capsids ${ }^{13,21}$ and ferritin. ${ }^{22}$ These protein nanocages offer high stability and reduce the toxicity of their cargo, ${ }^{19,21,23}$ while the nanocages are often porous, which allows transport of substrates and products across the protein shell.

To explore the potential of protein nanocages in enzyme therapy we encapsulated the enzyme ASNase into protein cages. We used both the cowpea chlorotic mottle virus (CCMV) capsid, which is isolated from CCMVinfected plants, and the bacterial encapsulin. The resulting nanocages are monodisperse with diameters of 20 and $24 \mathrm{~nm}$, respectively.

CCMV, with a $\mathrm{T}=3$ icosahedral structure,${ }^{24}$ can be used for enzyme delivery. CCMV is easily and reversibly disassembled into its subunits and reassembled into an empty or filled virus-like particle (VLP) by changing the $\mathrm{pH}$ and salt concentration. ${ }^{25,26,27}$ This self-assembly mechanism has been exploited to encapsulate enzymes such as glucose oxidase, ${ }^{28}$ peroxidase $^{29}$ and cytochrome P450. ${ }^{30}$ CCMV has a broad biodistribution and low toxicity in vivo and has a minimal chance of replication in mammalian cells, because of its plant origin.

Encapsulins are found in various bacteria, are $\sim 24 \mathrm{~nm}$ in size and consist of 60 monomers forming highly stable $\mathrm{T}=1$ or $\mathrm{T}=3$ particles. ${ }^{31}$ They naturally contain protein cargo, such as dye decolorizing peroxidase or ferritin-like protein, but they vary in cargo and function. ${ }^{32}$ In bacteria, encapsulins often function as nanocompartments that act like organelles. The sturdy nature of encapsulins potentially assists in stabilizing the cargo enzymes and maintaining a specific environment; they remain stable at both high and low $\mathrm{pH}$. The cargo can be modified by changing the genetic sequence of the operon that encodes for the cargo and encapsulin monomers, provided that the C-terminus sequence of the cargo, which 
links the cargo to the shell, remains unaltered. ${ }^{33,34}$ We use encapsulins derived from Brevibacterium linens (BlE), although many other bacteria contain similar protein nanocages. ${ }^{32}$

In this research we introduce the medicinally relevant ASNase encapsulated in two protein nanocages of both viral (CCMV) and bacterial origin (BlE). The activity of encapsulated ASNase in encapsulin and CCMVbased cages is compared with native ASNase. Furthermore, we evaluate their ability to induce cell death in ALL cells to show their potential for the treatment of ALL. We show that the encapsulated enzyme remains active and that the particles can induce cell death in the ALL cells using low $\mathrm{nM}$ or even $\mathrm{pM}$ concentrations. The results show the potential of using enzymes encapsulated in protein nanocages for medical use.

\subsection{Results and Discussion}

\subsubsection{Production, Purification and Analysis of En- capsulated ASNase}

We designed the ASNase that was encapsulated in Brevibacterium linens encapsulin (BlE-ASNase) by genetic substitution of the native BlE cargo (dye decolorizing peroxidase (DyP)) for the genetic sequence encoding for the ASNase Type I monomer (Chapter 3). The filled encapsulins were expressed in and purified from Escherichia coli (E. coli) bacteria using the protocol designed by Rurup et al. ${ }^{35,36}$ They were further purified using size exclusion chromatography (SEC) (Figure 4.1A). The fractions corresponding to the peak at an elution volume of $12.5 \mathrm{~mL}$ were isolated and analysed by dynamic light scattering (DLS), which showed the particles were sized $\sim 20 \mathrm{~nm}$, which corresponds to $\mathrm{T}=1$ particles (Figure 4.1B). The BlE-ASNase assembly was further confirmed by transmission electron microscopy (TEM) of negatively stained particles (Figure 4.1C). 


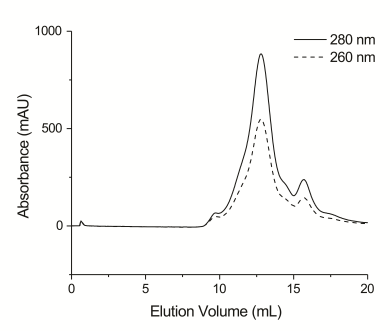

(a)

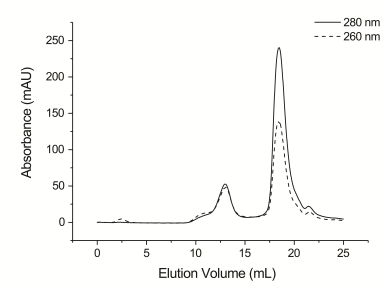

(d)

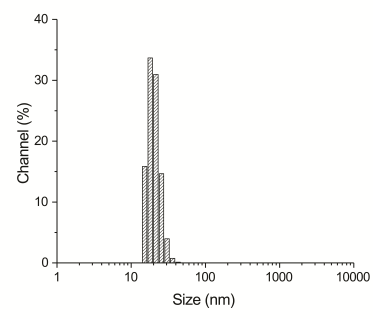

(b)

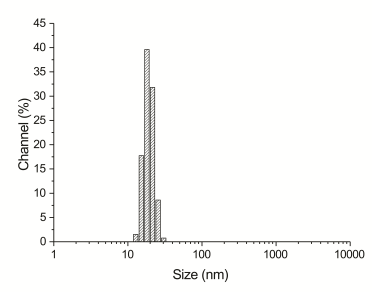

(e)

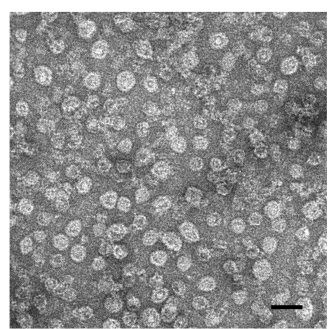

(c)

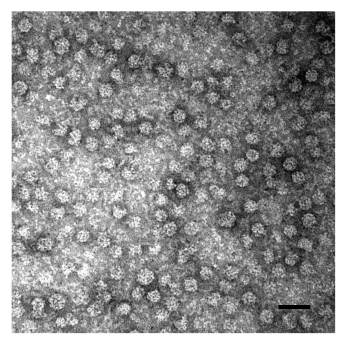

(f)

Figure 4.1: Characterization of the ASNase-filled particles, for BlEASNase: SEC (A), DLS (B) and TEM (C). For CCMV-ASNase: SEC (D), DLS (E) and TEM (F). Scale bars in C and F are $50 \mathrm{~nm}$.

To make the CCMV-ASNase complex, we used commercial ASNase from E. coli and native CCMV, which we produced and isolated from the cowpea plant. We removed its native RNA cargo using a protocol described in literature. ${ }^{29}$ To effectively encapsulate new cargo in CCMV, a sufficient negative charge is required to induce the cargo encapsulation. Therefore, the ASNase was modified with $20 \mathrm{kDa}$ polystyrene sulfonate (PSS) polymers using an NHS-Maleimide heterobifunctional linker. This PSS-modified ASNase (ASNase-PSS) was purified using SEC, and subsequently mixed with free CCMV capsid protein dimers to assemble spontaneously. This was again purified using SEC (Figure 4.1D), in which the $12 \mathrm{~mL}$ elution volume peak corresponds to the virus-sized structures 
and the $18 \mathrm{~mL}$ elution volume peak corresponds to the free capsid proteins. For CCMV, uniform monodisperse particles of $\sim 19 \mathrm{~nm}$ were found using DLS (Figure 4.1E) and TEM (Figure 4.1F), which corresponds to a size that was reported for an icosahedral CCMV particle with $\mathrm{T}=1$ symmetry. ${ }^{28}$

The purified BlE-ASNase and CCMV-ASNase were further analysed using sodium dodecyl sulfate polyacrylamide gel electrophoresis (SDS-PAGE) to determine the composition of the proteins (Figure $4.2 \mathrm{~A}$ and $\mathrm{B}$ ) and verify that the nanocages both contained ASNase. 


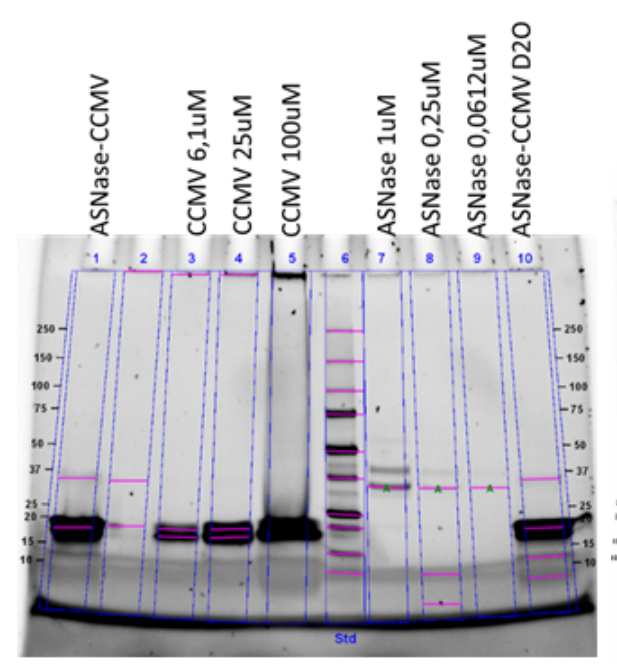

(a)

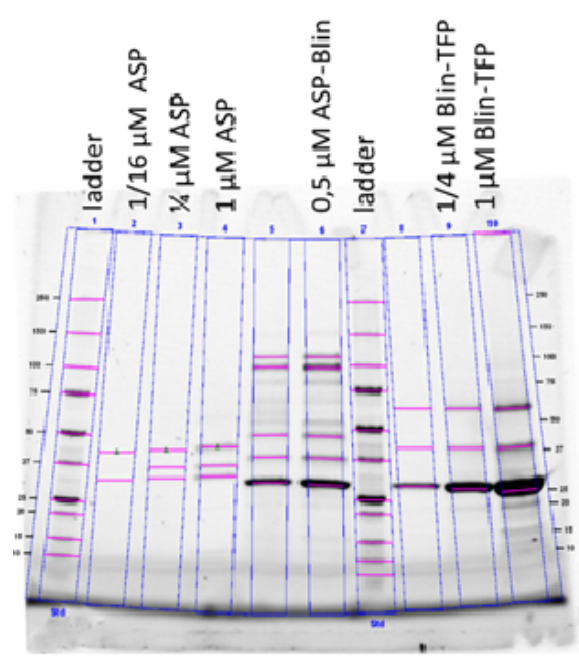

(b)

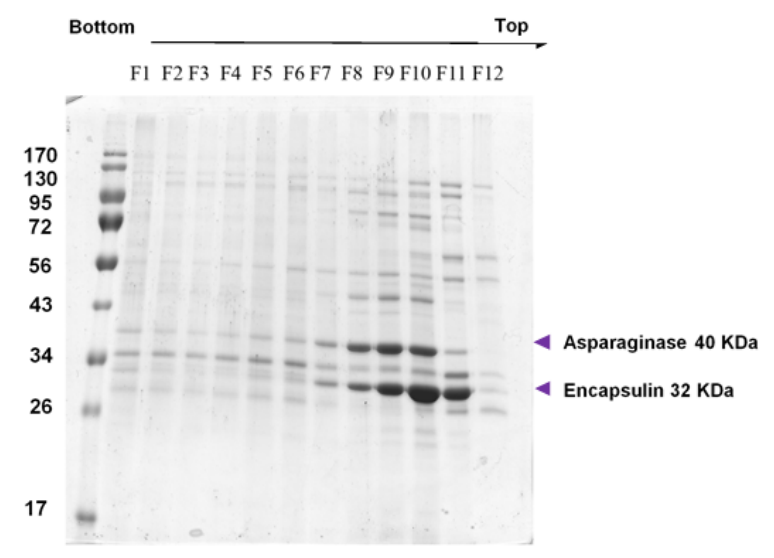

(c)

Figure 4.2: SDS-PAGE on a 4 to $15 \%$ stain-free gel for (A) CCMVASNase and (B) BlE-ASNase. We used Precision Plus Protein Standards (Bio-Rad) as ladder. The gels were analysed with Image Lab software. (C) SDS-PAGE of BlE-ASNase after repurification by a $20-50 \%$ sucrose gradient. We used fraction 10 for the cryo-EM analysis. 


\subsubsection{ELISA of Encapsulated ASNase}

We performed an enzyme-linked immunosorbent assay (ELISA) to assure that ASNase was not present in the solution after purifying the BlE and CCMV or that it was bound to the exterior of the nanocages (Figure 4.3). Non-encapsulated ASNase showed a signal that is concentration dependent. The blank (air) and the negative control showed no signal, which indicates that the primary anti-ASNase antibody binds specifically. The BlE-ASNase and CCMV-ASNase samples also showed no increased absorbance. Thus, the samples are stable and the ASNase is protected within the cage and cannot be reached by the antibody. The ASNase-PSS showed similar absorbance compared to the native enzyme. The PSS does not block the primary antibody from binding ASNase. From the absence of a signal for both the BlE-ASNase and CCMV-ASNase, we conclude that there is no detectable free enzyme present in the solution and thus that any observed activity must be the result of encapsulated ASNase.

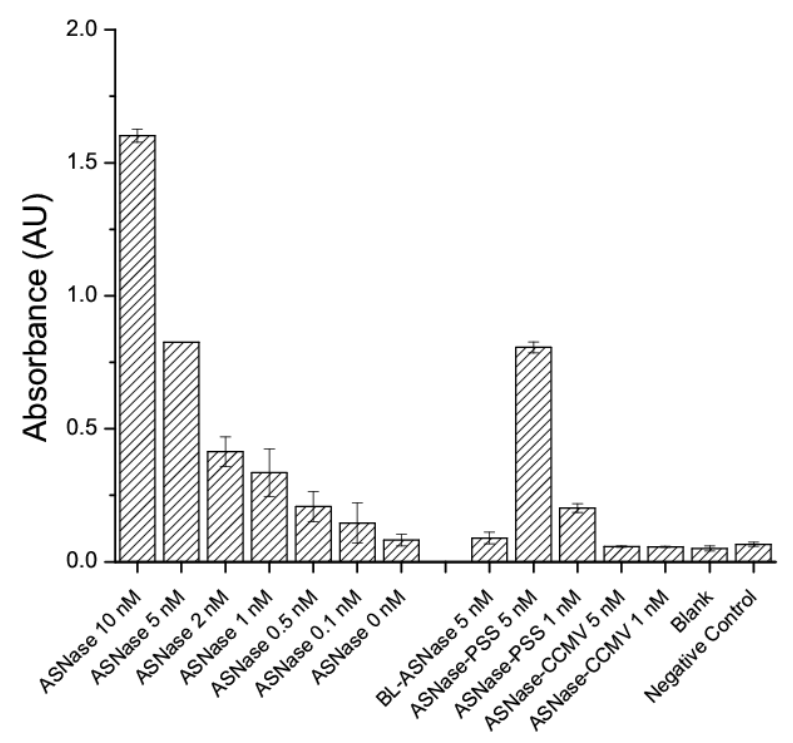

Figure 4.3: ELISA of native ASNase, BlE-ASNase and ASNase-PSS, both native and encapsulated in CCMV. 


\subsubsection{Cryo-EM Reconstruction of ASNase loaded Par- ticles}

We used cryo-EM to analyse the structures of both BlE-ASNase and CCMV-ASNase particles and we obtained a 3D reconstruction (3DR) for each of the two sets of particles (Figures 4.4 and 4.5). In addition to the loaded encapsulin particles (Figure 4.4A, white arrows), BlE-ASNase samples contained heterogeneous vesicle-like structures (Figure 4.4A, white arrowheads) that we discarded. Based on a 0.143 Fourier shell correlation (FSC) threshold, the resolution was $4.5 \AA$ for BlE-ASNase. The outer diameter, determined from spherically averaged radial density plots of the 3DR, was $236 \AA$ and the inner diameter was $190 \AA$.

The encapsulin map is an icosahedral shell with an average thickness of $23 \AA$ and the particle is based on a $\mathrm{T}=1$ lattice. The capsid-like particle is formed by 12 slightly outward-protruding pentamers, with numerous rod-like structures that correspond to $\alpha$-helices (Figure 4.4B). The particles have numerous relatively small channels, $\sim 5-8 \AA$ in diameter, that extend through the protein shell, located at the two-, three-, and fivefold axes, as well as around them (Figure 4.4C). Docking analysis showed that BlE map was similar to the $\mathrm{T}=1$ encapsulin map of Thermotoga maritima (TmE). The docked TmE crystallographic model (PDB entry 3DKT) matched with the secondary-structure elements of the cryo-EM map (Figure 4.4D), indicating a similar fold for both encapsulins. In addition, most pores in the TmE shell colocalized with the pores in BlE. To infer ASNase-related cargo organization, 3DR of BlE-ASNase particles were calculated without imposing icosahedral symmetry. The resulting map (average resolution $\sim 12 \AA$ ) showed an irregular cargo density with major connections to the inner surface of BlE close to the three-fold axis (Figure 4.4E, arrow). While at least one tetrameric ASNase (PDB 3ECA) is docked in this internal density, the internal volume of $\mathrm{BlE}\left(\sim 3.6 \cdot 10^{6}\right.$ $\AA^{3}$ ) suggests that additional copies of ASNase can be encapsulated. 


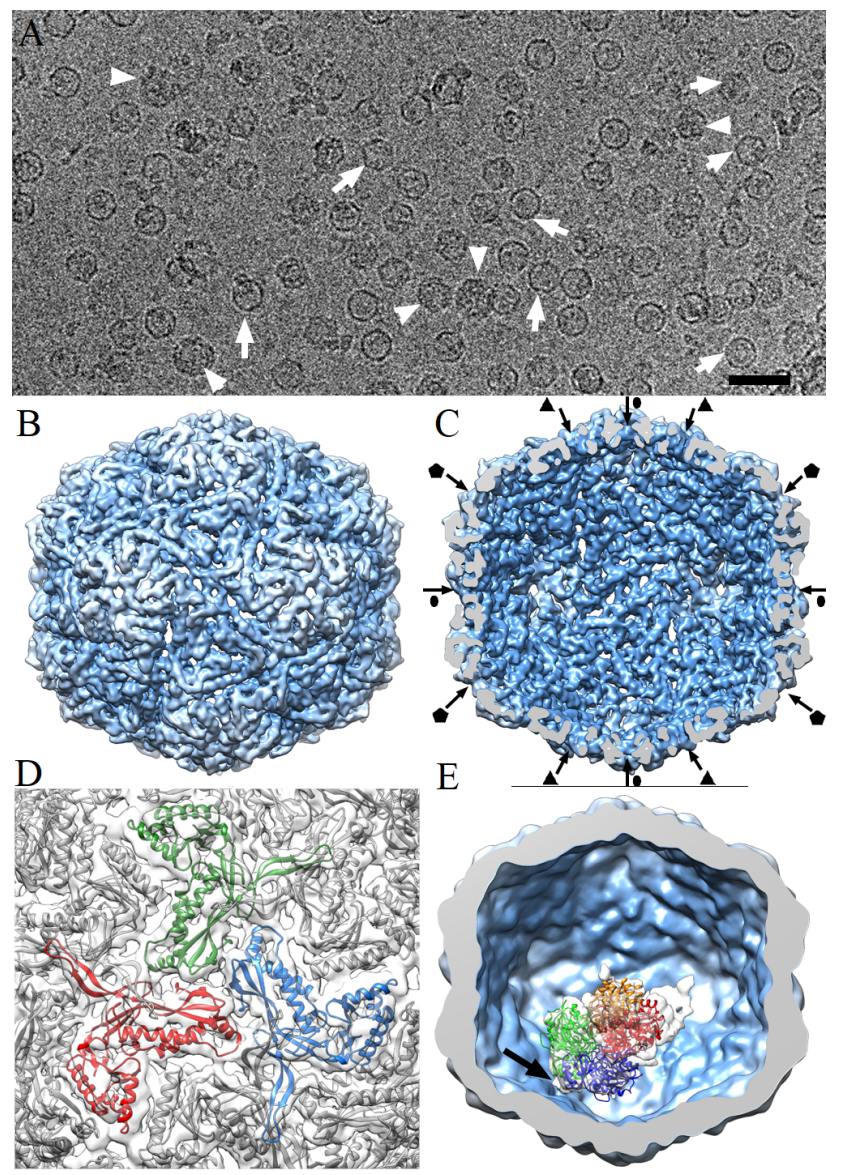

Figure 4.4: (A) Cryo-EM image of ASNase-loaded encapsulin. Arrows indicate BlE-ASNase; arrowheads point to vesicle-like material. Bar = $500 \AA$ A. (B, C) Radial color-coded map with icosahedral symmetry of BlEASNase viewed along a two-fold symmetry axis contoured at $4 \sigma$ above mean density. Outer (B) and inner (C) surfaces are shown. Arrows indicate pores at the five-, three- and two-fold axes ( $\bullet, \triangle$ and $\circ$, respectively). (D) BlE-ASNase outer surface viewed along a three-fold axis, with docked TmE atomic coordinates. Encapsulin monomers at the three-fold axis are depicted in red, green and blue. (E) Radial color-coded map without symmetry of BlE-ASNase from inside, with docked ASNase tetramer, contoured at $2.5 \sigma$ above the mean density. Arrow indicates the major connection to the inner encapsulin surface at the three-fold axis. 
CCMV-ASNase samples analysed by cryo-EM contained homogeneous spherical particles (Figure 4.5A). Two-and three-dimensional classification methods resulted in an icosahedral capsid with a $\mathrm{T}=1$ lattice of $200 \AA$ in diameter, formed by 60 capsid protein subunits. Although the number of particles included in the 3D map was very large, the resolution for the CCMV-ASNase capsid was limited to $5.6 \AA$ (0.143 FSC threshold), suggesting a high structural flexibility for the icosahedral shell. The capsid has large pores at the three-fold axes with a triangular shape (50 $\AA$ long each side), and at the five-fold axes the pores are $20 \AA$ in diameter (Figure 4.5B). After imposing icosahedral symmetry, the cryo-EM map had a spherical density in the capsid interior with a $\sim 60 \AA$ radius that corresponds to the packed tetrameric ASNase (Figure 4.5C). Secondary structural elements were identified by docking the CCMV capsid protein $\mathrm{X}$-ray map (PDB entry 1CWP) into the cryo-EM map. The concordance of the two maps is clear except for the C-terminal end (residues 179-190) of the capsid protein, which was adjusted to a neighbouring cryo-EM density. CCMV capsid protein dimers are the building blocks of this cage (containing 30 dimers), with a well-defined dimeric interaction surface. The hinge angle formed between capsid protein dimers was $48^{\circ}$ (Figure 4.5E). In the swollen $\mathrm{T}=3 \mathrm{CCMV}$ capsid and in other phthalocyanineand glucose oxidase-loaded $\mathrm{T}=1$ capsids, the hinge angle is $\sim 60^{\circ}$, and the dimeric contacts are diminished. ${ }^{28,37}$ This suggests that the cargo affects the dimer organization of CCMV, which could influence the whole CCMV structure. 

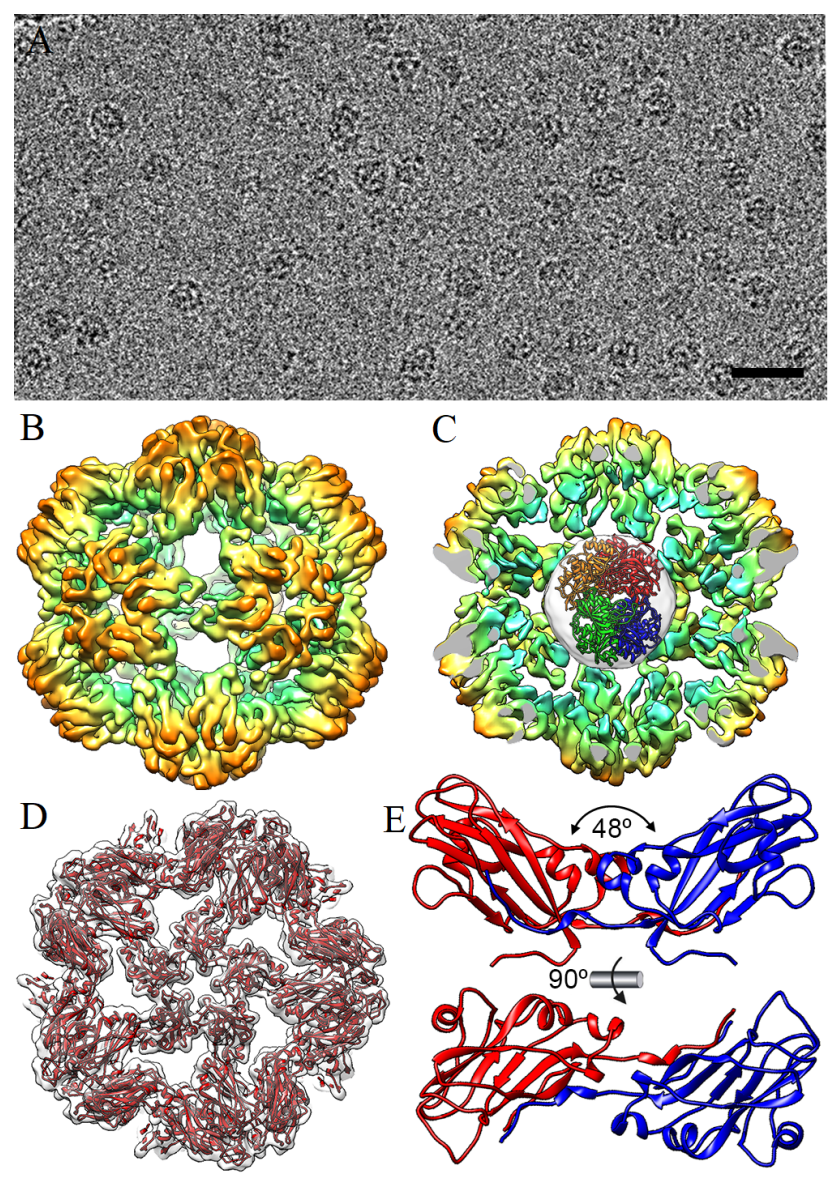

Figure 4.5: (A) Cryo-EM image of CCMV-ASNase particles. Bar = $500 \AA$. (B, C) Radial color-coded map with icosahedral symmetry of CCMV-ASNase viewed along a two-fold axis of symmetry contoured at 4 $\sigma$ above the mean density. Outer (B) and inner (C) surfaces are shown. CCMV-ASNase from inside shows docked ASNase tetramer in the internal density. (D) CCMV-ASNase viewed down a five-fold axis from outside, with docked CCMV protein atomic coordinates (red). (E) Capsid protein dimers in CCMV-ASNase $\mathrm{T}=1$ capsids. Side view (top), top view (bottom). The hinge dihedral angle is indicated. 


\subsubsection{Activity of Encapsulated ASNase}

We used proton nuclear magnetic resonance spectroscopy $\left({ }^{1} \mathrm{H}\right.$ NMR $)$ to monitor the catalytic reaction, quantify the produced L-aspartate and extract kinetic information. L-asparagine does not convert to L-aspartate in $\mathrm{D}_{2} \mathrm{O}$ in the absence of ASNase (Figure 4.6). The ${ }^{1} \mathrm{H}$ NMR spectra of L-asparagine and L-aspartate show different chemical shifts, therefore, the catalytic reaction can be monitored over time by ${ }^{1} \mathrm{H}$ NMR spectroscopy. The activity of the enzyme-constructs was monitored upon the addition of $10 \mathrm{mM}$ L-asparagine to various concentrations ( $1 \mathrm{nM}$ to $1 \mu \mathrm{M}$ ) of ASNase. The average turnover number $\left(\mathrm{K}_{\text {cat }}\right)$ of the free ASNase was $42 \pm 6 \mathrm{~s}^{-1}$ under the set conditions (Figure $4.7 \mathrm{~A}$ ).

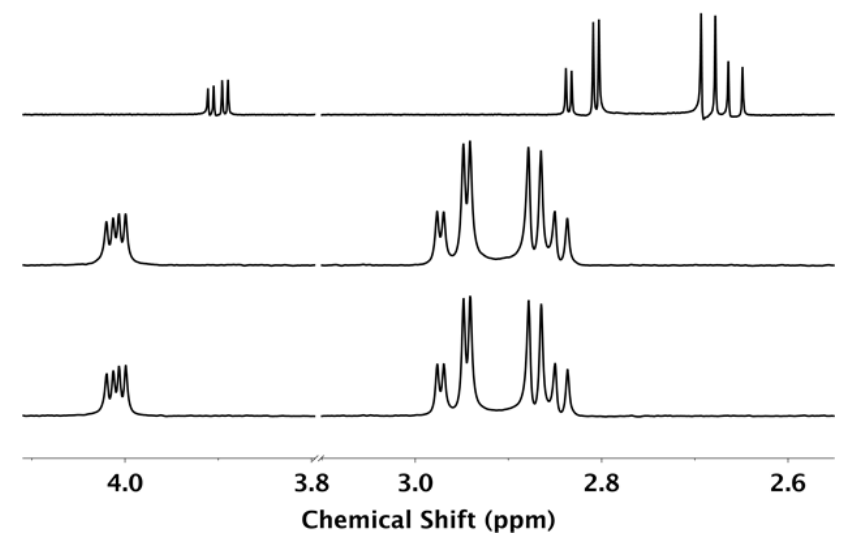

Figure 4.6: Selected region of ${ }^{1} \mathrm{H}$ NMR overlaid spectra of: $10 \mathrm{mM} \mathrm{L}-$ asparagine (bottom); $10 \mathrm{mM}$ L-asparagine after $24 \mathrm{~h}$ (middle); $10 \mathrm{mM}$ L-aspartate (top). All analyses were performed in $\mathrm{D}_{2} \mathrm{O}$. With ${ }^{1} \mathrm{H} \mathrm{NMR}$, we found a clear difference in the chemical shift between the substrate (Lasparagine, bottom) and the product (L-aspartate, top) of the conversion catalysed by ASNase. Moreover, $24 \mathrm{~h}$ incubation of L-asparagine (middle) without addition of ASNase does not affect the NMR spectrum; there is no conversion of L-asparagine to L-aspartate in the absence of ASNase. 


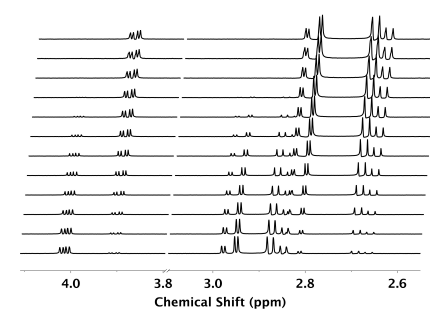

(a)

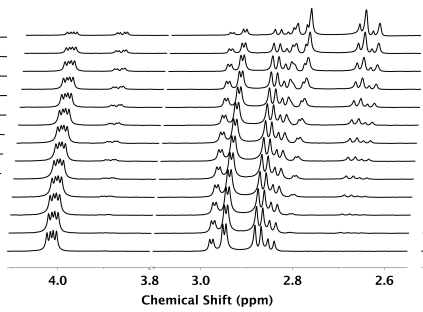

(b)

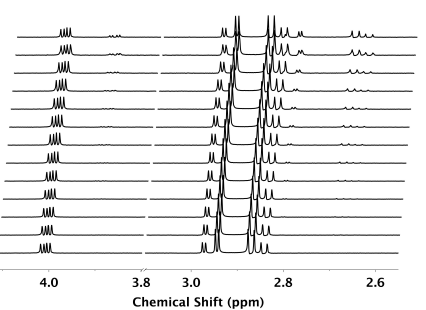

(c)

Figure 4.7: ${ }^{1} \mathrm{H}$ NMR spectra showing the conversion of L-asparagine to L-aspartate as catalyzed by (A) native ASNase, (B) CCMV-ASNase and (C) BlE-ASNase. The bottom line is at $t=0$ with time increasing to $t$ $=22 \mathrm{~h}$ at the top.

Once the ASNase is modified by the PSS polymer, the $\mathrm{K}_{\text {cat }}$ decreases to $3.5 \pm 0.1 \mathrm{~s}^{-1}$. Stephanopoulos et al. showed that modification of enzymes can reduce its activity. ${ }^{38}$ This is often caused by changes to the tertiary structure of the enzyme or the partial blocking of the active site. When ASNase is encapsulated in CCMV, the $\mathrm{K}_{\text {cat }}$ was $5.2 \pm 0.3 \mathrm{~s}^{-1}$ (Figure 4.7B). This is lower than the native ASNase but showed increased activity compared with non-encapsulated ASNase-PSS. These differences in activity might be caused by stabilization of the enzyme by the capsid, as seen previously for other VLP systems, but can also be caused by a different local environment ${ }^{19}$ (e.g., $\mathrm{pH}$, salt, and/or specific cations). However, BlE-ASNase had a highly decreased conversion speed of $\mathrm{K}_{\text {cat }}=0.0017 \mathrm{~s}^{-1}$ (Figure $4.7 \mathrm{C}$ ).

The lower conversion with BlE-ASNase indicates that the activity is reduced after converting $5 \mathrm{mM}$ L-asparagine (Table 4.1). After $2 \mathrm{~h}, 5 \mathrm{mM} \mathrm{L}-$ asparagine is converted regardless of the substrate starting concentration, which is slower compared to non-encapsulated ASNase. In the following $22 \mathrm{~h}$, a significantly smaller amount of L-asparagine is converted (Table 4.1). The protein shell forming the encapsulin limits the passage of L- 
asparagine to the encapsulated enzyme, as opposed to the freely available ASNase that has no protein shell surrounding it. The charge distribution on the walls of the narrow channels can be a major limiting factor. However, the significantly reduced activity after $2 \mathrm{~h}$ in the presence of BlE-ASNase suggests other reasons for this reduced activity, which is potentially caused by product inhibition from L-aspartate remaining within the encapsulin (Table 4.1). The negative charge surrounding the small pores of the encapsulin (Chapter 3) can limit the passage of L-aspartate, resulting in an increased local concentration. This diffusion limitation was not observed for the CCMV-ASNase complex, presumably because of the substantially larger pores in the CCMV shell (Figure $4.5 \mathrm{~B}-\mathrm{C}$ ). ${ }^{28,39}$

Table 4.1: Conversion of different concentrations of L-asparagine to Laspartate by BlE-ASNase after 2 and 24 hours.

\begin{tabular}{c|cc|cc} 
& \multicolumn{2}{|c}{2 hours } & \multicolumn{2}{c}{24 hours } \\
\hline$[\text { Asn }]_{\text {initial }}(\mathrm{mM})$ & Asn (\%) & Asp (\%) & Asn (\%) & Asp (\%) \\
5 & 40 & 60 & 42.86 & 57.14 \\
10 & 93.33 & 6.66 & 54.72 & 45.28 \\
20 & 97.11 & 2.88 & 84.31 & 15.68
\end{tabular}

The percentage of converted L-asparagine depends its starting concentration (Table 4.1). When starting at $5 \mathrm{mM}, 60 \%$ of the substrate is converted after $2 \mathrm{~h}$, while for $10 \mathrm{mM}$ starting concentration, only $6.66 \%$ is converted and for $20 \mathrm{mM}$ it is $2.88 \%$. The exact percentages are not completely accurate, as the low concentrations determined by the integrals were on the lower limit of NMR detection. However, there is a clear difference between the starting concentrations that is large enough to infer that the first $\sim 1 \mathrm{mM}$ of L-asparagine is converted within $2 \mathrm{~h}$. Interestingly, with the $5 \mathrm{mM}$ starting concentration, the maximum conversion is 
reached within $2 \mathrm{~h}$, as the L-asparagine:L-aspartate ratio is the same after $24 \mathrm{~h}$. These data suggest that the conversion speed of L-asparagine to L-aspartate is slowing down over time and reaches (near) 0 at this ratio of 40:60 in the BlE-ASNase samples. This could be caused by product inhibition of the enzyme; the L-aspartate does not leave the BlE.

The differences in activity between BlE-ASNase and CCMV-ASNase can also be a result of the difference their synthesis; for CCMV-ASNase, ASNase is bought, mixed with capsid proteins and encapsulated, while for BlE-ASNase, the ASNase monomers and the encapsulin monomers are formed simultaneously. In E. coli, the encapsulin is formed together with the ASNase monomers; active tetramers of ASNase might not always have been formed when they are encapsulated in BlE. This can explain the reduced activity compared to CCMV-ASNase which encapsulates preformed, active ASNase tetramers. Another potential disadvantage is the 56 amino acid C-terminal segment that is covalently bound to ASNase, directing its encapsulation in BlE. This fragment might affect the enzyme structure which in turn could reduce its activity.

\subsubsection{Induction of ALL Cell Death by Encapsulated ASNase}

We studied the efficiency of encapsulated ASNase in inducing cell death in ALL cells over $72 \mathrm{~h}$ and compared it with non-encapsulated ASNase (Figure 4.8). We found that ASNase is effective in killing acute lymphoblastic leukemia (ALL) cells in the $\mathrm{pM}$ concentration range. Increasing the concentration over $25 \mathrm{pM}$ did not further decrease cell viability (P $<0.001)$ and not all cells died (24 h samples, Figure 4.8A). Depletion of L-asparagine does not instantly kill all ALL cells, but probably triggers a cell death mechanism that takes time. Thus, sustained L-asparagine reduction is preferred when used therapeutically. 


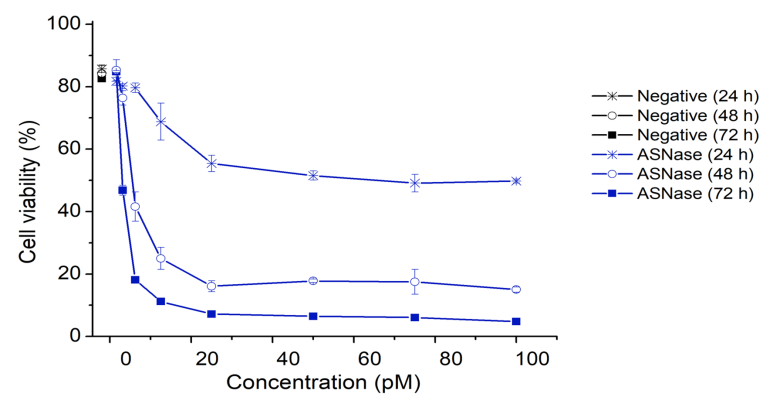

(a)

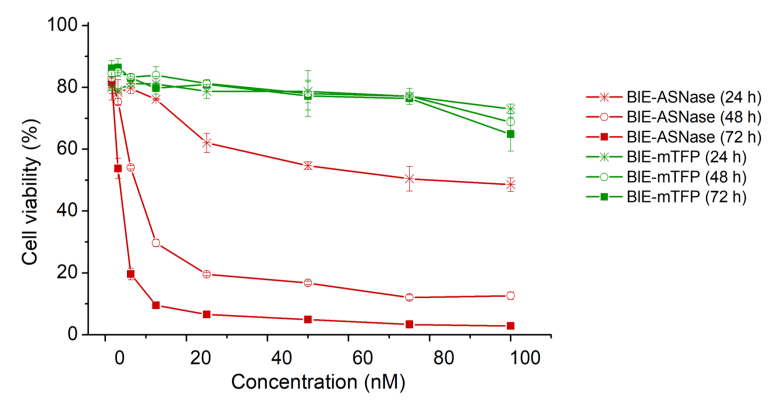

(b)

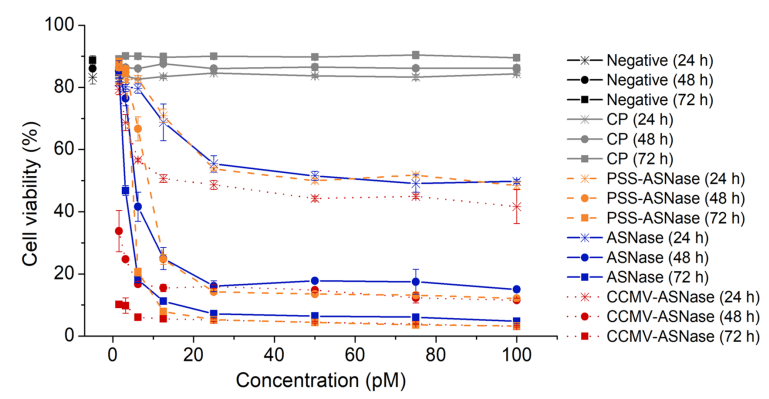

(c)

Figure 4.8: Cell viability after treatment with non-encapsulated ASNase (A), BlE-ASNase (B) and CCMV-ASNase (C). For BlE-ASNase (B), the $\mathrm{x}$-axis is in $\mathrm{nM}$ instead of $\mathrm{pM}$. We used BlE-mTFP as a control for BlE. BlE-mTFP is BlE with monomeric Teal Fluorescent Protein cargo. CP are the CCMV capsid proteins without ASNase cargo. ASNase-PSS is ASNase modified with PSS without the CCMV protein cage. 
When encapsulated in $\mathrm{BlE}$, ASNase activity is reduced and a 1,000x higher concentration (nM range as opposed to the $\mathrm{pM}$ range for native and CCMV-ASNase) is required for an effect similar to that produced by native ASNase (Figure 4.8A and B). However, increasing the concentration to more than $25 \mathrm{nM}$ does not increase cell death, which is probably caused by the product inhibition. Alternatively, it also suggests that ALL cell death probably is a process that takes time and adding more than a specific amount of ASNase is not beneficial to the therapy.

In contrast to BlE-ASNase, CCMV-ASNase induces cell death in ALL cells as effectively when compared with native ASNase (Figure $4.8 \mathrm{C}$ ). At the lowest concentration (up to $12.5 \mathrm{pM}$ ), CCMV-ASNase shows a higher induction of cell death compared with that of native ASNase (Figure 4.8A) $(\mathrm{P}<0.001)$. This suggests that the CCMV-ASNase does consist of active tetramers and that the encapsulation imposes an improvement in this effect over the free enzyme. However, it requires further study to definitively support this hypothesis.

\subsubsection{Discussion}

The change in effective ALL cell death cannot be explained solely by the difference in enzyme activity as measured by NMR. These measurements showed a $2.5 \cdot 10^{4}$ and 8 times lower ASNase activity in encapsulin and CCMV, respectively, compared to the unmodified enzyme. When compared to the free ASNase, the induction of cell death is $10^{3}$ times lower for BlE-ASNase and is the same with CCMV-ASNase. Figure 4.8 shows that BlE and CCMV subunits without the enzyme are not toxic at the used concentrations and the observed reduced cell viability is therefore only the result of ASNase activity (green in Figure 4.8B, grey in Figure $4.8 \mathrm{C})$. 
A possible reason for this discrepancy is the increased stability of the enzyme in either protein nanocage. The NMR experiments were done in $\mathrm{D}_{2} \mathrm{O}$, but the cell experiments were done in culture medium. This culture medium can affect free proteins and the ASNase can therefore be degraded by both proteases that are present in the culture medium and by the binding of medium components to the enzyme, which can induce conformational changes of the ASNase or block the active site, for example by inhibitors. ${ }^{40,41}$ As intended, the protein cages can form a protective shell and thus prevent the inactivation of the ASNase, which was also observed in literature for an unstable enzyme in a bacteriophage. ${ }^{19}$

While with cryo-EM we were able to analyse the structure of both BlE and CCMV, the exact structural conformation of the ASNase cargo in these cages remains unknown. For CCMV-ASNase, we added ASNase modified with PSS to CCMV capsid proteins and these assembled into particles, with one ASNase tetramer per particle based on the cryo-EM data. $^{28}$ There is no further structural modification of the enzymes and they are already correctly folded before addition to CCMV capsid protein. We used the same non-encapsulated ASNase with and without the PSS and they both showed high activity. The data suggest that the PSS modification does not affect the ASNase structure.

For the BlE-ASNase, the tetramer formation and encapsulin loading happens in the bacteria and can therefore not be controlled. The exact folding and tetramer formation of the ASNase in BlE is thus unknown. With cryo-EM we estimated the volume in the encapsulin cage that was filled with ASNase cargo, which matched $\sim 1$ tetramer. However, these data are not conclusive and require additional study. Because of the different orientations of the ASNase cargo in the encapsulin, it is difficult to obtain a high resolution map of the cargo of the encapsulin. 
With SDS-PAGE we studied the number of ASNase monomers per encapsulin cage (Figure 4.2B), but the amount of monomers does not necessarily correlate to correctly folded active tetramers. However, this does not undermine the conclusion that the protein shell is an important barrier for substrate and product crossing. If $\mathrm{BlE}$ on average contains more than one active ASNase tetramer (SDS-PAGE data suggest 15 monomers (Figure $4.2 \mathrm{C})$ ), the induction of cell death is still 1,000x lower than CCMV which contains one active ASNase tetramer. This is probably caused by the differences in the diffusion of substrate and product into and out of the protein shell. The most likely hypothesis is that both the influx and efflux of L-asparagine and L-aspartate into and out of the encapsulin are limited, but the low efflux of L-aspartate causes product inhibition.

$\mathrm{BlE}$ nanoparticles are resistant to degradation caused by changes in $\mathrm{pH}$ or salt concentration. ${ }^{33}$ While in this setting CCMV-ASNase induces ALL cell death at a 1000x lower concentration compared with BlE-ASNase, in other settings the stability provided by BlE could provide a benefit over other protein nanocages. Thus, it is important to study different protein nanocages depending on the application.

\subsection{Conclusions}

We successfully encapsulated ASNase in both CCMV and Bl encapsulin. We show that ASNase, when encapsulated, remains active. Depending on the choice of nanoparticle, however, the activity of ASNase can be similar to that of the native enzyme, or significantly altered. The cage porosity, not only determined by the channel diameters, but also by the distribution of channel charges, is a major factor to be evaluated. This principle can be used to create monodisperse nanoreactors that contain enzymes with specific activity. This is also seen in the cell experiments, in which ASNase encapsulated in CCMV shows activity in inducing ALL cell death 
similar or even better to that of the native ASNase, while a 1,000x higher concentration is required when encapsulated in BlE. Overall, we show that the encapsulation of ASNase in protein nanocages holds potential for combining enzyme therapy with the advantages of protein cages.

\subsection{Acknowledgements}

I thank dr. M. V. de Ruiter for his assistance with the parts related to CCMV and V. M. Dombrowe for her work on the cell studies. I thank Prof. dr. J. R. Castón and his colleagues for all the fantastic work on the cryo-EM analyses. I also thank dr. Alberto Juan Ruiz Del Valle for his help with the NMR measurements.

\subsection{Experimental Section}

\subsubsection{Materials}

Buffers were prepared using double deionized water from a Millipore system. Plasmids containing BlE with green fluorescent protein (GFP) cargo were kindly provided by dr. Rurup. The encapsulin plasmids were cultured in E. coli Nova Blue and expressed in E. coli Rosetta Gami (DE3). CCMV was purified from the cowpea plant and its native genetic cargo was removed to acquire the free capsid protein dimers (CP) according to literature procedures. ${ }^{42}$ All reactions were carried out at $20^{\circ} \mathrm{C}$ and proteins and assemblies were stored at $4^{\circ} \mathrm{C}$. All other materials were obtained from Sigma-Aldrich unless stated otherwise. 


\subsubsection{Fabrication and Encapsulation of Negatively Charged ASNase in Virus-like Particles}

The enzyme loaded VLP complex was carried out similarly to literature procedures, ${ }^{28,43}$ albeit with a different enzyme. To get negatively charged ASNase, the enzyme was reacted to sulfo-EMCS $(40 \mu \mathrm{M})$ (Thermo Fisher) in phosphate buffered saline (PBS). This was purified using ZEBA 7k MWCO columns (Thermo Fisher) and subsequently reacted with an excess of thiolated polystyrene sulfonate (PSS-SH) polymers of $20 \mathrm{kDa}$, which were pre-incubated with an excess of tris(2-carboxyethyl)phosphine (TCEP) to reduce the disulfide bonds. This was purified by SEC with a GE Healthcare FPLC Äkta purifier 900 combined with a Frac 950 fraction collector. We used a $10 \mathrm{mM} \mathrm{NaCl}$, Tris $\mathrm{HCl}$ buffer (pH 7). Samples were purified over a preparative column superose 6 10/100 GL (GE Healthcare) with a $24 \mathrm{~mL}$ bed volume. The purified ASNase-PSS complex was encapsulated by mixing this with free $\mathrm{CP}$ in a 1:180 ratio, in assembly buffer (50 mM Tris, $100 \mathrm{mM} \mathrm{NaCl}, 10 \mathrm{mM} \mathrm{KCl}$ and $5 \mathrm{mM} \mathrm{MgCl} 2 \mathrm{pH} 7.2$ ) and incubating overnight at $4{ }^{\circ} \mathrm{C}$. The formed VLPs were again purified using SEC.

\subsubsection{Production and Purification of BlE-ASNase}

We used the plasmid DNA from BlE with GFP cargo. This contained an operon of GFP (with the spacer and affinity sequence that links the cargo protein to the capsid proteins) and encapsulin genes. The gene coding for GFP, which contained restriction sites at the beginning (XbaI) and end before the spacer and affinity sequence (XmaI), was removed using restriction enzymes. The gene coding for ASNase (Eurofins) was inserted by ligation. The plasmid was inserted in E. coli Nova Blue bacteria using heat shock membrane permeabilization and cultured to enhance plasmid production. The plasmids were extracted from the E. coli Nova Blue cells using QIAprep Spin Miniprep Kit (Qiagen) and inserted into E. 
coli Rosetta Gami DE3 bacteria. These bacteria were cultured and the encapsulins were extracted according to a protocol based on work by Rurup et al., ${ }^{35,36}$ which is detailed in Chapter 3.

\subsubsection{Dynamic Light Scattering}

We performed DLS using a Nanotrac wave W3043 from Microtrac. Data were extracted using the company's software. The solution viscosity and refractive index of water were used, as well as a refractive index of 1.54 for the sample.

\subsubsection{Transmission Electron Microscopy}

For conventional electron microscopy, $5 \mu \mathrm{L}$ samples were applied to glow discharged carbon-coated grids and negatively stained with $2 \%(\mathrm{v} / \mathrm{v})$ aqueous uranyl acetate. Images were acquired on a HEOL JEM-1011 electron microscope (JEOL Ltd., Tokyo, Japan) operated at $100 \mathrm{kV}$, with a 4K·2.7K ES1000W Erlangshen CCD camera (Gatan, Warrendale, PA, USA) at a nominal magnification of 24,000x.

\subsubsection{SDS-PAGE}

We performed SDS-PAGE on precast stain free 4-15\% gels from BioRad. Known concentrations of the enzyme, BlE and CCMV were used to estimate the encapsulation efficiency. A Bio-Rad protein precision plus ladder was used as a reference. The SDS-PAGE was run on $200 \mathrm{~V}$ for 0.5 $\mathrm{h}$ with a denatured $10 \mu \mathrm{l}$ sample mixed with $10 \mu \mathrm{l}$ gel loading dye (BioRad) with $10 \%(\mathrm{v} / \mathrm{v}) \beta$-mercaptoethanol. SDS-PAGE data were analysed using image lab software (Bio-Rad). 
For additional purification the BlE-ASNase sample was re-purified by ultracentrifugation through a $20-50 \%$ (w/v) linear sucrose gradient $(273,620$ $\mathrm{x}$ g, $1 \mathrm{~h} 15 \mathrm{~min}$ ). The gradient was prepared in $20 \mathrm{mM}$ Tris- $\mathrm{HCl}, 150 \mathrm{mM}$ $\mathrm{NH}_{4} \mathrm{Cl}$, pH 7.5 buffer. Fractions $(1 \mathrm{ml})$ were concentrated 10 -fold by ultracentrifugation $(302,986 \mathrm{xg}, 2 \mathrm{~h})$. All purification steps were performed at $4{ }^{\circ} \mathrm{C}$. We collected fractions of the sucrose gradient and performed an SDS-PAGE. Fraction 10 was used for cryo-EM.

The intensity of both proteins bands from fraction 10 were measured using a Quantity One volume measure tool. The background signal to the intensity measures was subtracted, thus resulting in a final normalized bands intensity quantification. These quantification values were assumed to be equivalent to $\sim$ grams of proteins $(2,268$ for encapsulin and 766 for ASNase). BlE and ASNase moles were estimated dividing grams by their molecular weight $(2,268 \sim$ grams $/ 32 \mathrm{kDa})$ for $\mathrm{BlE}$ and $(766 \sim$ grams $/ 40$ $\mathrm{kDa}$ ) for ASNase. This resulted $\sim$ moles $=71$ for BlE and 19 for ASNase.

To calculate the molar ratio between these proteins the BlE moles were divided by ASNase moles, resulting in a 3.75:1 ratio. This means that per one ASNase there are $\sim 4$ copies of BlE monomer. This means that up to 15 copies of ASNase monomers are internalized inside of the $\mathrm{T}=1$ icosahedral capsid (a $\mathrm{T}=1$ capsid is formed by 60 copies of the coat $\mathrm{BlE}$ protein). In other words, a maximum of three ASNase tetramers would be encapsulated inside BlE.

\subsubsection{ELISA}

The ELISA was performed by incubating the samples at a concentration between 10 and $0 \mathrm{nM}$ on ELISA plates overnight, followed by $3 \mathrm{x}$ washing with 1x PBS ( $\mathrm{pH} 7$ ) and blocking of the unreacted groups of the plate with $10 \%$ bovine serum albumin. The plate was incubated overnight with 10,000x diluted rabbit IgG antibody for ASNase from Abcam and washed 
with PBS (as before). A Rabbit IgG Elisa kit from Abcam was used according to the protocol of that kit. Absorbance was measured at $\lambda=$ $450 \mathrm{~nm}$ on a Tecan i-control infinite 200 Pro as an endpoint measurement.

\subsubsection{Cryo-EM and Image Processing}

BlE-ASNase or CCMV-ASNase samples $(5 \mu \mathrm{L})$ were applied to R2/2 300 mesh copper-rhodium grids (Quantifoil Micro Tools, Germany) and vitrified using Leica Automatic Plunge Freezer. Data were collected on a FEI Talos Arctica electron microscope operating at $200 \mathrm{kV}$ and images recorded on a FEI Falcon III detector at a nominal magnification of 73,000 yielding a pixel size of $1.42 \AA$. A total dose of 30 electrons $/ \AA^{2}$ was used to record 148 (for BlE-ASNase) or 3,786 (for CCMV-ASNase) 40-frame movies with a defocus range of 1.0 to $3.3 \mu \mathrm{m}$.

The frames of each movie were motion corrected, dose-weighted and summed with the MotionCor2 frame alignment program. ${ }^{44}$ Contrast transfer function parameters were determined with CTFFIND $4^{45}$ using unweighted summed images. Images showing a stigmatism and/or motion signs were discarded, maintaining a total of 137 and 3,369 for BlE-ASNase and CCMV-ASNase, respectively. General image processing operations were performed using Xmipp ${ }^{46}$ and Relion. ${ }^{47}$ Graphics were generated by UCSF Chimera. ${ }^{48}$ Particles were picked with the Xmipp automatic picking routine. Relion reference-free $2 \mathrm{D}$ classification was used to discard bad particles, resulting in data sets of 9,548 and 383,770 particles for BlE and CCMV, respectively.

3D classification imposing icosahedral symmetry (I2) with Relion resulted in three classes using as a reference volume a model obtained with the EMAN2 initial model routine, ${ }^{49}$ or the map of Zn-Pc loaded CCMV T = 1 capsid. ${ }^{37}$ The best classes (6,251 particles for BlE-ASNase and 237,489 particles for CCMV-ASNase) were iteratively auto-refined using Relion. 
The resolution reached was $4.48 \AA$ for BlE-ASNase and $5.58 \AA$ for CCMVASNase. The best BlE-ASNase class was further 3D classified without imposing icosahedral symmetry $(\mathrm{C} 1)$ and the resolution was $11.83 \AA$. The resolutions were based on the gold standard Fourier shell correlation (FSC) $\mathrm{FSC}=0.143$ criterion corrected for the effects of a soft mask on the FSC curve using high-resolution noise substitution. The resolutions were obtained using the complete data set of 3D-selected particles. The final density map was corrected for the modulation transfer function of the detector and sharpened by applying the estimated B-factor.

The Chimera fitting tool was used to dock the atomic crystallographic model of Thermotoga maritima encapsulin (PDB entry 3DKT) into our cryo-EM encapsulin maps. Monomer corresponding to the chain A from the $\mathrm{T}=3$ capsid of CCMV (PDB entry 1CWP) was first fitted as a rigid body using UCSF Chimera. Then, the C-terminus (residues from 179 to 190) was docked as a jelly body using Coot version $0.8 .7^{50}$ to place them in its appropriate density of the cryo-EM map.

\subsubsection{Data Deposition}

The I2 BlE-ASNase and CCMV-ASNase cryo-EM maps are deposited in the Electron Microscopy Data Bank with accession numbers EMD-4606 and EMD-4607, respectively. 


\subsubsection{Activity of Encapsulated ASNase}

To prepare the samples for NMR, ASNase and the complexes were dissolved and diluted in $\mathrm{D}_{2} \mathrm{O}$ with $0.05 \%$ wt. 3 (trimethylsilyl)propionic2,2,3,3-d4-acid, sodium salt (TSP). Residual $\mathrm{H}_{2} \mathrm{O}$ and buffer constituents were removed using ZebaSpin columns with an MWCO of $7 \mathrm{kDa}$. Lasparagine was added and the L-asparagine depletion and L-aspartate formation were analysed over time with NMR. All NMR experiments were carried out in a Bruker Avance II $600 \mathrm{MHz}$ spectrometer (14.1 T) provided with a $5 \mathrm{~mm}$ triple-nucleus (TXI) probe head with z-gradients coil with maximum gradient strength of $50 \mathrm{G} / \mathrm{cm}^{-1}$. Chemical shifts are reported in ppm and referenced to TSP. ${ }^{1} \mathrm{H}$ NMR water suppression pulse sequence with excitation sculpting and gradients from Bruker pulse program library was applied. The following NMR acquisition parameters were applied: 64 scans, $1 \mathrm{sec}$ delay time and $1.7 \mathrm{sec}$ of acquisition time.

With the software Mnova (Mestrelab), the NMR data was processed. With Mnova integrals were calculated and used to determine the concentrations of L-asparagine and L-aspartate, given the known starting concentration and corresponding peak integral. During the reaction, the integrals of the L-asparagine and L-aspartate peaks were added and the percentage of each compound was calculated.

\subsubsection{Induction of ALL Cell Death by Encapsu- lated ASNase}

To determine the induction of cell death by ASNase (either encapsulated or not), we cultured the human ALL cell line SUP-B15 (ACTT) according to the ACTT-CRL 1929 protocol. We used Iscove's Modified Dulbecco's Medium (IMDM) that contained $4 \mathrm{mM}$ L-glutamine, $4.5 \mathrm{~g} / \mathrm{L}$ glucose and $1.5 \mathrm{~g} / \mathrm{L} \mathrm{NaH}_{2} \mathrm{CO}_{3}$ which was supplemented with $20 \%$ (v/v) fetal bovine serum (FBS), $100 \mathrm{U}$ streptomycin and 0.1 mgŁpenicillin. 
Cells were seeded at 100,000 cells per well in a Greiner 96 well plate. To these cells we added the ASNase in various formulations and concentrations in a 1:10 (v/v) ratio. Cells were cultured at $37^{\circ} \mathrm{C}, 5 \% \mathrm{CO}_{2}$ for 24 , 48 and $72 \mathrm{~h}$, after which the samples were centrifuged at 125x g for 5 min in a $15 \mathrm{~mL}$ tube (Eppendorf Centrifuge 5702). The cell pellets were resuspended in PBS. With a FACS ARIA II (BD Biosciences), 10,000 counts per sample were analysed. Just prior to the measurement, propidium iodide was added to each sample to differentiate between alive and dead cells, on which we based our cell viability percentage.

\subsubsection{Statistics}

The statistical analysis was performed using R 3.4.3 software. We used a univariate ANOVA derived linear model to determine the statistical differences between different concentrations within one treatment. Using a t-test with pooled standard deviations, we calculated the significance between treatments. 


\subsection{References}

1. Pieters, R.; Hunger, S. P.; Boos, J.; Rizzari, C.; Silverman, L.; Baruchel, A.; Goekbuget, N.; Schrappe, M.; Pui, C. L-asparaginase Treatment in Acute Lymphoblastic Leukemia: a Focus on Erwinia Asparaginase. Cancer. 2011, 117, 238-249

2. $\quad$ Egler, R. A.; Ahuja, S. P.; Matloub, Y. L-asparaginase in the Treatment of Patients with Acute Lymphoblastic Leukemia. J. Pharmacol. Pharmacother. 2016, 7, 62-71

3. Novak, E. K.; Phillips, A. W. L-glutamine as a Substrate forLasparaginase from Serratia Marcescens. J. Bacteriol. 1974, 117, 593-60 4. Hermanova, I.; Zalinova, M.; Trka, J.; Starkova, J. Low Expression of Asparagine Synthetase in Lymphoid Blasts Precludes its Role in Sensitivity toL-asparaginase. Exp. Hematol. 2012, 40, 657-665

5. Su, N.; Pan, Y.; Zhou, M.; Harvey, R. C.; Hunger, S. P.; Kilberg, M. S. Correlation between Asparagine Sensitivity and Asparagine Synthetase Protein Content, but not mRNA, in Acute Lymphoblastic Leukemia Cell Lines. Pediatr. Blood Cancer. 2008, 50, 274-279

6. Asselin, B. L.; Ryan, D.; Frantz, C. N.; Bernal, S. D.; Leavitt, P.; Sallan, S. E.; Cohen, H. J. In vitro and in Vivo Killing of Acute Lymphoblastic Leukemia Cells by L-asparaginase. Cancer Res. 1989, 49, 4363-4368

7. Hawkins, D. S.; Park, J. R.; Thomson, B. G.; Felgenhauer, J. L.; Holcenberg, J. S.; Panosyan, E. H.; Avramis, V. I. Asparaginase Pharmacokinetics after Intensive Polyethylene Glycol-conjugated L-asparaginase Therapy for Children with Relapsed Acute Lymphoblastic Leukemia. Clin. Cancer Res. 2004, 10, 5335-5341

8. Hijiya, N.; van der Sluis, I. M. Asparaginase-associated Toxicity in Children with Acute Lymphoblastic Leukemia. Leuk. Lymphoma. 2016, $57,748-757$ 
9. Andrade, A. F.; Borges, K. S.; Silveira, V. S. Update on the Use of L-asparaginase in Infants and Adolescent Patients with Acute Lymphoblastic Leukemia. Clin. Med. Insights Oncol. 2014, 8, 95-100

10. Bhaskar, S.; Lim, S. Engineering Protein Nanocages as Carriers for Biomedical Applications. NPG Asia Mat. 2017, 9, e371

11. Schoonen, L.; van Hest, J. C. Functionalization of Protein-based Nanocages for Drug Delivery. Nanoscale. 2014, 6, 7124-7141

12. Bae, Y.; Kim, G. J.; Kim, H.; Park, S. G.; Jung, H. S.; Kang, S. Engineering Tunable Dual Functional Protein Cage Nanoparticles Using Bacterial Superglue. Biomacromolecules, 2018, 19, 2896-2904

13. Azuma, Y.; Bader, D. L. V.; Hilvert, D. Substrate Sorting by a Supercharged Nanoreactor. J. Am. Chem. Soc. 2018, 140, 860-863 14. Liu, A.; Traulsen, C. H.-H.; Cornelissen, J. J. L. M. Nitroarene Reduction by a Virus Protein Cage Based Nanoreactor. ACS Catal. 2016, 6, 3084-3091

15. Pitek, A. S.; Jameson, S. A.; Veliz, F. A.; Shukla, S.; Steinmetz, N. F. Serum Albumin 'Camouflage' of Plant Virus Based Nanoparticles Prevents their Antibody Recognition and Enhances Pharmacokinetics. Biomaterials. 2016, 89, 89-97

16. Steinmetz, N. F.; Manchester, M. PEGylated Viral Nanoparticles (VNPs) for Biomedicine: the Impact of PEG Chain Length on VNP Cell Interactions In Vitro and Ex Vivo. Biomacromolecules 2009, 10, 784-792 17. Moon, H.; Lee, J.; Kim, H.; Heo, S.; Min, H.; Kang, S. Genetically Engineering Encapsulin Protein Cage Nanoparticle as a SSC-7 Cell Targeting Optical Nanoprobe. Biomaster. Res. 2014, 18, 21

18. Koyani, R.; Pérez-Robles, J.; Cadena-Nava, R. D.; Vazquez-Duhalt, R. Biomaterial-based Nanoreactors, and Alternative for Enzyme Delivery. Nanotech. Rev. 2017, 6, 405-419

19. O’Neil, A.; Prevelige, P. E.; Douglas, T. Stabilizing Viral Nanoreactors for Nerve-agent Degradation. Biomater. Sci. 2013, 1, 881-886 
20. Jordan, P. C.; Patterson, D. P.; Saboda, K. N.; Edwards, E. J.; Miettinen, H. M.; Basu, G.; Thielges, M. C.; Douglas, T. Self-assembling Biomolecular Catalysts for Hydrogen Production. Nat. Chem. 2016, 8, 179-185

21. Wörsdörfer, B.; Woycechowsky, K. J.; Hilvert, D. Directed Evolution of a Protein Container. Science. 2011, 331, 589-592

22. Tetter, S.; Hilvert, D. Enzyme Encapsulation by a Ferritin Cage. Angew. Chem. Int. Ed. Engl. 2017, 56, 14933-14936

23. Tagit, O.; de Ruiter, M. V.; Brasch, M.; Ma, Y.; Cornelissen, J. J. L. M. Quantum Dot Encapsulation in Virus-like Particles with Tuneable Structural Properties and Low Toxicity. RSC Adv. 2017, 60, 3811038118

24. Caspar, D. L.; Klug, A. Physical Principles in the Construction of Regular Viruses. Cold Spring Harb. Symp. Quant. Biol. 1962, 27, 1-24 25. Bancroft, J. B.; Hiebert, E. Formation of an Infectious Nucleoprotein from Protein and Nucleic Acid Isolated from a Small Spherical Virus. Virology. 1967, 32, 354-356

26. Lavelle, L.; Michel, J. P.; Gingery, M. The Disassembly, Reassembly and Stability of CCMV Protein Capsids. J. Virol. Methods. 2007, $146,311-316$

27. Liepold, L. O.; Revis, J.; Allen, M.; Oltrogge, L.; Young, M.; Douglas, T. Structural Transitions in Cowpea Chlorotic Mottle Virus (CCMV). Phys. Biol. 2005, 2, S166-S172

28. Brasch, M.; Putri, R. M.; de Ruiter, M. V.; Luque, D.; Koay, M. S.; Castón, J. R.; Cornelissen, J. J.; Assembling Enzymatic Cascade Pathways inside Virus-based Nanocages Using Dual-tasking Nucleic Acid Tags. J. Am. Chem. Soc. 2017, 139, 1512-1519

29. Comellas-Aragonès, M.; Engelkamp, H.; Claessen, V. I.; Sommerdijk, N. A.; Rowan, A. E.; Christianen, P. C.; Maan, J. C.; Verduin, B. J.; Cornelissen, J. J.; Nolte, R. J. A Virus-based Single-enzyme Nanoreactor. Nat. Nanotechnol. 2007, 2, 635-639 
30. Sánchez-Sánchez, L.; Tapia-Moreno, A.; Juarez-Moreno, K.; Patterson, D. P.; Cadena-Nava, R. D.; Douglas, T.; Vazquez-Duhalt, R.; Design of a VLP-nanovehicle for CYP450 Enzymatic Activity Delivery. J. Nanobiotechnology 2015, 13, 66

31. Sutter, M.; Boehringer, D.; Gutmann, S.; Günther, S.; Prangishvili, D.; Loessner, M. J.; Stetter, K. O.; Weber-Ban, E.; Ban, N. Structural Basis of Enzyme Encapsulation into a Bacterial Nanocompartment. Nat. Struct. Mol. Biol. 2008, 15, 939-947

32. Giessen, T. W.; Silver, P. A. Widespread Distribution of Encapsulin Nanocompartments Reveals Functional Diversity. Nat. Microbiol. 2017, 6, 17029

33. Putri, R. M.; Allende-Ballestero, C.; Luque, D.; Klem, R.; Rousou, K. A.; Liu, A.; Traulsen, C. H.; Rurup, W. F.; Koay, M. S. T.; Castón, J. R.; Cornelissen, J. J. L. M. Structural Characterization of Native and Modified Encapsulins as Nanoplatforms for in Vitro Catalysis and Cellular Uptake. ACS Nano, 2017, 11, 12796-12804

34. Lagoutte, P.; Mignon, C.; Stadthagen, G.; Potisopon, S.; Donnat, S.; Mast, J.; Lugari, A.; Werle, B. Simultaneous Surface Display and Cargo Loading of Encapsulin Nanocompartments and their use for Rational Vaccine Design. Vaccine, 2018, 36, 3622-3628

35. Rurup, W. F.; Snijder, J.; Koay, M. S.; Heck, A. J.; Cornelissen, J. J. Self-sorting of Foreign Proteins in a Bacterial Nanocompartment. J. Am. Chem. Soc. 2014, 136, 3828-3832

36. Rurup, W. F.; Cornelissen, J. J. L. M.; Koay, M. S. T. Recombinant Expression and Purification of 'Virus-like' Bacterial Encapsulin Protein Cages. In B. P. Orner (ed.), Protein Cages: Methods in Molecular Biology (Methods and Protocols), Vol. 1252, 61-67 New York: Humana Press. 
37. Luque, D.; de la Escosura, A.; Snijder, J.; Brasch, M.; Burnley, R, J,; Koay, M. S. T.; Carrascosa, J. L.; Wuite, G. J. L.; Roos, W. H.; Heck, A. J. R.; Cornelissen, J. J. L. M.; Torres, T.; Castón, J. R. Self-assembly and Characterization of Small and Monodisperse Dye Nanospheres in a Protein Cage. Chem. Sci. 2014, 5, 575-581

38. Stephanopoulos, N.; Francis, M. B. Choosing and Effective Protein Bioconjugation Strategy. Nat. Chem. Biol. 2011, 7, 876-884

39. Wilts, B. D.; Schaap, I. A. T.; Schmidt, C. F. Swelling and Softening of the Cowpea Chlorotic Mottle Virus in Response to pH Shifts. Biophys. J. 2015, 108, 2541-2549

40. Sanfins, E.; Augustsson, C.; Dahlbäck, B.; Linse, S.; Cedervall, T. Size-dependent Effects of Nanoparticles on Enzymes in the Blood Coagulation Cascade. Nano Lett. 2014, 14, 4736-4744

41. Iyer, P. V.; Ananthanarayan, L. Enzyme Stability and Stabilization - Aqueous and Non-aqueous Environment. Process Biochem. 2008, 43, 1019-1032

42. Sikkema, F. D.; Comellas-Aragonès, M.; Fokkink, R. G.; Verduin, B. J. M.; Cornelissen, J. J. L. M.; Nolte, R. J. M., Monodisperse polymervirus hybrid nanoparticles. Organic and Biomolecular Chemistry 2007, $5,54-57$

43. Liu, A.; de Ruiter, M. V.; Zhu, W.; Maassen, S. J.; Yang, L.; Cornelissen, J. J. L. M. Compartmentalized Thin Films with Customized Functionality via Interfacial Cross-linking of Protein Cages. Adv. Func. Mat. 2018, 28, 1801574

44. Li, X.; Mooney, P.; Zheng, S.; Booth, C. R.; Braunfeld, M. B.; Gubbens, S.; Agard, D. A.; Cheng, Y. Electron Counting and Beam Induced Motion Correction Enable Near-atomic-resolution Single-particle Cryo-EM. Nat. Methods. 2013, 10, 584-90

45. Rohou, A.; Grigorieff, N.; CTFFIND4: Fast and Accurate Defocus Estimation from Electron Micrographs. J. Struct. Biol. 2015, 192, 216-21 
46. De la Rosa-Trevín, J. M.; Oton, J.; Marabini, R.; Zaldivar, A.; Vargas, J.; Carazo, J. M.; Sorzano, C. O. Xmipp 3.0: an Improved Software Suite for Image Processing in Electron Microscopy. J. Struct. Biol. 2013, 184, 321-8

47. Scheres, S. H. W. RELION: Implementation of a Bayesian Approach to Cryo-EM Structure Determination. J. Struct. Biol. 2012, 180,51930

48. Pettersen, E. F.; Goddard, T. D.; Huang, C. C.; Couch, G. S.; Greenblatt, D. M.; Meng, E. C.; Ferrin, T. E. UCSF Chimera - a Visualization System for Exploratory Research and Analysis. J. Comput. Chem. 2004, 25, 1605-12

49. Tang, G.; Peng, L.; Baldwin, P. R.; Mann, D. S.; Jiang, W.; Rees, I.; Ludtke, S. J. EMAN2: an Extensible Image Processing Suite for Electron Microscopy. J. Struct. Biol. 2007, 157, 38-46

50. Emsley, P; Cowtan, K. Coot: model-building tools for molecular graphics. Acta Crystallogr. D. Biol. Crystallogr. 2004, 60, 2126-32 
Construction and Evaluation of L-Asparaginase loaded Protein Cages 
Chapter 4 


\section{Chapter 5}

\section{Uptake, Retention and Immunogenicity of}

Brevibacterium linens

\section{Encapsulin in Cells}

\section{Abstract}

While encapsulins could be useful as a vessel for enzymes to treat various diseases or as a vaccine carrier, the effects of encapsulins on cells and that of cells on encapsulins remain unknown. We analyse the uptake and processing of Brevibacterium linens encapsulin and its cargo (monomeric teal fluorescent protein) in various cell types and study the immunogenicity of these encapsulins in RAW264.7 and J774 macrophages. Increasing the understanding of the interaction between encapsulin and cells allows for more specific modifications to the encapsulin to apply them in therapeutic applications. 


\subsection{Introduction}

Nanoparticles can be used to transport cargo, such as proteins, small molecules and genes, to cells. However, different nanoparticles have different uptake routes in cells since many aspects influence the internalization. ${ }^{1}$ The uptake route determines the fate of the nanoparticle and its cargo after it enters the cell. Particles that end up in endosomes/lysosomes are more prone to degradation than particles that enter the cell via active membrane transport and end up in the cytoplasm. ${ }^{2,3}$ For potential medical use, it is important to know the fate of nanoparticles in the cells and how this can be modulated. The more detailed the knowledge on uptake and processing of nanoparticles in cells, the more specific a potential modification can be made. In this chapter, we study the uptake of encapsulins in various cells.

Encapsulins are bacterial protein nanoparticles in the size range of 20 - $40 \mathrm{~nm} .{ }^{4}$ Their structure often adheres to a $\mathrm{T}=1$ or $\mathrm{T}=3$ icosahedral symmetry and the structure consists of 60 or 180 protein monomers that form the protein nanoparticle. In this work we use encapsulins derived from Brevibacterium linens (BlE). While BlE naturally contains a dye decolorizing peroxidase, we used BlE modified to contain monomeric teal fluorescent protein (mTFP) cargo for easier detection in cell studies. ${ }^{5}$

The cellular uptake mechanism of encapsulins has not been studied yet, but it is important to do so, because the uptake route determines the fate of the nanoparticles after entering the cell. ${ }^{1}$ When transporting enzymes, the cargo has to pass the cell membrane but ideally does not end up in lysosomal structures. When transporting genes, the cargo has to end up in the nucleus, which requires it to pass the nucleic envelope as well. Finding out the native uptake of encapsulins provides insight into which modifications are required for optimal encapsulin uptake, but also for prolonged encapsulin retention at a target site. 
Furthermore, since encapsulins are nanoparticles, they are more likely to end up in immune cells such as macrophages when administered to people compared to smaller molecules or non-foreign proteins. ${ }^{6}$ This means that they are more likely to be broken down in the macrophages and trigger an immune response. ${ }^{7}$ The latter is also more likely to occur given that encapsulins are bacterial in origin. Therefore, we studied the immunological response of encapsulins. The uptake of encapsulins in macrophages is important in two ways: when encapsulins are used as enzyme carrier the immune response is a side effect that should be prevented. On the other hand when encapsulins are used as vaccine carrier they might benefit from a stronger immune response. ${ }^{8}$

In this chapter we study the uptake of encapsulins in different cells and the mechanism thereof. We predominantly focus on macrophages to study the potential immune response triggered by encapsulin administration as well. This study develops a method which could be of great advantage when exploring potential modifications to enhance encapsulin properties for targeted drug delivery.

\subsection{Results and Discussion}

\subsubsection{Uptake of Brevibacterium linens Encapsulins in Various Cells}

To study the processing of encapsulins in cells, we loaded BlE with mTFP (BlE-mTFP). The emission of this fluorescent protein can be analysed at $\lambda_{\mathrm{em}}=530 / 30 \mathrm{~nm}$ when excited at $\lambda_{\mathrm{ex}}=460 \mathrm{~nm}$. First, we studied the time it takes for encapsulins to be taken in HeLa cancer cells using 2(4-Amidinophenyl)-1H-indole-6-carboxamidine (DAPI) as marker for the nucleus and Wheat Germ Agglutinin (WGA) for the cell membrane. 

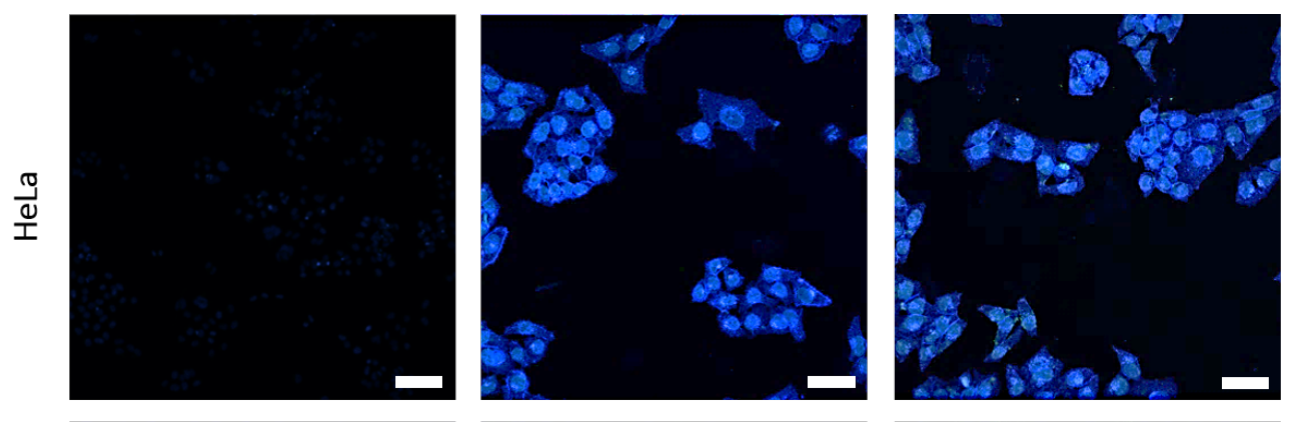

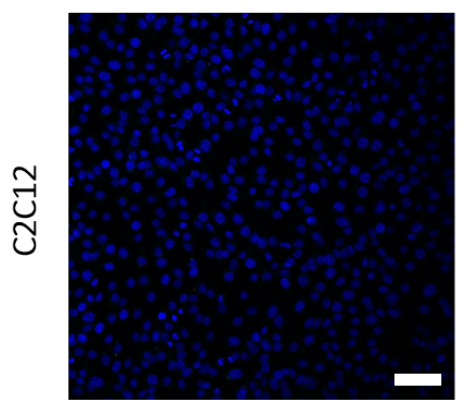

Negative Control

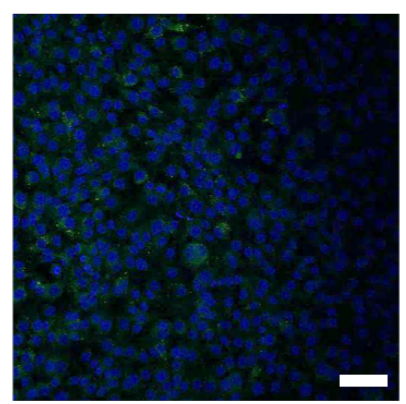

500 nM BIE-mTFP $4 \mathrm{~h}$

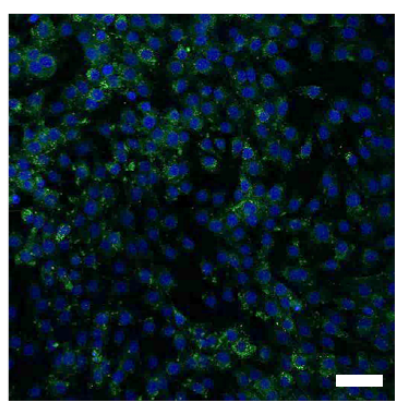

$500 \mathrm{nM}$ BIE-mTFP $24 \mathrm{~h}$

Figure 5.1: Confocal microscopy images of the uptake of BlE-mTFP in HeLa and C2C12 cells. The blue signal corresponds to DAPI (cell nuclei) and WGA (cell membranes), the green signal corresponds to the mTFP. Scalebars represent $20 \mu \mathrm{m}$ for HeLa and $50 \mu \mathrm{m}$ for C2C12 images.

In these HeLa cells we found no uptake of encapsulins. We studied the uptake of BlE-mTFP in HeLa cells after incubating up to $24 \mathrm{~h}$, with different concentrations of BlE-mTFP $(0-500 \mathrm{nM})$, based on previous uptake studies in J774 cells. ${ }^{5}$ After incubation we removed the medium containing the BlE-mTFP and fixed the cells (Figure 5.1, top row). Since other protein nanoparticles are internalized by HeLa cells, ${ }^{9}$ this outcome was unexpected. It is unlikely that the mTFP is broken down that rapidly, meaning that the lack of signal is likely due to the lack of uptake of BlEmTFP. Hence, we chose a different non-macrophage cell line, $\mathrm{C} 2 \mathrm{C} 12$, to study the uptake. In the $\mathrm{C} 2 \mathrm{C} 12$ cells, which are mouse myoblast cells, we found minor uptake after $4 \mathrm{~h}$ and $24 \mathrm{~h}$ (Figure 5.1, bottom row). 


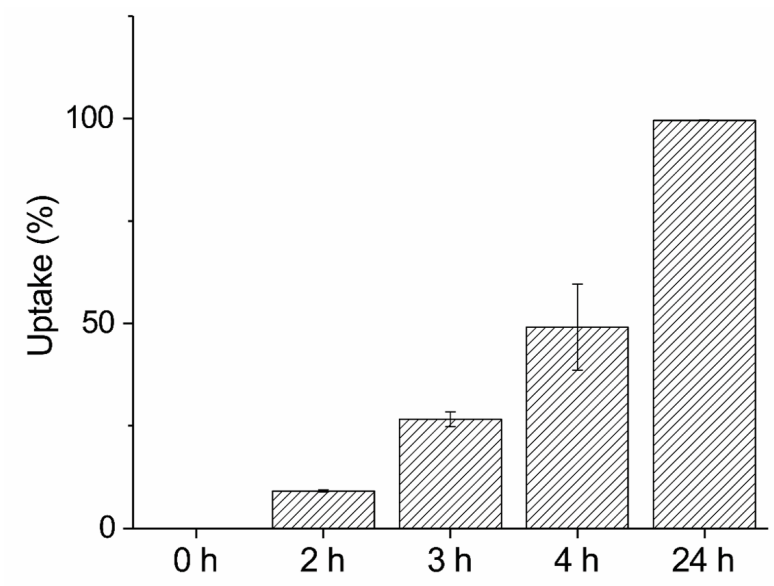

Figure 5.2: The uptake of BlE-mTFP in RAW264.7 macrophages as determined by flow cytometry. The $\mathrm{y}$-axis corresponds to the percentage of cells that had mTFP taken up to a limit detectable by flow cytometry. In the $24 \mathrm{~h}$ sample, the morphology of the macrophages changed, for which the flow cytometry dot plot gating strategy had to be changed. The error bars represent the standard deviation $(\mathrm{N}=3)$.

The low uptake in cells is in line with research done by Moon et al., ${ }^{10}$ who also found no uptake of encapsulins in various cell lines (HeLa, HepG2, $\mathrm{KB}$ and MDAMB231) unless they added targeting moieties on the exterior. We chose the RAW264.7 macrophage cell line to study the uptake, even though the uptake mechanisms are likely to be different compared with non-macrophage cells like HeLa and C2C12. ${ }^{11}$

We found that the encapsulins are taken up slowly in the RAW264.7 cells (Figure 5.2). After $24 \mathrm{~h}$ all cells showed fluorescence at $\lambda_{\mathrm{em}}=$ $530 / 30 \mathrm{~nm}$, indicating uptake of mTFP. Before all RAW264.7 cells had internalized the mTFP (and thus probably BlE), $24 \mathrm{~h}$ had passed. This also means that the mTFP cargo was still showing fluorescent signal, suggesting that the cargo is protected from rapid degradation. Finally, the uptake of BlE-mTFP resulted in morphological changes of the RAW264.7 
cells. We observed this by the changes in forward and side scatter from the flow cytometry, suggesting differentiation of the macrophages.

The confocal microscopy analysis (Figure 5.3) showed that the RAW264.7 internalize the mTFP that was loaded in the BlE, suggesting that the encapsulins were also taken up. They remain in small clusters, indicating containment in lysosomes, endosomes or similar small vesicles, and do not enter the nucleus of the cell. LYSO-RED, which stains acidic organelles like lysosomes, was not selective (Figure 5.3 and 5.4). Therefore, colocalization was impossible to indicate. But since the green signal is not homogeneously spread but appears in "dots", the mTFP cargo remains contained in either the encapsulins or a different vesicle such as lysosomes.

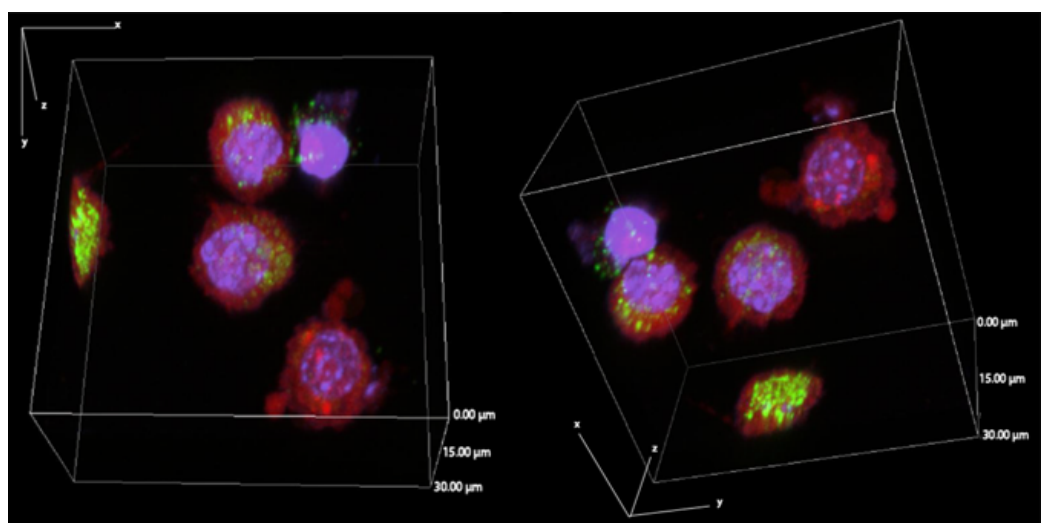

Figure 5.3: The uptake of BlE-mTFP in RAW264.7 cells as determined by confocal microscopy. By creating Z-stacks of the same X and Y coordinates, we made a 3D rendition of the cells. Blue indicates the DAPI that colours the nucleus. Red is the LYSO-ID Red Detection Reagent, which stains lysosomes. Green indicates the mTFP cargo that was contained in the BlE. The cells were incubated with $30 \mathrm{nM}$ BlE-mTFP for $3 \mathrm{~h}$ and subsequently fixed. 

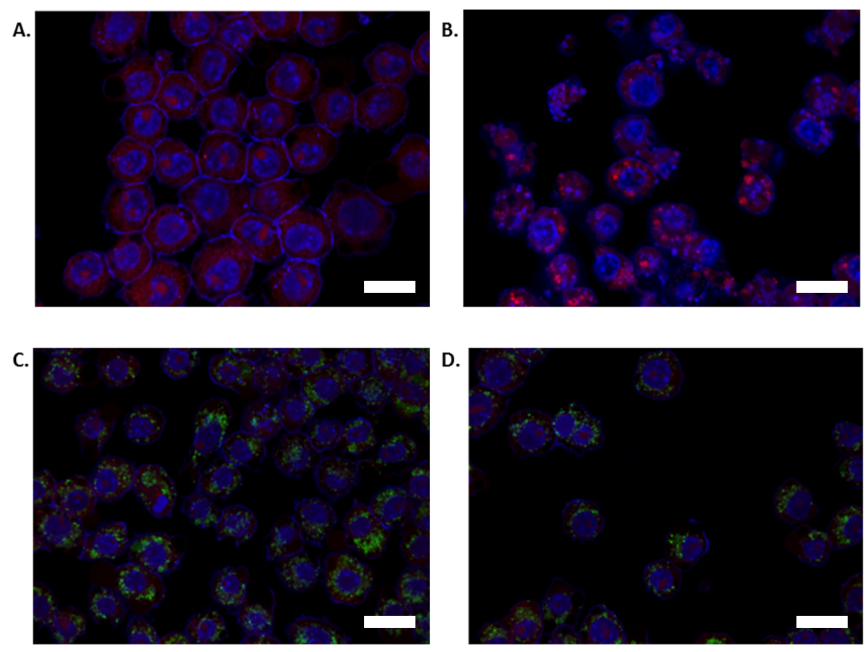

Figure 5.4: RAW264.7 cells stained with LYSO-ID (except for C) A) Untreated B) Chloroquine and C and D) BlE-mTFP encapsulin. Here, $100 \mathrm{nM}$ BlE-mTFP was added and incubated for $24 \mathrm{~h}$. Scalebars represent $20 \mu \mathrm{m}$.

We expanded the study with confocal microscopy in J774 cells, which is a different mouse macrophage cell line. Here we found, after the cells were treated with BlE-mTFP, a similar outcome to RAW 264.7 cells. Again, the LYSO-ID red staining was not selective and there are "dots" of green indicating the mTFP cargo, which co-locate with the encapsulin shell if mTFP is still encapsulated (Figure 5.5). Given the similarity of the results compared with the RAW264.7 macrophages (Figure 5.4), it is likely that other macrophages do also internalize BlE-mTFP. 

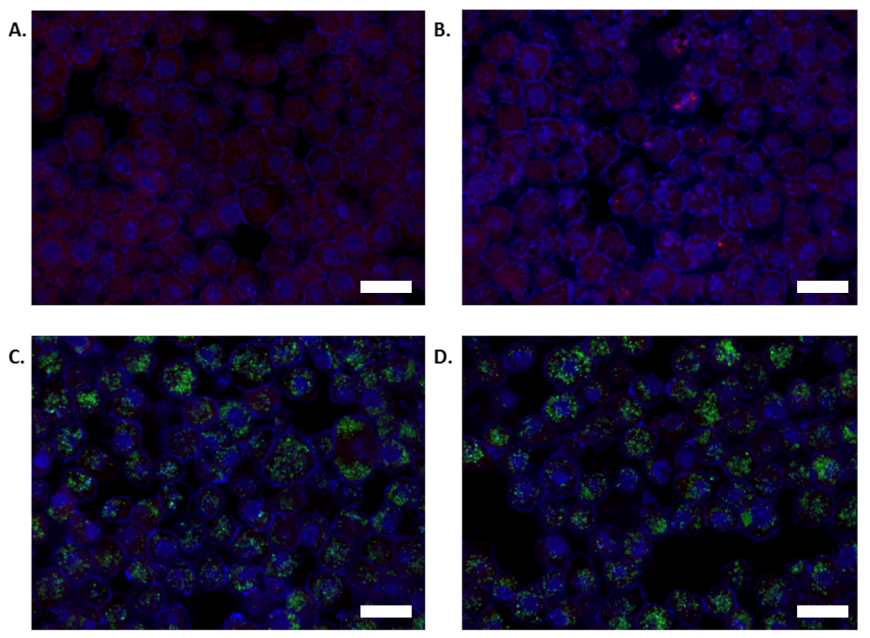

Figure 5.5: J774 cells stained with LYSO-ID (except for C) A) Untreated B) Chloroquine and C and D) BlE-mTFP encapsulin. Here, $100 \mathrm{nM}$ BlEmTFP was added and this was incubated for $24 \mathrm{~h}$ after which the cells were fixed. Scalebars represent $20 \mu \mathrm{m}$.

In both RAW264.7 and J774 macrophages, the mTFP cargo is present in some cells after $3 \mathrm{~h}$ and, given that it is localized in "dots" in the cell, it is likely that the mTFP cargo is present in the lysosomes or endosomes. If the cargo is released from these vesicles, the whole cytoplasm would likely be a homogeneous green. Despite endosomes and lysosomes breaking down proteins and other foreign materials, the uptake in all the cells after $24 \mathrm{~h}$ indicates that the protein cargo (mTFP) is not broken down (Figure 5.2). As mTFP is a protein, it would likely degrade in the lysosomes. Its presence after $24 \mathrm{~h}$ suggests that the BlE shell serves as a protective cage, protecting the mTFP-cargo. Given that they are present in macrophages, which are known to degrade protein entities, ${ }^{12,13}$ the encapsulin shell has a highly protective function. 
(a)

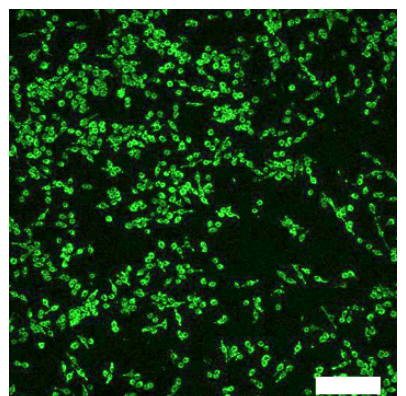

$\mathrm{Oh}$ (b)

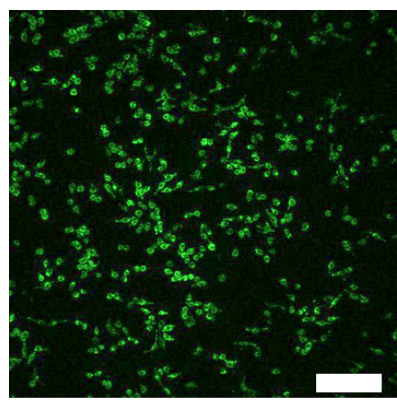

$2 \mathrm{~h}$ (c)

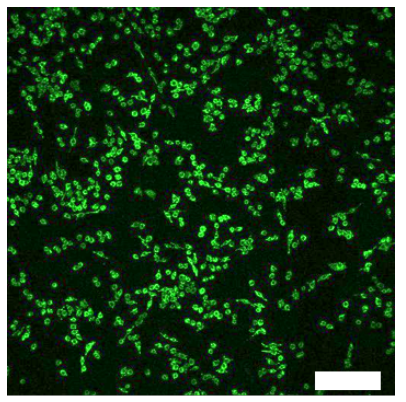

$4 \mathrm{~h}$ (d)

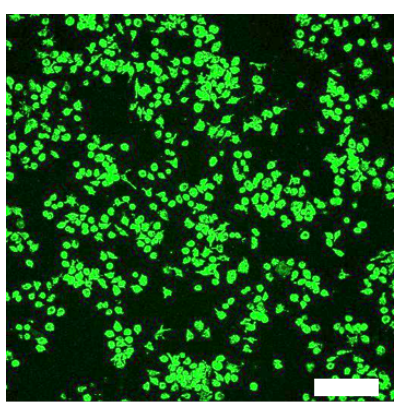

$24 \mathrm{~h}$ (e)

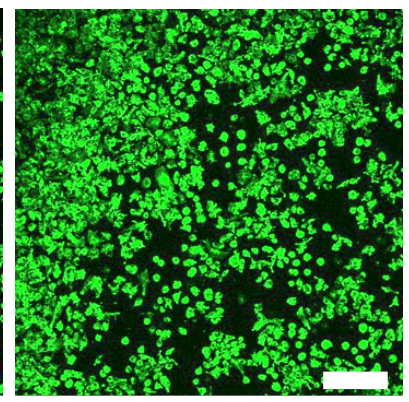

$48 \mathrm{~h}$

Figure 5.6: Confocal microscopy images of RAW264.7 cells incubated for 4 hours with Bl-mTFP and subsequently fixed after $0 \mathrm{~h} \mathrm{(A),} 2 \mathrm{~h}$ (B), $4 \mathrm{~h}$ (C), $24 \mathrm{~h}(\mathrm{D})$ and $48 \mathrm{~h}(\mathrm{E})$. The green signal corresponds to the mTFP. The scalebars represent $200 \mu \mathrm{m}$.

To further study what happens to encapsulins and the mTFP cargo after they are internalized by RAW264.7 cells, we incubated the cells with 50 $\mathrm{nM}$ BlE-mTFP for $4 \mathrm{~h}$, after which we removed the sample and replaced it with medium. Then, after various timepoints, we fixed the cells and analysed them with confocal microscopy (Figure 5.6). We found that directly after $4 \mathrm{~h}$ incubation the RAW264.7 cells had taken up the mTFP cargo and therefore likely the BlE (Figure 5.6A). The intensity of this 
signal remains similar after 2 and $4 \mathrm{~h}$ post-incubation (Figure 5.6B and C). Interestingly, the intensity increases in the samples 24 and 48 h postincubation (Figure 5.6D and E). Given that the source of the green signal (BlE-mTFP) was removed, the increased signal cannot be explained by renewed uptake of BlE-mTFP. We suggest that the mTFP resides in the encapsulins during the first three timepoints (up to at least $4 \mathrm{~h}$ ). Given that each encapsulin nanoparticle contains $\sim 12$ mTFP monomers,${ }^{14}$ it is possible that self-quenching occurs. ${ }^{15}$ However, it has been suggested that the "barrel" shape of fluorescent proteins has a protective function preventing the quenching, ${ }^{16}$ which suggests that the mTFP structure might be altered. This quenching is lost after $24 \mathrm{~h}$ post-incubation, when the $\mathrm{BlE}$ shell is broken down and the mTFP cargo is released. It is probable that the BlE breakdown occurs in the endosomes and that the free mTFP is instead released into the cytoplasm avoiding breakdown. This hypothesis is supported by the fact that after $48 \mathrm{~h}$ post-incubation, the signal is as intense as $24 \mathrm{~h}$, and thus the mTFP is not degraded.

Given that macrophages are known to internalize larger particles, we also studied the difference in uptake between mTFP and BlE-mTFP in RAW264.7 cells (Figure 5.7). We found that mTFP is not taken up at either $50 \mathrm{nM}$ or $250 \mathrm{nM}$ concentration for the first $2 \mathrm{~h}$ and that there is a slight uptake after $24 \mathrm{~h}$. This uptake is negligible compared with mTFP encapsulated in BlE, which shows uptake after $2 \mathrm{~h}$ at $50 \mathrm{nM}$, and high uptake after 4 and $24 \mathrm{~h}$. The number of cells at $24 \mathrm{~h}$ is reduced compared with $4 \mathrm{~h}$, likely because of shortage of culture medium, since the encapsulins were added in a 1:1 ratio to the medium. The reduction of cell count is unlikely due to BlE being toxic, as there is also a low cell count with the mTFP only samples. While the mTFP might be toxic, ${ }^{17}$ the mTFP is not internalized by the cells and mTFP has no extracellular toxicity. The experiment demonstrates that the encapsulation of mTFP enhances its uptake in RAW264.7 macrophages. 


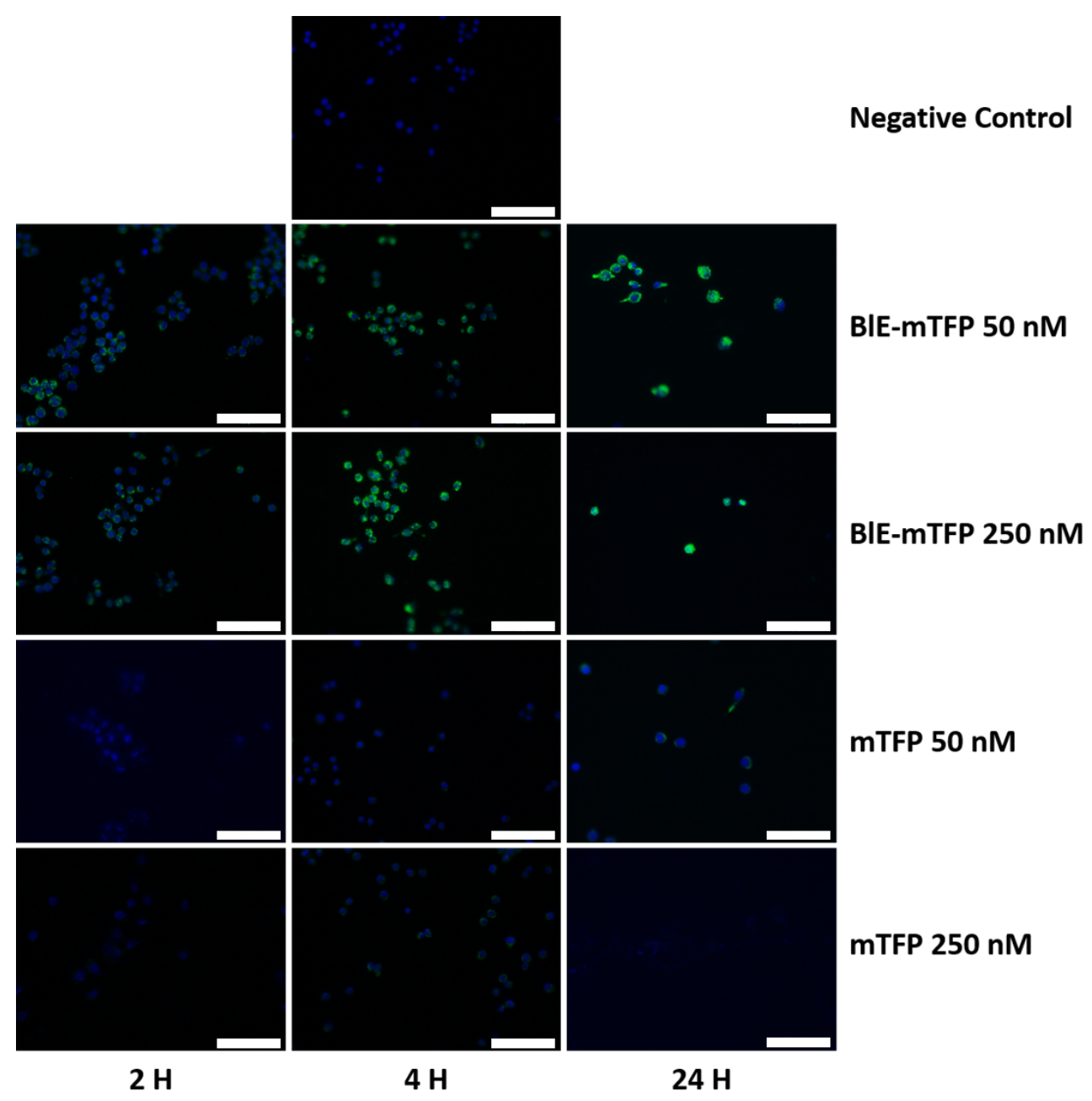

Figure 5.7: Fluorescence of RAW264.7 cells after treatment with BlEmTFP and mTFP with different concentrations and incubation times. The blue signal corresponds to the DAPI stain (nucleus) while the green signal corresponds to mTFP. Scalebars represent $100 \mu \mathrm{m}$. 


\subsubsection{Uptake Route of Brevibacterium linens En- capsulin}

We learned that encapsulins most likely end up in lysosomes, but the exact route of uptake remains unknown. To study this uptake route, we blocked various uptake pathways to determine which one is used by BlE. First, we tested the efficiency of the blockers by studying the uptake of dextran and polystyrene, which use specific pathways to enter cells. Table 5.1 provides an overview of the blockers, the pathways they block and the pathways used by the control material.

Table 5.1: Overview of pathway blockers and control materials.

\begin{tabular}{ll} 
Blocker & Blocking Pathway \\
Amiloride & Macropinocytosis \\
Cytochalasin D & Macropinocytosis and phagocytosis \\
Concanavalin A & All pathways \\
Hyper sucrose & All pathways \\
\hline Control & Uptake Pathway \\
Dextran & Macropinocytosis \\
Polystyrene & Phagocytosis
\end{tabular}

While dextran is taken up via macropinocytosis and polystyrene by phagocytosis, the blockers did not show a clear specificity; either they are not specific (in RAW264.7 cells), or other uptake mechanisms are enhanced by a feedback loop. Dextran is also taken up when the micropinocytosis uptake pathway is blocked by amiloride, which should inhibit dextran uptake. Polystyrene uptake is reduced by every used blocker, even amiloride, which should not affect phagocytosis (Figure 5.8). These data affect the analysis with encapsulins, given that the blockers are not completely blocking the selective pathways, or alternative uptake routes are used when the main route is blocked. 

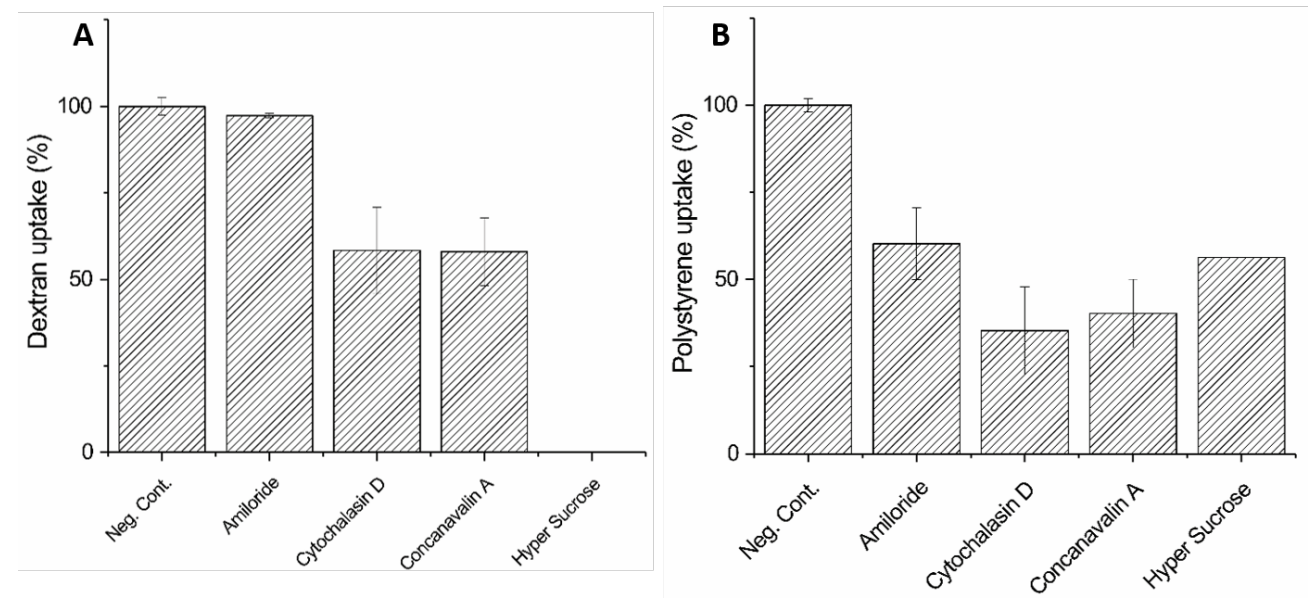

Figure 5.8: The uptake of dextran (A) and polystyrene (B) in RAW264.7 cells, when cell uptake pathways were blocked by various blockers. Error bars represent the standard deviation $(\mathrm{N}=3)$.

Encapsulin uptake appears to be affected more by the cell type than the blocking of uptake pathways. In the RAW264.7 cells, the blocking of various uptake pathways does not affect encapsulin uptake, suggesting that either the blocking did not work or that the encapsulins are internalized by a different mechanism when one is blocked (Figure 5.9A). In J774 macrophages, the cell uptake is reduced by every blocker, suggesting that encapsulins are not taken up by just one pathway, but that multiple pathways are involved (Figure 5.9B). This was also observed by de Ruiter et al. with $\mathrm{CCMV},{ }^{9}$ a nanoparticle with similar dimensions as encapsulin (Chapter 4). 

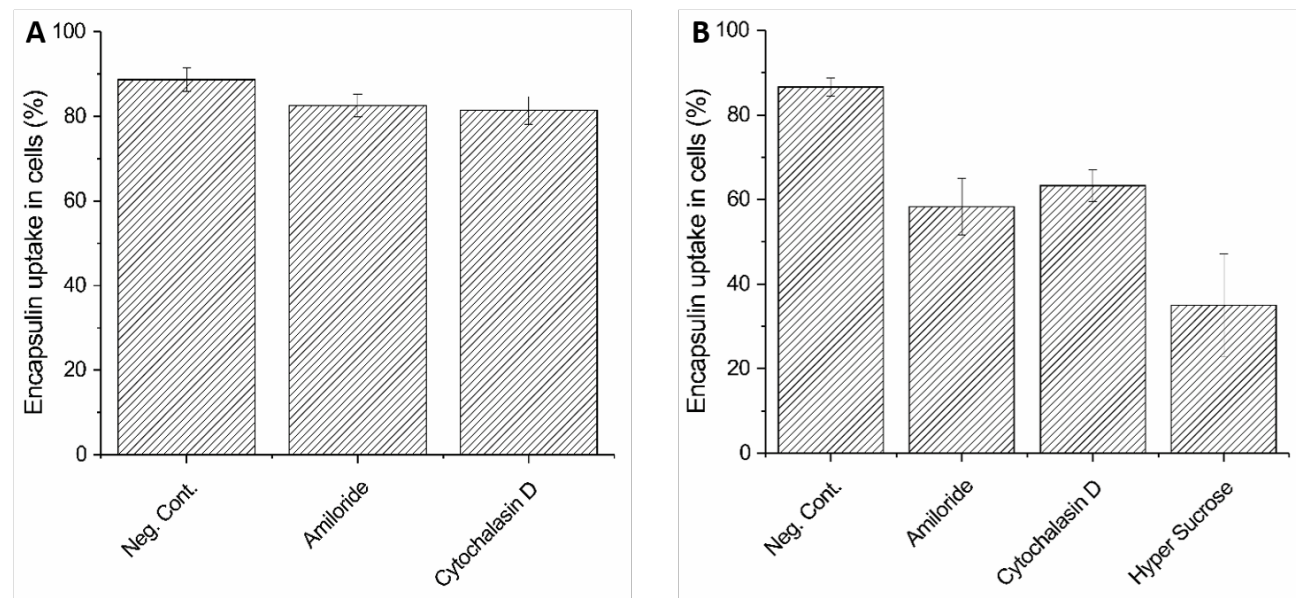

Figure 5.9: The uptake of BlE-mTFP in RAW264.7 (A) and J774 (B) cells, when cell uptake pathways were blocked by various blockers. Error bars represent the standard deviation $(\mathrm{N}=3)$.

While the number of J774 cells that internalized BlE-mTFP was lower when uptake pathways were blocked, we also found a difference in intensity of the mTFP cargo in the cells. With both hyper sucrose and cytochalasin D the intensity of the mTFP signal is lower compared with both the amiloride and no blocker. The intensity is increased when more mTFP and thus more particles are taken up by a cell. So, while not as many J774 cells internalized encapsulins when blocked by amiloride, the intensity of the fluorescent signal in cells that were not treated with a blocker is similar. This suggests that the cells that did internalize encapsulin, did so in a similar amount. The other two blockers (hyper sucrose and cytochalasin D) resulted in fewer encapsulins being taken up per cell (Figure 5.10A). 

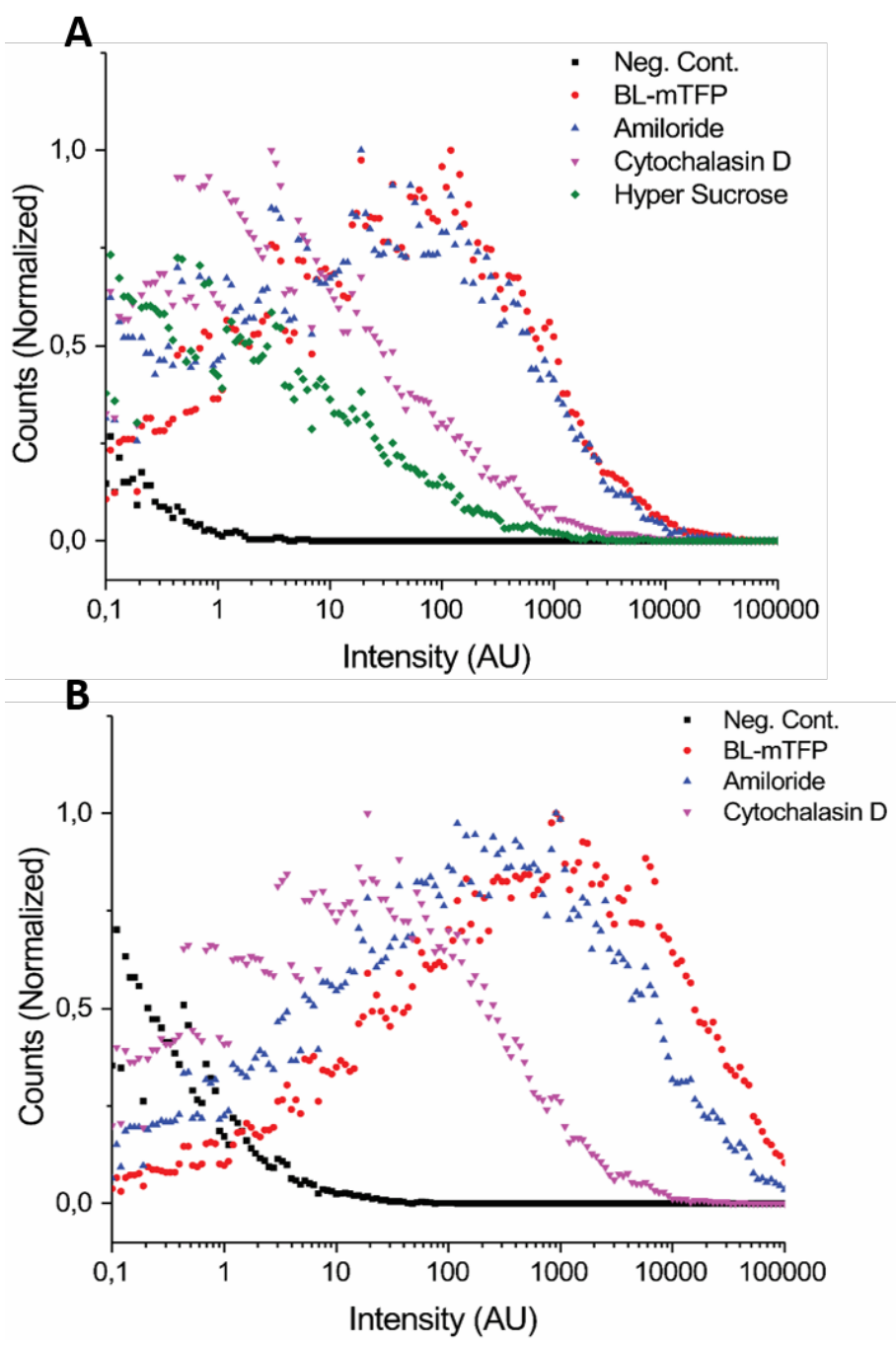

Figure 5.10: Intensity of BlE-mTFP when J774 (A) and RAW264.7 (B) cells are blocked with various blockers. We used 10,000 hits per sample for the flow cytometry analyses.

In RAW264.7 macrophages, the differences in intensity are clearer. While the blockers did not reduce the amount of cells that took up the encapsulin, there is a difference in the amount of encapsulins that was taken up per cell. Again, the blocking by amiloride did not reduce the amount 
of encapsulins taken up per cell, as the intensity is similar to the control without a blocker. When treated with hyper sucrose or cytochalasin D, the RAW264.7 cells take up fewer encapsulins per cell (Figure 5.10B).

These data suggest that the encapsulins are predominantly taken up by micropinocytosis and phagocytosis. However, this is dependent on the cell type to which the encapsulins are administrated and blocking one pathway does not completely inhibit the uptake of encapsulins, which suggests that multiple routes are used or that one pathway is enhanced when another is blocked.

Therefore, these data are not conclusive enough to determine which uptake pathway is the main route by which encapsulins are internalized by macrophages. However, given that if blocking one pathway stimulates encapsulin uptake by a different pathway, multiple pathways are involved. Additionally, there is a large difference between cell types on the final encapsulin uptake.

\subsubsection{Immunogenicity of Brevibacterium linens En- capsulins in RAW264.7 Cells}

Given that encapsulins are derived from bacteria, it is probable that these encapsulin will induce an immunological response. To study this response, we first investigated the differentiation of RAW264.7 macrophages by studying the morphology of the cells in combination with analysis of clusters of differentiation (CD). When RAW264.7 macrophages differentiate into either M1 or M2 macrophages, their morphology changes, which we determined with both microscopy and flow cytometry (Figure 5.11). 


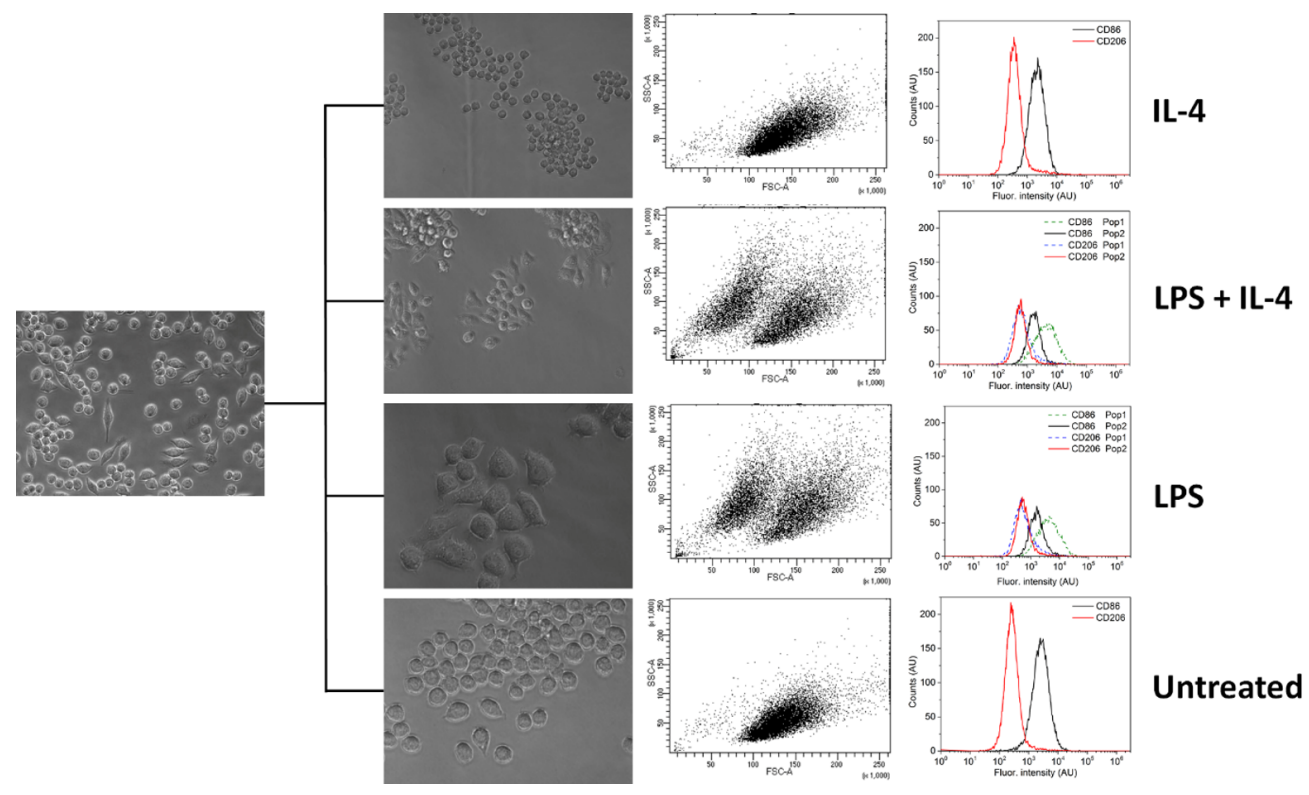

Figure 5.11: RAW264.7 macrophages were incubated with LPS (M1 differentiation), IL-4 (M2 differentiation), a combination of both, or neither. We used 10,000 hits per sample for the flow cytometry analyses.

Figure 5.11 shows the different morphologies of the M1 macrophages (induced by lipopolysaccharide (LPS)) ${ }^{18}$ and M2 macrophages (induced by interleukin 4 (IL-4) ${ }^{19}$ when analysed using microscopy. The M1 differentiated macrophages also create a new subpopulation in the flow cytometry experiment. This subpopulation is seen in the forward scatter set against the side scatter, further verifying the morphological change.

Additionally, we used flow cytometry to study the CD86 and CD206 expression. These correspond to the M1 (CD86) and M2 differentiation (CD206). ${ }^{20}$ While we found increased values of CD86 when the samples were treated with LPS, we found no changes in CD206 expression induced by IL-4. The CD analyses were not sufficient for further analysis with BlE-DyP and BlE-mTFP, prompting us to use a different method to study immunogenicity. 


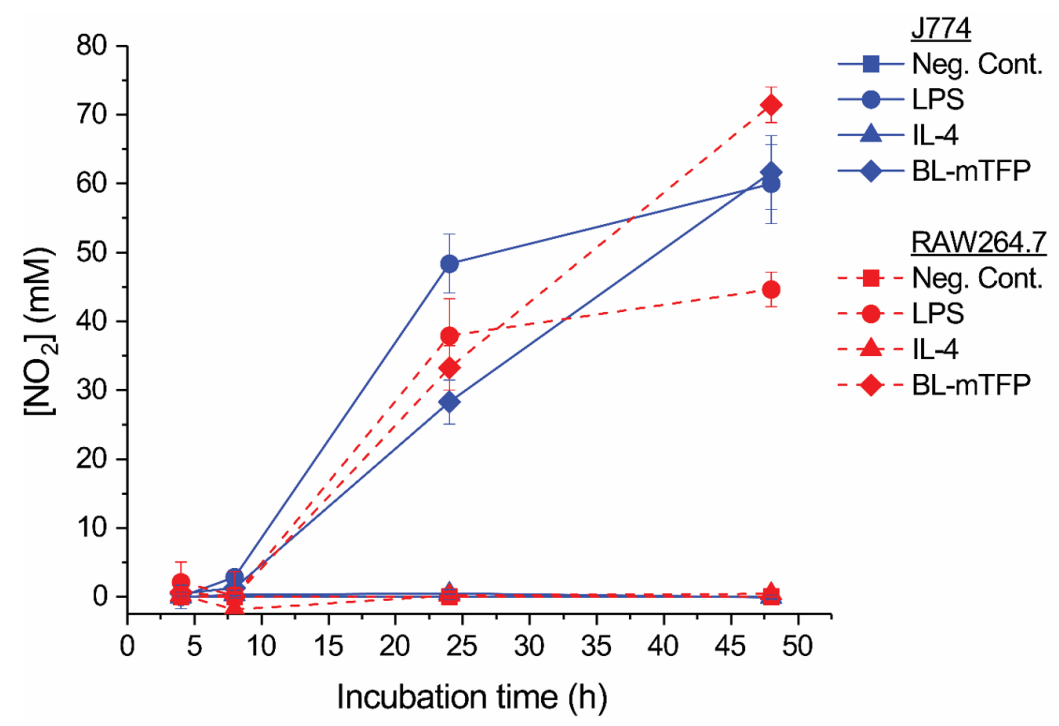

Figure 5.12: NO levels in macrophages treated with different stimulants. We used two types of mouse macrophages, J774 and RAW264.7. Error bars represent the standard deviation $(\mathrm{N}=3)$.

This method uses the principle that M1 macrophages produce nitric oxide (NO), while M2 macrophages do not. ${ }^{21}$ Therefore, determining the NO concentration using the Griess reagent can be used to study the RAW264.7 differentiation. The LPS stimulated J774 and RAW264.7 macrophages, both resulting in increased NO levels, verifying the assay and the M1 differentiation of the macrophages after LPS treatment. Stimulating the cells with IL-4, which induces M2 differentiation, does not result in increased NO levels, as M2 macrophages do not produce NO. Stimulation with encapsulins results in increased NO levels, suggesting that the encapsulins induced M1 differentiation of macrophages (Figure 5.12). These immunogenicity studies are very preliminary and should be extended in later research, especially if encapsulins are to be used for medical purposes, but they do suggest that encapsulins induce an immunogenic response. 


\subsubsection{Discussion}

In this chapter we studied the uptake, retention and immunogenicity of BlE in cells. This approach was quickly limited, because BlE are not taken up in every cell type. We found no significant uptake in HeLa and $\mathrm{C} 2 \mathrm{C} 12$ cells, which corresponds to research done by Moon et al., ${ }^{22}$ while we did find uptake in two types of macrophages (J774 and RAW264.7). Macrophages are more likely to take up encapsulins as macrophages take up particles as part of their biological function. ${ }^{13}$ The specificity of cells that do or do not take up encapsulins requires further study, but this specificity can be utilized. If encapsulins are indeed not taken up in most cell types, it could mean that non-specific targeting is reduced when enzymes or other particles are encapsulated in encapsulins and thus that the encapsulins can be used as drug carriers. It does then, however, require a targeting system, such as the one introduced by Moon et al. ${ }^{10,22}$ This increased specificity can be of benefit to many therapies as it could reduce side effects and toxicity, while requiring a lower overall dose.

Additionally it is worthwhile to study the exact reasons why encapsulins are not or poorly taken up by most cells, while similarly shaped and sized protein nanoparticles, such as VLPs, are taken up. ${ }^{9}$ It is probable that the surface of encapsulins is the primary reason for this lack of internalization. Analysing this could lead to specific modifications on other drug carriers, like VLPs or bacteriophages, that could limit unwanted cellular uptake, increasing their possibilities to be used in medicine.

With the clear difference in uptake between mTFP and BlE-mTFP, we demonstrate that encapsulins as a carrier can help increase the internalization of cargo in a cell. Often, protein cargo gets degraded in the lysosomes, but our data suggest that with encapsulins, this issue is circumvented. We found that mTFP appears to be released from the encapsulins and resides in the cytoplasm of the RAW264.7 cells, where it 
remains stable for at least $24 \mathrm{~h}$. This suggests that the encapsulin shell protected the cargo in the lysosomes and releases the intact cargo in the cell. It is plausible that the lysosomes degrade the encapsulins which results in cargo release. This would make encapsulins a great candidate for the delivery of therapeutic proteins and other molecules. However, this requires additional study to conclusively determine the fate of encapsulins after internalization. This should also be studied with different cell combinations with encapsulins that have exterior targeting modifications.

The selective uptake by macrophages in turn can be utilized for vaccination purposes. If encapsulins are internalized only by macrophages and they increase the internalization of the target cargo (an epitope), then this could lead to an increased immune response. Encapsulating epitopes in or adding them to the exterior of encapsulins could provide the basis for new vaccine formulations, as was demonstrated by Lagoutte et al. ${ }^{23}$ While cancer cells can have increased uptake of particles because of the enhanced permeability and retention effect, ${ }^{24}$ we found no increased uptake in HeLa cells. Macrophages also internalize particles more actively than other cells and we did find increased uptake in macrophages, but only after $24 \mathrm{~h}$; the uptake is gradual. The exact mechanism differs per macrophage cell type, as demonstrated in the differences between J774 and RAW264.7 macrophages. Any further studies should take this into account.

The uptake in macrophages is interesting because of the related immune response, which can be both an advantage and a disadvantage. It is advantageous if the encapsulins are used for vaccine purposes, in which the increased uptake can trigger an increased immune response compared with non-encapsulated antigens. If the cargo is a therapeutic enzyme (such as L-asparaginase, described in Chapter 4), an immune response is disadvantageous. This can potentially be countered by the modification 
of the exterior of encapsulins with polyethylene glycol or human serum albumin, but this requires additional study.

\subsection{Conclusions}

The uptake of BlE is low in all the tested cells that were not macrophages. In the macrophages the BlE-mTFP showed higher fluorescence intensity caused by the mTFP cargo that was internalized compared with nonencapsulated mTFP. In addition to the increased uptake, the encapsulins are also able to protect the cargo from probable lysosomal degradation and result in the cargo ending up in the cytoplasm. This principle makes encapsulins interesting for future research in their function as a drug carrier. This uptake in macrophages does result in an immunological response, showing macrophage differentiation to M1, which means that there is potential to use encapsulins as a vaccine delivery carrier.

\subsection{Acknowledgements}

I thank V. M. Dombrowe, J. Smelt, E. Pijnappel, M. Szalas, A. Gabriël, C. Rohof, J. de Leeuw and R. van der Hee for their contributions to this chapter. 


\subsection{Experimental Section}

\subsubsection{Materials}

All chemicals were obtained from Sigma-Aldrich unless stated otherwise. Buffers were prepared using double deionized water from a Millipore system.

\subsubsection{Production of Encapsulins}

The BlE were produced in the same fashion as described in Chapter 3. We used a plasmid provided by dr. Rurup that contained the operon coding for mTFP (with the spacer and affinity sequence on its C-terminus) and BlE. This complex could be excited at $\lambda_{\mathrm{ex}}=460 \mathrm{~nm}$ and visualized using an emission at $\lambda_{\mathrm{em}}=530 / 30 \mathrm{~nm}$.

\subsubsection{Cell Culture}

RAW264.7 cells (ATCC) were cultured in Dulbecco's Modified Eagle's Medium (DMEM) which contained $4 \mathrm{mM}$ l-glutamine, $4,500 \mathrm{mg} / \mathrm{L}$ glucose, $1 \mathrm{mM}$ sodium pyruvate and 1,500 mg/L $\mathrm{NaHCO}_{3}$. This was supplemented with $20 \%$ (v/v) fetal bovine serum (FBS), $10 \mu \mathrm{g} / \mathrm{mL}$ streptomycin and $0.2 \mathrm{mg} / \mathrm{L}$ penicillin. When at $80 \%$ confluence, the cells were split using trypsin to detach them from the T75 culture flasks. After $2 \mathrm{~min}, 4 \mathrm{~mL}$ medium was added to deactivate the trypsin, after which the cells were collected, centrifuged (Eppendorf centrifuge 5702; 1,500 rpm, $5 \mathrm{~min}$ ), the supernatant was removed and cells were resuspended in DMEM. Depending on the dilution, $0.5-1 \mathrm{~mL}$ was added to a new flask, filled to $10-12$ $\mathrm{mL}$ DMEM and incubated at $37^{\circ} \mathrm{C}$ and $5 \% \mathrm{CO}_{2}$.

J774 cells were cultured similarly to RAW264.7. To detach the cells, instead of trypsin, versene (Gibco) was added to the cells and this was incubated for 5 - 10 min after which the cells were centrifuged. 
HeLa cells and C2C12 were cultured similarly to RAW264.7 cells. The culture medium (DMEM) was instead supplemented with $2 \mathrm{mM}$ L-glutamine, $1 \%(\mathrm{v} / \mathrm{v})$ pen-strep and 10\% FBS. During the cell splitting, the centrifugation step was performed at 1,400 rpm for $4 \mathrm{~min}$.

\subsubsection{Encapsulin Uptake Study}

For the analysis of the uptake of BlE-mTFP in various cells, first the cells were cultured in a flat-bottom 96-wells plate at 5,000 cells per well. This amount of cells was determined after optimization experiments. These cells were incubated overnight at $37^{\circ} \mathrm{C}$ and $5 \% \mathrm{CO}_{2}$, after which different concentrations of BlE-mTFP in PBS were added, always in triplo. For the initial uptake studies to determine optimal incubation times and concentration, a range from 0 - $500 \mathrm{nM}$ BlE-mTFP was added and incubated for 0.5 - $24 \mathrm{~h}$. Next, the exposure medium was removed, washed with PBS, fixed, stained with DAPI and WGA and analysed with confocal microscopy.

To study what happens to BlE-mTFP after internalization, the cells were incubated as before, but this time treated for only $4 \mathrm{~h}$ with $50 \mathrm{nM}$ BlEmTFP. After $4 \mathrm{~h}$, the medium with BlE-mTFP was removed and replaced with new medium without BlE-mTFP. After various timepoints (0 - 48 h) the cells were fixed and analysed with confocal microscopy.

For the comparison between the uptake of mTFP and BlE-mTFP, the cells were cultured as before, with BlE-mTFP or non-encapsulated mTFP (that was purified during the FPLC step of the encapsulin purification) being added at 50 or $250 \mathrm{nM}$ concentrations and incubated for 4 or $24 \mathrm{~h}$. Next, the cells were washed, fixed and analysed with confocal microscopy. The concentration of mTFP and BlE-mTFP was equalized based on the $\lambda=458 \mathrm{~nm}$ peak determined using UV-vis. Analysis of just BlE showed 
no signal at $\lambda=458 \mathrm{~nm}$, suggesting that this is a suitable method for the determination of mTFP concentration. ${ }^{25}$

\subsubsection{Uptake Mechanism Study}

To study the uptake mechanism that is used to internalize BlE-mTFP, RAW264.7 and J774 cells were seeded in 24-wells plates at 100,000 cells per well. These were incubated overnight at $37^{\circ} \mathrm{C}$ and $5 \% \mathrm{CO}_{2}$. Next, 40 $\mu \mathrm{M}$ cytochalasin $\mathrm{D}, 7.5 \mathrm{mM}$ amiloride, $250 \mu \mathrm{g} / \mathrm{mL}$ concanavalin A or 0.45 $\mathrm{mM}$ sucrose was added to the wells in a maximal volumetric ratio of 1:10 (blocker to medium). This was incubated for $30 \mathrm{~min}$ at $37^{\circ} \mathrm{C}$ and $5 \%$ $\mathrm{CO}_{2}$. Subsequently, $5 \mu \mathrm{M}$ dextran (Thermo Fisher), $1 \mu \mathrm{M}$ polystyrene or $100 \mathrm{nM}$ BlE-mTFP was added and this was incubated for $2.5 \mathrm{~h}$ at the aforementioned settings. Following the incubation, the medium was removed and the cells were detached with versene (Gibco). The samples were centrifuged for $3 \mathrm{~min}$ at $300 \mathrm{x} \mathrm{g}$ and the pellet was resuspended in live cell imaging solution (Thermo Fisher) and stained for life/dead analysis. The cells were analysed with flow cytometry using $\lambda_{\text {ex - em }} 488$ - 585/42 $\mathrm{nm}$ (Propidium Iodide), 488 - 530/30 nm (BlE-mTFP and polystyrene), 375 - 450/40 nm (Calceïn violet AM) and 633 - 660/20 nm (dextran) filters.

\subsubsection{Flow Cytometry}

Prior to flow cytometry analysis, the cells were treated with the required fluorescent labels by adding them to the medium and incubating for various times. Subsequently, the cells were removed from the wells plate and transferred, in medium, to $15 \mathrm{~mL}$ tubes. Then, the cells were centrifuged at $125 \mathrm{x} \mathrm{g}$ for $5 \mathrm{~min}$. The supernatant was removed and the cell pellet was resuspended in PBS. Just prior to analysis with the flow cytometer, propidium iodide (PI) was added to the sample and briefly incubated. PI served as a stain for dead cells, which were excluded during the analysis. 
The cells were analysed with a FACS ARIA II (BD Biosciences) flow cytometer until 10,000 counts were obtained. Using the forward scatter (FSC) and side scatter (SSC), gates were set creating a subpopulation of only single cells. Subsequently, a gate was set around the cells that showed no fluorescence (PI-negative) at $\lambda_{\mathrm{ex}}=488 \mathrm{~nm}$ and $\lambda_{\mathrm{em}}=585 / 42$ $\mathrm{nm}$. This way, only single, viable cells were selected for further analysis.

Depending on the assay, different analyses were performed. For CD86FITC and CD206-PE, the fluorescence was measured at $\lambda_{\mathrm{ex}}=488 \mathrm{~nm}$ and $\lambda_{\mathrm{em}}=530 / 30$ and $\lambda_{\mathrm{ex}}=488 \mathrm{~nm}$ and $\lambda_{\mathrm{em}}=585 / 42$, respectively, without the prior PI selection.

\subsubsection{Fluorescence Microscopy}

For the non-confocal fluorescence microscopy, cells were plated at a density of 2,500 cells per well on a 96-wells plate (Greiner) and incubated overnight at $37^{\circ} \mathrm{C}$ and $5 \% \mathrm{CO}_{2}$. The next day, $\mathrm{BlE}$ or other treatments were added and incubated for varying time points. After incubation, the cells were washed with PBS for 5 min, after which they were fixed with 200 $\mu \mathrm{L} 4 \%$ formaldehyde for $10 \mathrm{~min}$. Next, the cells were washed three times with PBS for $5 \mathrm{~min}$. After fixing the cells, they were stained with $100 \mu \mathrm{L}$ 4',6-diamidino-2-phenylindole (DAPI (Thermo Fisher), diluted 1:10,000) for $10 \mathrm{~min}$ to stain the nucleus. After two washing steps with PBS, 50 $\mu \mathrm{L}$ Wheat germ agglutinin (WGA (Biotium)) was added and incubated for 20 min to stain the cell membrane, followed by two more wash steps. The cells were analysed with a Olympus IX71S1F-3 microscope equipped with an X-cite Series 120 pc Q laser and an Olympus TH4-200 lamp. Fluorescence excitation and emission were done at $\lambda_{\mathrm{ex}}=360-370$ and $460-490 \mathrm{~nm}$ and $\lambda_{\mathrm{em}}=460$ and $525 \mathrm{~nm}$. 


\subsubsection{Confocal Microscopy}

For the confocal microscopy, cells were cultured in flat bottom 96-wells plates. After treatment and fixing the cells, they were analysed using a Nikon Confocal A1 microscope with NIS-Elements software. The laser settings that were used were $\lambda_{\mathrm{ex}}-\lambda_{\mathrm{em}}$ of 405 and $425-475 \mathrm{~nm}$ for DAPI and WGA, 488 and $500-550 \mathrm{~nm}$ for mTFP and 561 and $663-738 \mathrm{~nm}$ for LYSO-ID RED, respectively. Images were processed with ImageJ.

\subsubsection{NO Detection with Griess Reagent}

The Griess reagent is used to detect $\mathrm{NO}_{2}{ }^{-}$, the oxidized product of nitrogen oxide (NO). A calibration curve was made by creating nitrite free water by degassing Milli-Q with helium and then dissolving $\mathrm{NaNO}_{2}$ in it. Next, 30,000 RAW264.7 cells were plated per well on a 96-wells plate. The cells were treated with various materials; BlE-mTFP, LPS, IL-4 and PBS as a negative control. After 4, 8, 24 and $48 \mathrm{~h}$ incubation at $37^{\circ} \mathrm{C}, 5 \%$ $\mathrm{CO}_{2}$, the liquid was recovered and mixed 1:1 with Griess reagent modified. After incubating for $15 \mathrm{~min}$, the absorbance at was measured $\lambda=$ $540 \mathrm{~nm}$ using a Tecan Infinite 200 Pro, in triplo. 


\subsection{References}

1. Behzadi, S.; Serpooshan, V.; Tao, W.; Hamaly, M. A.; Alkawareek, M. Y.; Dreaden, E. C.; Brown, D.; Alkilany, A. M.; Farokhzad, O. C.; Mahmoudi, M. Cellular Uptake of Nanoparticles: Journey Inside the Cell. Chem. Soc. Rev. 2017, 46, 4218 - 4244

2. Alberts, B.; Johnson, A.; Lewis, J.; Morgan, D.; Raff, M.; Roberts, K.; Walter, P. Molecular Biology of the Cell. $5^{\text {th }}$ Edition. 2012, Garland Science

3. Alberts, B.; Bray, D.; Hopkin, K.; Johnson, A.; Lewis, J.; Raff, M.; Roberts, K.; Walter, P. Essential Cell Biology. $4^{\text {th }}$ Edition. 2014, Garland Science

4. Sutter, M.; Boehringer, D.; Gutmann, S.; Günther, S.; Prangishvili, D.; Loessner, M. J.; Stetter, K. O.; Weber-Ban, E.; Ban, N. Structural Basis of Enzyme Encapsulation into a Bacterial Nanocompartment. Nat. Struct. and Mol. Biol. 2008, 15, 939 - 947

5. Putri, R. M.; Allende-Ballestero, C.; Luque, D.; Klem, R.; Rousou, K-A.; Liu, A.; Traulsen, C. H.-H.; Rurup, W. F.; Koay, M. S. T.; Castón, J. R.; Cornelissen, J. J. L. M. Structural Characterization of Native and Modified Encapsulins as Nanoplatforms for in Vitro Catalysis and Cellular Uptake. ACS Nano. 2017, 11, 12796 - 12804

6. Gustafson, H. H.; Holt-Casper, D.; Grainger, D. W.; Ghandehari, H. Nanoparticle Uptake: The Phagocyte Problem. Nano Today. 2015, $10,487-510$

7. Parham, P. The Immune System. $3^{\text {rd }}$ Edition. 2009, Garland Science

8. García-Piñeres, A.; Hildesheim, A.; Dodd, L.; Kemp, T. J.; Harro, C.; Williams, M.; Lowy, D. R.; Schiller, J. T.; Pinto, L. A. Cytokine and Chemokine Profiles Following Vaccination with Human Papillomavirus Type 16 L1 Virus-Like Particles. Clin. Vaccine Immunol. 2007, 14, 984 $-989$ 
9. de Ruiter, M. V.; van der Hee, R. M.; Driessen, A. J. M.; Keurhorst, E. D.; Hamid, M.; Cornelissen, J. J. L. M. Polymorphic Assembly of Virus-Capsid Proteins Around DNA and the Cellular Uptake of the Resulting Particles. J. Control. Release. 2019, 307, 342 - 354

10. Moon, H.; Lee, J.; Kim, H.; Heo, S.; Min, J.; Kang, S. Genetically Engineering Encapsulin Protein Cage Nanoparticle as a SCC-7 Cell Targeting Optical Nanoprobe. Biomat. Res. 2014, 18:21

11. Rattan, R.; Bhattacharjee, S.; Zong, H.; Swain, C.; Siddiqui, M. A.; Visovatti, S. H.; Kanthi, Y.; Desai, S.; Pinsky, D. J.; Goonewardena, S. N. Nanoparticle-macrophage Interactions: A Balance between Clearance and Cell-specific Targeting. Bioorg. Med. Chem. 2017, 25, 4487 4496

12. Brazil, M. I.; Weiss, S.; Stockinger, B. Excessive Degradation of Intracellular Protein in Macrophages Prevents Presentation in the Context of Major Histocompatibility Complex Class II Molecules. Eur. J. Immunol. 1997, 27, 1506 - 1514

13. Buktenica, S.; Olenick, S. J.; Salgia, R.; Frankfater, A. Degradation and Regurgitation of Extracellular Proteins by Cultured Mouse Peritoneal Macrophages and Baby Hamster Kidny Fibroblasts. Kinetic Evidence that the Transfer of Proteins to Lysosomes is not Irreversible. J. Biol. Chem. 1987, 262, 9469 - 9476

14. Rurup, W. F.; Snijder, J.; Koay, M. S. T.; Heck, A. J. R.; Cornelissen, J. J. L. M. Self-Sorting of Foreign Proteins in a Bacterial Nanocompartment. J. of the Am. Chem. Soc. 2014, 136, 3828 - 3832

15. Kruitwagen, T.; Denoth-Lippuner, A.; Wilkins, B. J.; Neumann, H.; Barral, Y. Axial Contraction and Short-range Compaction of Chromatin Synergistically Promote Mitotic Chromosome Condensation. eLife 2015, e1039

16. Jung, K.; Park, J.; Maeng, P.; Kim, H. Fluorescence Quenching of Green Fluorescent Protein During Denaturation by Guanidine. Bull. Korean. Chem. Soc. 2005, 26, $413-417$ 
17. Ansari, A. M.; Ahmed, A. K.; Matsangos, A. E.; Lay, F.; Born, L. J.; Marti, G.; Harmon, J. W.; Sun; Z. Cellular GFP Toxicity and Immunogenicity: Potential Confounders in in Vivo Cell Tracking Experiments. Stem Cell Rev. 2016, 12, 553 - 559

18. Martinez, F. O.; Gordon, S. The M1 and M2 Paradigm of Macrophage Activation: Time for Reassessment. F1000Prime Rep. 2014, 6:13 19. Genin, M.; Clement, F.; Fattaccioli, A.; Raes, M.; Michiels, C. M1 and M2 Macrophages Derived from THP-1 Cells Differentially Modulate the Response of Cancer Cells to Etoposide. BMC Cancer. 2015, 15:577 20. Trombetta, A. C.; Soldano, S.; Contini, P.; Tomatis, V.; Ruaro, B.; Paolino, S.; Brizzolara, R.; Montagna, P.; Sulli, A.; Pizzorni, C.; Smith, V.; Cutolo, M. A Circulating Cell Population Showing Both M1 and M2 Monocyte/Macrophage Surface Markers Characterizes Systemic Sclerosis Patients with Lung Involvement. Respir. Res. 2018, 19:186 21. Mills, C. D.; Kincaid, K.; Alt, J. M.; Heilman, M. J.; Hill, A. M. M-1/M-2 Macrophages and the Th1/Th2 Paradigm. J. Immunol. 2000, $164,6166-6173$

22. Moon, H.; Lee, J.; Min, J.; Kang, S. Developing Genetically Engineered Encapsulin Protein Cage Nanoparticles as a Targeted Delivery Nanoplatform. Biomacromolecules. 2014, 15, 3794 - 3801

23. Lagoutte, P.; Mignon, C.; Stadthagen, G.; Potisopon, S.; Donnat, S.; Mast, J.; Lugari, A.; Werle, B. Simultaneous Surface Display and Cargo Loading of Encapsulin Nanocompartments and Their Use for Rational Vaccine Design. Vaccine. 2018, 36, 3622 - 3628

24. Greish, K. Enhanced Permeability and Retention (EPR) Effect for Anticancer Nanomedicine Drug Dargeting. Methods Mol. Biol. 2010, $624,25-37$

25. Rurup, W. F. Controlling the Loading of Protein Nanocages. Enschede 2013, Ch. 6, 93 - 105 
Chapter 5 


\section{Chapter 6}

\section{Exterior Modification of Thermotoga maritima}

Encapsulin with EETI-II Knottins

\section{Abstract}

A big hurdle for the use of protein based drugs is that they are easily degraded by proteases in the body. We demonstrate the possibility to functionalize Thermotoga maritima encapsulins with an mEETI-II knottin miniprotein from the cysteine stabilized knot class. The resulting particles did not show aggregation and retained part of their protease inhibition function. This resulted in protection from trypsin induced degradation of the protein cage. The used chemistry is easy to apply and thus suitable to protect other protein systems from degradation. Moreover, this proof of principle opens up the use of other knottins, which can be attached to protein cages to create a heterofunctionalized protein nanocage. This allows for example specific targeting and tumor suppression. Overall, this is a promising strategy to chemically modify a protein cage.

R. Klem, M. V. de Ruiter, J. J. L. M. Cornelissen; "Protecting Encapsulin Nanoparticles with Cysteine-Knot Miniproteins", Mol. Pharm., 2018, 15, 2991-2996. 


\subsection{Introduction}

Protein based formulations are increasingly used in drug applications due to their high efficiency and biocompatibility. They are applied as for example recombinant protein vaccines, ${ }^{1}$ as treatment for cancer ${ }^{2}$ and show promise for the treatment of other diseases. ${ }^{3}$ One of the major drawbacks of proteins as drugs is the reduced bioavailability, in part because these get degraded by proteases. To protect the proteins from degradation, they can be functionalized with polymers such as polyethylene glycol (PEG), which can increase efficacy but this still has various drawbacks related to the specificity of glycosylation. ${ }^{4}$ To address the issue of protease-based protein degradation more specifically, we seek to improve the stability of proteins using a modification strategy based on knottins.

Knottins are a class of miniproteins that are natural enzyme-inhibitor toxins. Their defined cysteine-stabilized knot structures result in a high thermal stability, protection from proteases ${ }^{5}$ and allow a great degree of freedom to modify their loops. ${ }^{6}$ The modified loop peptide sequence can be used for high affinity binding onto specific receptors and proteins, which exceeds the binding affinities of free peptides. Therefore, these short peptides hold great promise as stable and cheap antibody substitutes. A multitude of knottins and their derivatives have been developed or found in nature and are combined in a database. ${ }^{7,8}$ They function as analgesics, ${ }^{9,10}$ antimalarials ${ }^{11}$ and pesticides. ${ }^{12}$ Knottins are not immunogenic ${ }^{6}$ and can function in a variety of medical ways, such as anti-cancer drugs, anti-viral drugs and in vivo imaging. ${ }^{13,14}$

Knottins can also be used to inhibit specific proteins, such as trypsin, which is inhibited by the Ecballium elaterium trypsin inhibitor II (EETIII) knottin, which can be modified while maintaining its structure. ${ }^{15}$ The EETI-II knottins have three specific loops and one of these loops has a specific amino acid sequence that blocks the active site of trypsin using 
competitive inhibition. ${ }^{16}$ Sankaran et al. showed, using a kinetic analysis, that the inhibition is a mix of competitive and non-competitive inhibition. ${ }^{13}$ This inhibition has a $\sim 10 \mathrm{nM}$ affinity, ${ }^{17}$ which is caused by the specific amino acid sequence combined with the specific shape of the loop that is formed by the cysteines in the knottin. ${ }^{18}$ This makes EETI-II a suitable candidate for our study, given that trypsin is a main protease component of the digestive system of many vertebrates. Thus, trypsin is an important target in order to protect the protein of interest.

Protein-based nanocages are a class of protein materials that can benefit from such extra protection. These protein nanoparticles can function as biocompatible and biodegradable drug delivery platforms that can be used for the delivery of for example active small molecules, genes, ${ }^{19}$ proteins ${ }^{20}$ and nanoparticles. ${ }^{21}$ Protein cages include viruses, ferritins and recently discovered bacterial nano- and micro-compartments, including encapsulins. Encapsulins derived from Thermotoga maritima (TmE) are an especially interesting protein cage because of their native stability at high temperatures and over a wide $\mathrm{pH}$ range. TmE are icosahedral particles of $22 \mathrm{~nm}$ with a $\mathrm{T}=1$ symmetry, consisting of 60 capsid protein (CP) monomers, ${ }^{22}$ which can load foreign cargo. ${ }^{23}$ On each of these monomers one cysteine group is solvent exposed, allowing specific chemical modifications that can alter the encapsulin exterior and functionalize it in a controlled and defined manner. ${ }^{24}$

In this study, we genetically modified the EETI-II knottin to create a flexible and accessible N-terminus and we replaced the only lysine in the native knottin with a serine to avoid binding to other binding sites. We connected this knottin to TmE with a heterofunctional linker (EMCS) and created a functionalized protein nanocage with trypsin inhibitive properties. 


\subsection{Results and Discussion}

\subsubsection{Availability of Thiol-groups on the Tm Encap- sulin Exterior}

The production of TmE resulted in $\sim 20 \mathrm{~nm}$ sized nanoparticles as measured with DLS and FPLC (Figure 6.1). To verify that the thiol groups were available for binding, we reacted TmE with Oregon Green 488 maleimide-coupled dye. This was purified and subsequently analysed using UV-vis. Using the peak at $\lambda=488 \mathrm{~nm}$, which corresponds to the Oregon Green 488 absorption, and $\varepsilon=70,000 \mathrm{M}^{-1} \mathrm{~cm}^{-1}$, we determined the Oregon Green 488 concentration to be $2.49 \mu \mathrm{M}$ by using the LambertBeer law (Equation 6.1).

$$
A=\varepsilon * C * d
$$

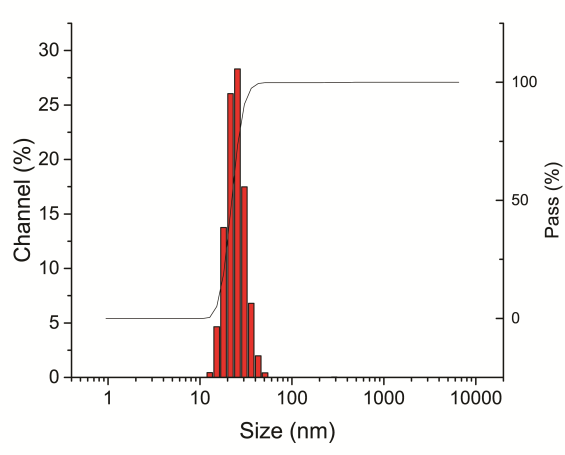

(a)

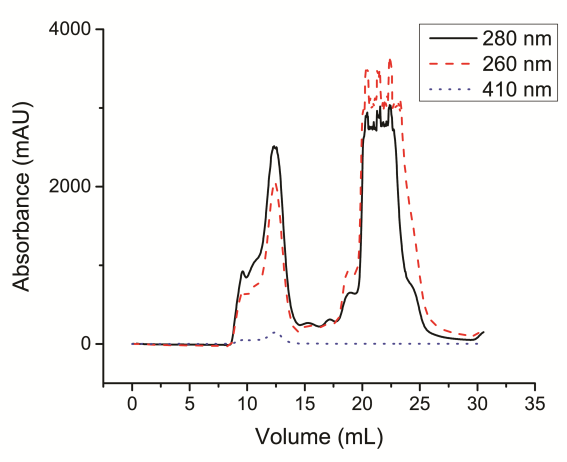

(b)

Figure 6.1: Structural analysis of TmE with A) DLS and B) FPLC. With FPLC, we collected fractions corresponding to the peak at $12.5 \mathrm{~mL}$.

The peak at $\lambda=280 \mathrm{~nm}$ corresponds to TmE which, when corrected for the Oregon Green 488 using a correction factor of 0.12 at $\lambda=280 \mathrm{~nm}$ and $\varepsilon=36,565 \mathrm{M}^{-1} \mathrm{~cm}^{-1}$, was used to calculate a TmE monomer concentration of $9.50 \mu \mathrm{M}$. This gives a degree of loading of $26.2 \%$, yielding 15 
dyes per encapsulin nanoparticle (Figure 6.2). This verified that the thiol groups on the exterior of TmE can be modified using maleimides. However, the binding efficiency of Oregon Green 488 could not be increased beyond $26 \%$, likely because the thiols from the cysteines that are accessible on the exterior, Cys 123, reside in a pocket ${ }^{22}$ and these might become hindered. This results in less accessible thiol groups. Additionally, because encapsulins are a dynamic system, even if the Oregon Green 488 dye enters the pocket, the orientation of the thiol group can be different, preventing the binding of the dye.

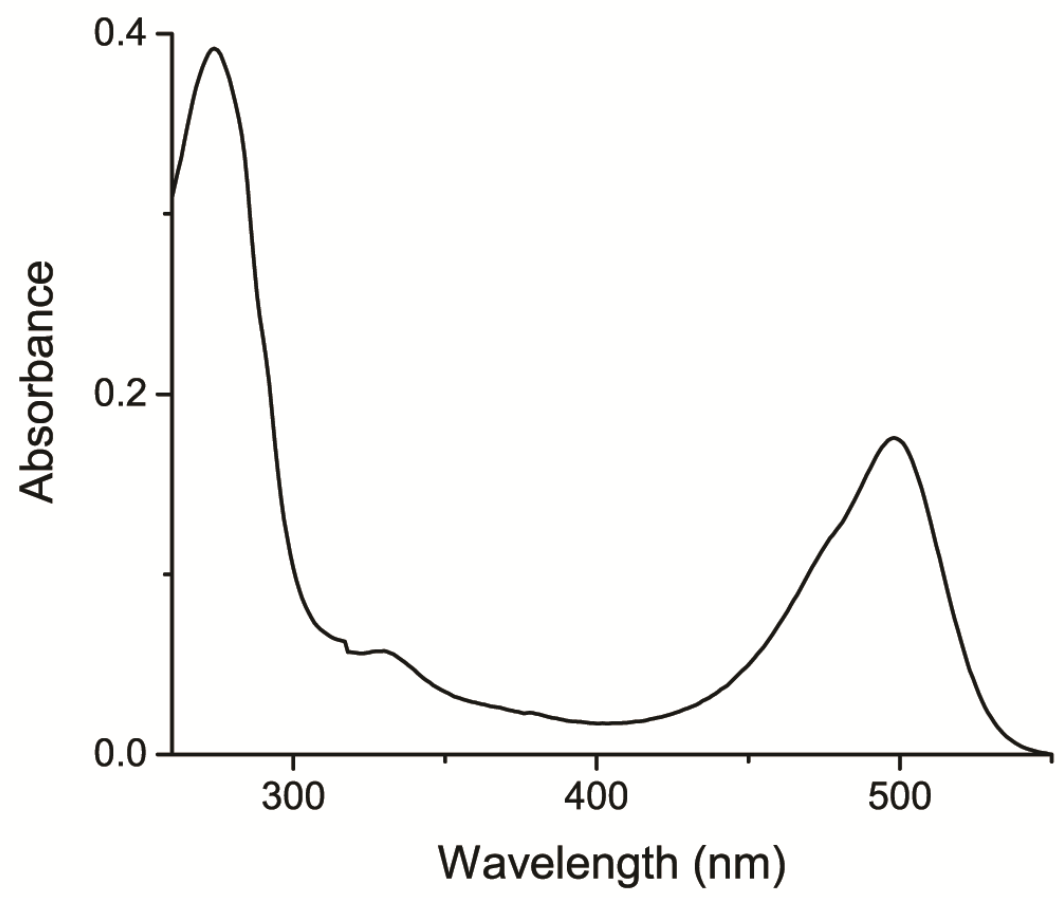

Figure 6.2: UV-vis spectrum of TmE with maleimide coupled Oregon Green 488 dye attached to the exterior thiol groups. 


\subsubsection{Production and Activity of EETI-II knottins}

To selectively bind the EETI-II knottin to TmE, we modified the EETI-II genetic sequence to replace a lysine from one of the loops with a serine (mEETI-II). This makes the N-terminus the only available binding site for bio-orthogonal binding with NHS-ester substitution. Furthermore, both the original and modified knottins where expressed in Escherichia coli with a flexible N-terminal linker. To verify that the knottins were successfully formed and produced, we performed MALDI-TOF. For EETI-II knottin, the expected weight was $\mathrm{M}=3,589.1 \mathrm{D}$ and the observed weight was $\mathrm{M}=3,590$ D. For mEETI-II, which was derived from EETI-II but with the replaced lysine, the expected weight was $\mathrm{M}=3,548 \mathrm{D}$ and the observed weight was $\mathrm{M}=3,548.9 \mathrm{D}$ (Figure 6.3).

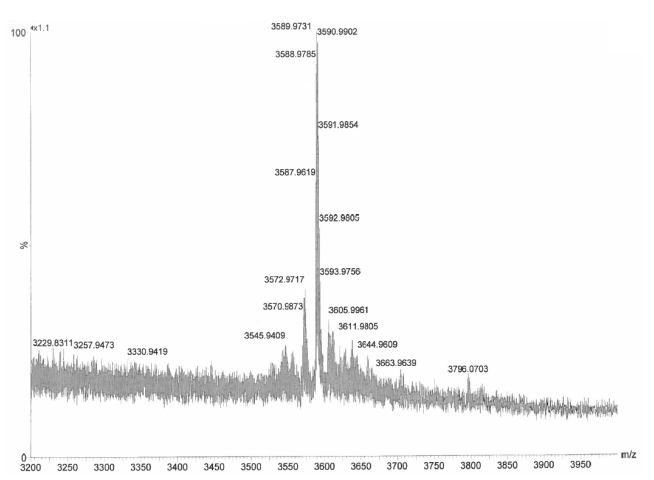

(a)

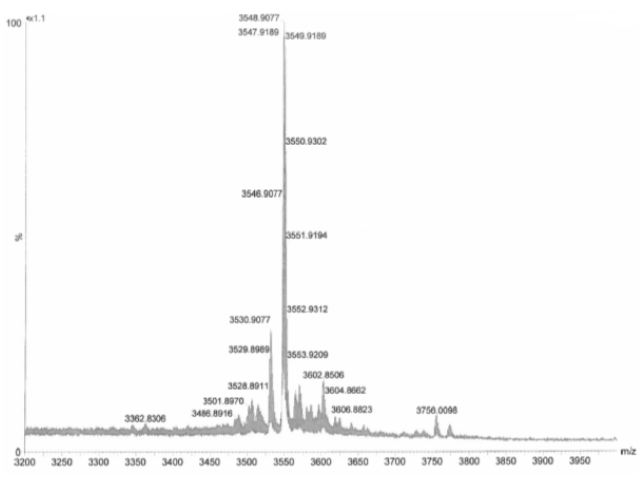

(b)

Figure 6.3: MALDI-TOF analysis of EETI-II knottin (A) and mEETI-II knottin (B). 


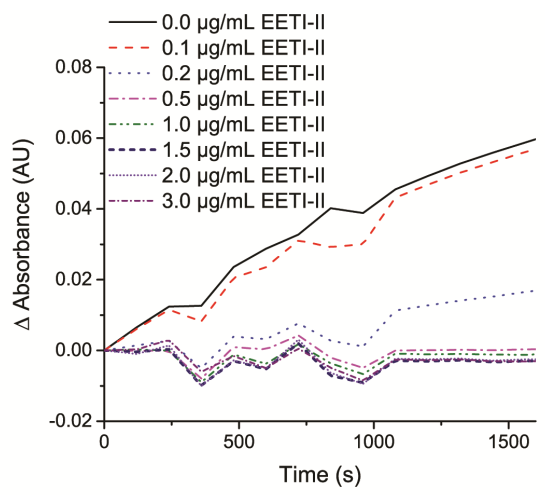

(a)

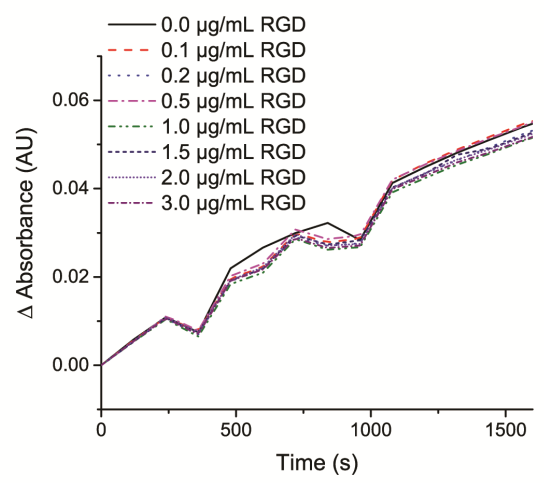

(c)

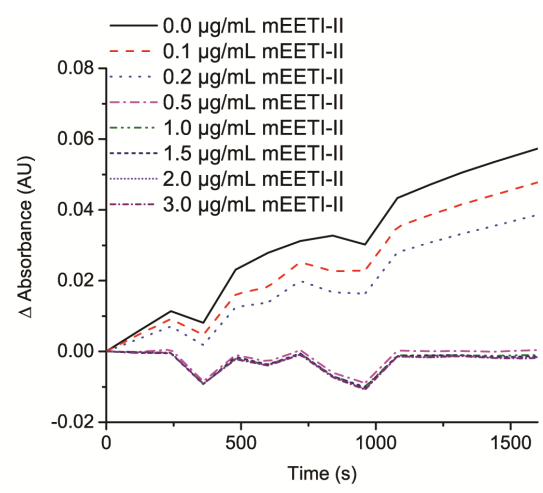

(b)

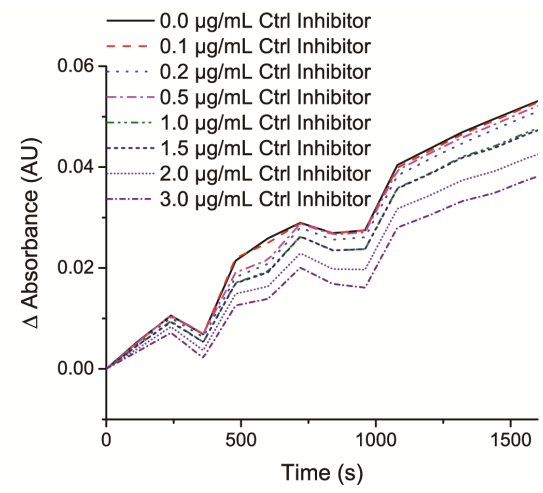

(d)

Figure 6.4: Differences in trypsin activity by A) EETI-II, B) mEETI-II, C) RGD-knottin and D) trypsin inhibitor from bovine pancreas. The $\mathrm{y}$-axis indicates the difference in absorbance compared with $\mathrm{t}=0$.

Thus, EETI-II knottins were correctly produced and altered to mEETI-II. To determine the inhibitory capabilities of the knottins, they were mixed with trypsin and added to the $\mathrm{N}_{a}$-Benzoyl-L-arginine 4-nitroanilide hydrochloride (L-BApNA) substrate. Upon cleavage by trypsin, L-BApNA forms para-nitroaniline and the absorption at $\lambda=405 \mathrm{~nm}$ increases. The mEETI-II knottin shows similar trypsin inhibition compared to the EETIII knottins (Figure $6.4 \mathrm{~A}$ and B). The control sample (RGD knottin) ${ }^{26}$ did not inhibit trypsin (Figure $6.4 \mathrm{C}$ ), while the trypsin inhibitor from bovine pancreas showed minor trypsin inhibition (Figure 6.4D). 


\subsubsection{Binding and Activity of Knottins to Thermo- toga maritima Encapsulin}

We connected EMCS to the amine group of the mEETI-II knottins and reacted this to the TmE. The mEETI-II knottins cannot be distinguished with UV spectroscopy when attached to the protein nanocage - both absorb at $\lambda=280 \mathrm{~nm}$. The change in extinction coefficient $(\varepsilon)$ of the knottins (with the added tryptophan) is not significant compared with the $\varepsilon$ of 60 encapsulin monomers. Therefore, the main method of detection is by monitoring the trypsin inhibition capacity of the knottins (comparable to the experiments demonstrated in Figure 6.4). We found that TmE, without functionalization with knottins, already inhibit trypsin activity.

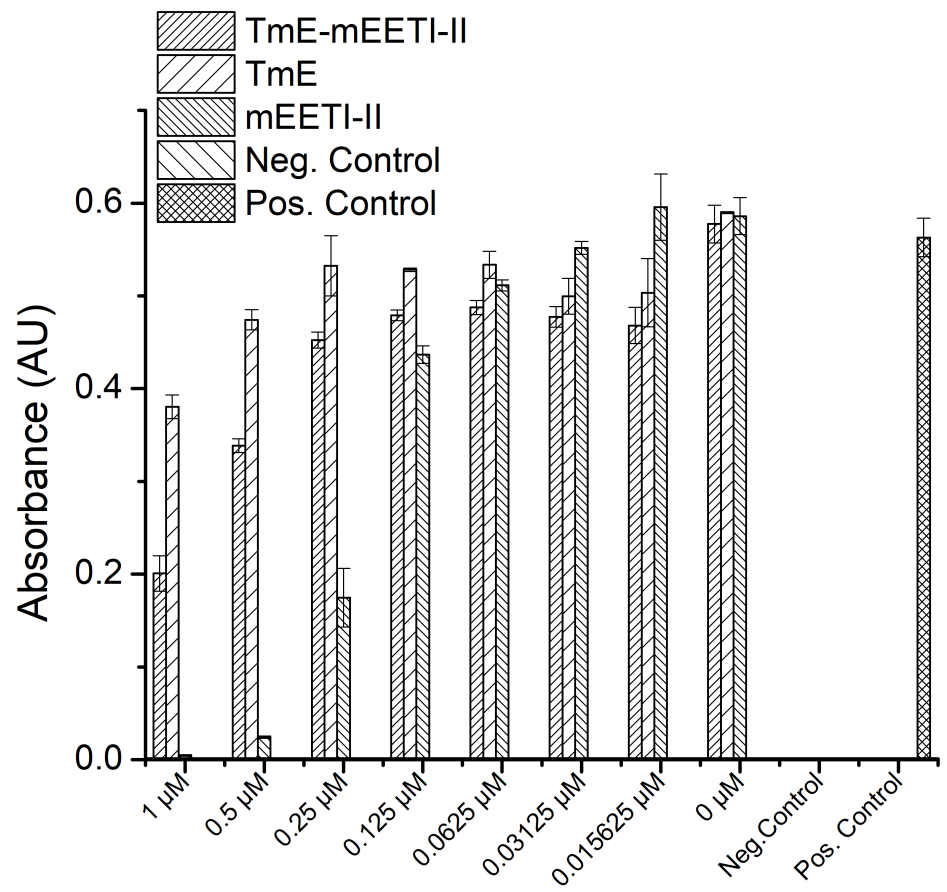

Figure 6.5: Absorbance at $405 \mathrm{~nm}$ which is present when l-BApNA is cleaved by trypsin. The $\mathrm{x}$-axis shows the concentrations of the different inhibitors. All measurements were performed after $1 \mathrm{~h}$ incubation. The error bars indicate the standard deviation $(\mathrm{N}=3)$. 
Compared to TmE, TmE-mEETI-II showed enhanced but not complete inhibition within the concentration range studied but not as significant as unbound mEETI-II. This is probably caused by steric hindrance of the knottins by the encapsulin complex; the binding of a complex to knottins is known to decrease the knottin activity. ${ }^{13}$ After $1 \mathrm{~h}$ and at $1 \mu \mathrm{M}$, mEETI-II fully inhibits trypsin, TmE inhibits $32.43 \%$ and TmE-mEETIII inhibits $64.33 \%$. At $0.5 \mu \mathrm{M}$, the difference between functionalized and non-functionalized TmE is still significant $(\mathrm{P} \leq 0.001$ determined with an unpaired t-test) (Figure 6.5). At lower concentrations, the effects of TmE, either functionalized or not, are not significantly different from the positive control.

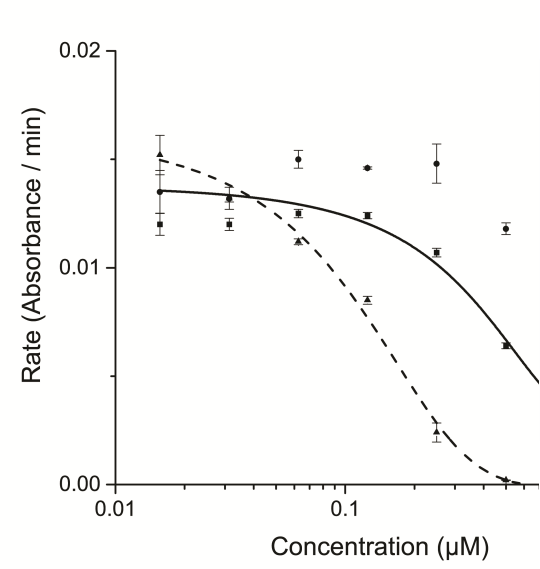

(a)

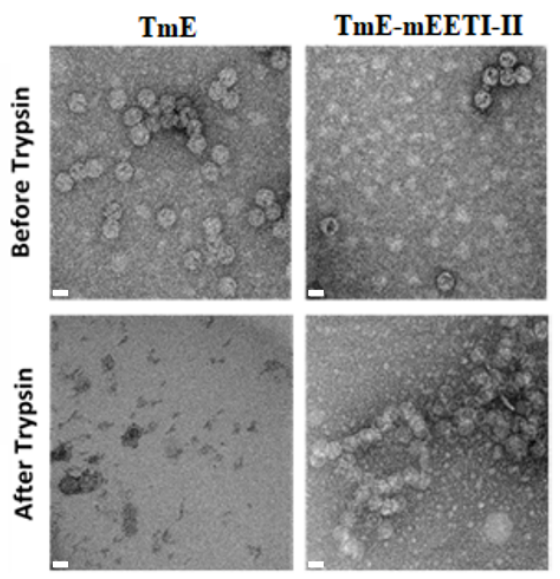

(b)

Figure 6.6: A) The rate of l-BApNA cleavage by trypsin in the presence of TmE $(\bullet)$, mEETI-II $(\boldsymbol{\Delta})$ and TmE-mEETI-II (ם). B) TEM images of TmE encapsulins both protected and unprotected by mEETI-II knottins, both before and after trypsin treatment. Scalebars represent $20 \mathrm{~nm}$. 
We determined the rate of conversion based on the absorbance at $\lambda=405$ $\mathrm{nm}$ using the slope in the linear range $(\mathrm{t}=0-20 \mathrm{~min})$. These data were fitted with a sigmoidal fit with a dose response function using OriginPro 9.1 (Figure 6.6A). The $\mathrm{EC}_{50}$ of each compound was determined using this fit; the $\mathrm{EC}_{50}$ for mEETI-II was $0.13 \mu \mathrm{M}$, for TmE-mEETI-II $0.73 \mu \mathrm{M}$ and for $\mathrm{TmE}$ the $\mathrm{EC}_{50}$ was $\geq 1 \mu \mathrm{M}$, but could not be fitted to a sigmoid curve.

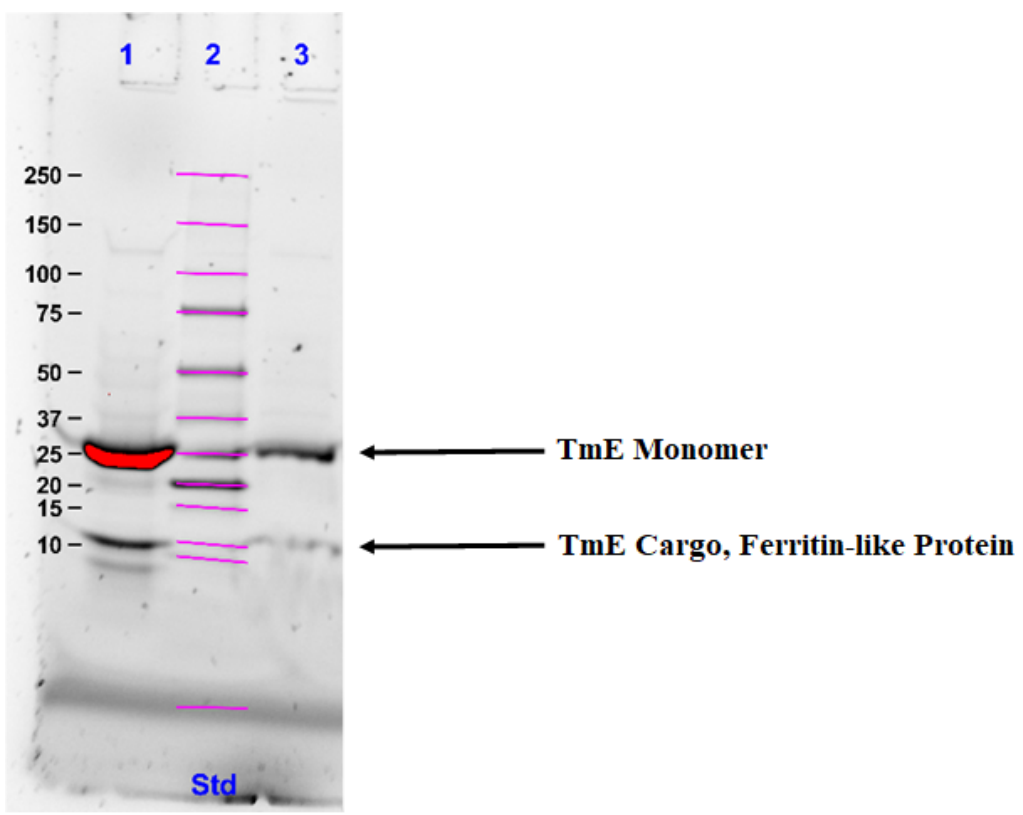

Figure 6.7: SDS-PAGE of TmE and mEETI-II before mixing (lane 1), ladder (lane 2) and TmE-mEETI-II (lane 3). The TmE monomers have a mass of $\sim 30 \mathrm{kDa}$ (determined using ExPASy ProtParam). The TmE monomers and the native TmE cargo (ferritin-like protein) are highlighted by the arrows. We could not observe a difference between TmE monomers that did and did not have an mEETI-II knottin attached to it due to the small size of the knottin compared to the TmE monomer $(3 \mathrm{kDa}$ and 30 $\mathrm{kDa}$, respectively). Other assays were also not sufficient to quantify the amount of knottins bound to TmE. 
Equipping TmE with mEETI-II does not change the structure of the protein cages (Figure 6.6B). Both native TmE and the TmE-mEETI-II were treated with trypsin, and with the TmE we found a complete loss of the structural integrity. The TmE-mEETI-II did aggregate, but kept the individual cage structure (Figure 6.6B).

We performed SDS-PAGE to verify that no inhibition was caused by unbound mEETI-II in the TmE-mEETI-II sample. The filtration steps were sufficient to remove unbound mEETI-II from TmE encapsulins, given the absence of a band at $3.5 \mathrm{kDa}$ in the SDS-PAGE experiments (Figure 6.7).

\subsubsection{Discussion}

In this study we used TmE as a model to functionalize protein nanocages with EETI-II knottins. TmE can be functionalized specifically by binding compounds to the exterior cysteines, which we show are available for chemical binding (Figure 6.2). Sonotaki et al. have PEGylated Rhodococcus erythropolis N771 encapsulins demonstrating exterior modification. ${ }^{27}$ Specific functionalization has not been studied. Moon et al. genetically engineered a targeting loop in TmE, ${ }^{24,28}$ but this method might be limited because they altered the monomers forming the encapsulin shell.

We chemically modified the encapsulins with the trypsin inhibiting knottin EETI-II. The knottin required modification to prevent non-specific binding. The native EETI-II knottin contains a lysine ${ }^{15}$ that could bind the EMCS linker and connect the knottin to the encapsulin in such a way that the active loop cannot be accessed. Therefore, we modified the EETI-II knottin to replace the lysine with a non-reactive serine and extend the N-terminus with an SGSGS-sequence to increase the length between the knottin and the encapsulin surface. As shown using the trypsin inhibition assay, these modifications were successful and did not affect the knottin activity (Figure 6.3 and Figure 6.4A and B). 
Since EETI-II consists of 35 amino acids but contains no phenylalanine, tyrosine or tryptophan, UV-vis detection is not possible. Thus, we added a tryptophan to the C-terminus to both EETI-II and mEETI-II. This allowed for UV-vis detection when the knottin was cleaved from the teal fluorescent protein (TFP). However, when bound to TmE, which consists of 60 monomers of 268 amino acids, the single tryptophan is not sufficient to detect the presence of knottins attached to encapsulins. This makes it impossible to use UV-vis absorption to determine the number of knottins attached to one TmE.

To study this, the biological activity of EETI-II was used to determine if it was bound to TmE. However, this proved to be non-conclusive. Nonfunctionalized encapsulins have an inhibitory effect on trypsin (Figure 6.6A and Figure 6.3), probably caused by competition between TmE and L-BApNA as substrate for trypsin. This $\mathrm{EC}_{50}$ is significantly lower than TmE functionalized with mEETI-II. Here, the TmE show a significantly increased inhibition, which we attribute to the presence of mEETI-II, but they do not achieve similar $\mathrm{EC}_{50}$ as mEETI-II alone. Potentially, the bulk of the TmE limits the inhibitory effect of mEETI-II. This issue can potentially be overcome by using a longer linker, which results in a more available knottin. But the data do suggest that the mEETI-II knottins are bound to TmE and increase trypsin inhibition and thus TmE stability.

The hypothesis that mEETI-II functionalized TmE are more stable is strengthened further by Transmission Electron Microscopy (TEM) analyses. These show that TmE are degraded when trypsin is added, while TmE-mEETI-II keep their individual morphological structure, but aggregates are formed (Figure 6.6B). This could be a result of interactions between the TmE-mEETI-II-trypsin complexes, or hydrophobic interactions between TmE. The structural origin of this aggregation requires further investigation. 
The chemical modification of TmE via the thiol groups is also demonstrated and can be expanded beyond the use of knottins. Other molecules, such as PEG, targeting moieties ${ }^{29}$ and tumor suppressors ${ }^{30}$ could be added chemically to the TmE. Additionally, other reactive groups, including the amines and carboxylic acid groups from the lysines and ascorbic and glutamic acids, respectively, could be utilized for to create heterofunctionally modified encapsulins.

\subsection{Conclusions}

In conclusion, we show the possibility to functionalize a protein, in this case the Thermotoga maritima encapsulin nanoparticle, with mEETI-II knottins. We can modify these knottins without affecting their activity and use them to protect TmE from trypsin induced degradation. This concept can be applied to other nanocages and proteins to protect them from trypsin degradation or add a new functionality.

\subsection{Acknowledgements}

I thank dr. M. V. de Ruiter for producing the knottins and his collaboration during the various assays and $\mathrm{R}$. van der Hee for her help with the MALDI-TOF experiments. I thank dr. E. G. Keim for his assistance with the TEM measurements. 


\subsection{Experimental Section}

\subsubsection{Materials}

Chemicals were purchased from Sigma-Aldrich unless stated otherwise. Milli-Q water was obtained by ultrafiltration (Millipore Adv. A10, 18 $\mathrm{M} \Omega \mathrm{cm}$ at $\left.25^{\circ} \mathrm{C}\right)$.

\subsubsection{Equipment}

UV-vis measurements to determine protein and DNA concentrations were performed using a Thermo Fisher Scientific Nanodrop 1000 and a Perkin Elmer Lambda 850 spectrometer. Standard quartz cuvettes with a $1 \mathrm{~cm}$ path length were used. Kinetic absorption measurements for the trypsin inhibitor assays were performed using a TECAN infinite 200 pro plate reader. For TEM analysis samples $(5 \mu \mathrm{L})$ were applied onto Formvarcarbon coated grids. After $1 \mathrm{~min}$, the excess of liquid was drained using filter paper. Uranyl acetate $(5 \mu \mathrm{L}, 1 \% \mathrm{w} / \mathrm{v})$ was added and the excess of liquid was drained after $15 \mathrm{sec}$ and the samples were dried for $30 \mathrm{~min}$ at room temperature. Imaging was performed on an FEG-TEM (Phillips $\mathrm{CM}$ 30) operated at $300 \mathrm{kV}$ acceleration voltages. Dynamic light scattering (DLS) measurements were performed using a Microtrac Nanotrac Wave W3043. The viscosity and refractive index of water and the refractive index of proteins (1.54) were used. Matrix assisted laser desorption/ionization time-of-flight (MALDI-TOF) analysis was performed with a Waters MALDI SYNAPT G1 high definition mass spectrometer on a sinapic acid matrix. 


\subsubsection{Purification of Thermotoga maritima Encap- sulin}

TmE were produced and purified using the method described by Rurup et al..$^{25}$ and in the experimental section of Chapter 3. There were slight modifications to these protocols to accomodate the fact that these were different encapsulins. The protocol for the purification of TmE differs slightly from that of Brevibacterium linens encapsulins. The sample was ultracentrifuged after RNAse addition at $234,000 \mathrm{x} \mathrm{g}, 17 \mathrm{~h}, 10^{\circ} \mathrm{C}$, and subsequently dissolved the pellet in $1 \mathrm{~mL}$ encapsulin buffer $(20 \mathrm{mM}$ Trizma base, $150 \mathrm{mM} \mathrm{NH}_{4} \mathrm{Cl}, 20 \mathrm{mM} \mathrm{MgCl}_{2}, 1 \mathrm{mM} \beta$-mercaptoethanol, $\mathrm{pH} 7.5)$.

\subsubsection{Fluorophore Binding on Thermotoga maritima Encapsulin}

TmE were rebuffered to $0.1 \mathrm{x}$ PBS buffer with $0.1 \%$ (v/v) TWEEN-20 using Zeba spin 7k MWCO desalting columns (Thermo Fisher). Oregon Green 488-maleimide dye was dissolved in DMSO at $1 \mathrm{mg} / \mathrm{mL}$ and mixed with TmE in a 1:120 ratio (encapsulin to Oregon Green 488). The reaction was incubated for $1 \mathrm{~h}$ at $21^{\circ} \mathrm{C}$ and purified twice using the Zeba spin column.

\subsubsection{Molecular Cloning of (m)EETI-II Constructs}

The molecular cloning of (m)EETI-II constructs was performed similar to a protocol described by Sankaran et al., ${ }^{13}$ but with several modifications. ssDNA sequences corresponding to the $\beta$-trypsin inhibitor knottins were ordered from Eurofins MWG Operon, Germany. The 3'-terminal contained a stop codon. 
The following genetic sequence was used for EETI-II:

GAATCCGGCTCCGGCTCCGGCTGCCCGCGTATTCTGATGCGTT GCAAACAGGATTCCGATTGCCTGGCGGGCTGCGTGTGCGGCC CGAATGGCTTTTGCGGCTGGTAA

and for mEETI-II:

GAATCCGGCTCCGGCTCCGGCTGCCCGCGTATTCTGATGCGTT GCTCCCAGGATTCCGATTGCCTGGCGGGCTGCGTGTGCGGCC CGAATGGCTTTTGCGGCTGGTAA.

The SGSGS-spacer is highlighted in blue, the lysine which was changed to serine in bold and the added C-terminal tryptophan in red. The genetic constructs had 5' BsrGI and 3' NheI restriction sites, which were used to insert the gene behind the gene for TFP through restriction digestion enzymes on the suppliers' and the pET-15b plasmid. The samples were purified with agarose gel electrophoresis, using Wizard ${ }^{\circledR}$ SV Gel and PCR Clean-Up System (Promega) followed by ligation with T4 DNA ligase (New England Biolabs Inc.). The resulting pET15b TFP-knottin plasmids were transformed to NovaBlue ultracompetent cells (Novagen) and grown overnight on LB agar plates containing $100 \mathrm{mg} / \mathrm{L}$ ampicillin. Plasmids were extracted from individual colonies using a Qiagen spin miniprep kit and sequenced by Eurofins using a standard T7 terminal reverse primer. Afterwards, the two different plasmids were transfected into Rosetta Gami (DE3) pLysS competent cells (Novagen) and grown overnight on LB agar plates containing $34 \mathrm{mg} / \mathrm{L}$ chloramphenicol and $100 \mathrm{mg} / \mathrm{L}$ ampicillin. Individual colonies were grown in LB medium containing the mentioned antibiotics. For long-term storage, $15 \%$ glycerol bacterial stocks were made, which were stored at $-80^{\circ} \mathrm{C}$. 


\subsubsection{Expression and Purification of ( $\mathrm{m})$ EETI-II Knottins}

The expression and purification of EETI-II and mEETI-II knottins was performed according to the protocol by Sankaran et al. ${ }^{13}$ As an alternative to the dialysis purification, size exclusion liquid chromatography (SEC) was performed using an ÄKTA purifier with a Frac-950 fractionation collector (GE Healthcare Life Sciences) equipped with a Superdex 75 10/300GL (GE Healthcare Life Sciences) column. Both methods yielded comparable results. Concentrations of the knottins were determined from the absorbance $\lambda=280 \mathrm{~nm}$ with an $\varepsilon=5,875 \mathrm{M}^{-1} \mathrm{~cm}^{-1}$.

\subsubsection{Binding of (m)EETI-II Knottins to Thermo- toga maritima Encapsulin}

The knottins were mixed with a freshly prepared batch of sulfo-EMCS (N$\varepsilon$-malemidocaproyl-sul-fosuccinimide ester, Thermo Fisher) solution at a 50x molar excess for $1 \mathrm{~h}$ at room temperature. The unreacted sulfo-EMCS and side products were removed from the knottins using 3k MWCO centrifugal filters (Amicon Ultra) with 3x buffer refreshment (0.1x PBS, 0.1\% (v/v) TWEEN-20). TmE was rebuffered to the same buffer using Zeba spin 7k MWCO desalting columns (Thermo Fisher). The concentrations of both knottins and encapsulins were measured with a nanodrop system and the EMCS-knottins were reacted to the encapsulin at a 100x molar excess for at least $1 \mathrm{~h}$ at $21^{\circ} \mathrm{C}$. The unbound knottins were removed by applying a Zeba spin 40k MWCO desalting column on the TmE-mEETIII solution twice. 


\subsubsection{SDS-PAGE Analysis of Trypsin Digestion of Thermotoga maritima Encapsulin}

Fifteen $\mu \mathrm{L}$ TmE, either with or without mEETI-II knottins attached to it, were added to $15 \mu \mathrm{L}$ of a 50/50 mixture of $\beta$-mercaptoethanol and Laemmli sample buffer (Bio-Rad). The mixtures were incubated at $99^{\circ} \mathrm{C}$ for $5 \mathrm{~min}$ to denature the proteins. The samples were analysed with SDS-PAGE on a $4-20 \%$ gel (Bio-Rad). The unstained ladder was 10 $\mu \mathrm{L}$ of Precision Plus Protein ${ }^{\mathrm{TM}}$ Standard (Bio-Rad). Electrophoresis was conducted at $100 \mathrm{~V}$ for $1 \mathrm{~h}$. Gels where activated with UV-light for 5 min on a stain-free enabled UV transilluminator and imaged with a Gel Doc $^{\mathrm{TM}}$ EZ system with Image Lab software (Bio-Rad).

\subsubsection{Trypsin Assay}

TmE were rebuffered to $0.1 \mathrm{x}$ PBS $+0.05 \%$ (v/v) TWEEN 20 with $7 \mathrm{k}$ MWCO Zeba Spin columns. The samples were added to a flat-bottom 96-wells plate (Greiner) in triplo to a final concentration ranging from 0 - $1 \mu \mathrm{M}$. Trypsin was added to a final concentration of $0.1 \mathrm{mg} / \mathrm{mL}$. Immediately prior to analysis $10 \mu \mathrm{l}$ L-BApNA was added (final concentration $142.2 \mu \mathrm{g} / \mathrm{mL})$. We measured the absorbance at $\lambda=405 \mathrm{~nm}$. 


\subsection{References}

1. Nascimento, I. P.; Leite, L. C. C. Recombinant Vaccines and the Development of New Vaccine Strategies. Braz. J. Med. Biol. Res. 2012, 45, 1102-1111

2. Pieters, R.; Hunger, S. P.; Boos, J.; Rizzari, C.; Silverman, L.; Baruchel, A.; Goekbuget, N.; Schrappe, M.; Pui, C. L-asparaginase Treatment in Acute Lymphoblastic Leukemia: a Focus on Erwinia Asparaginase. Cancer. 2011, 117, 238-249

3. Kirkegaard, T.; Gray, J.; Priestman, D. A.; Wallom, K. L.; Atkins, J.; Olsen, O. D.; Klein, A.; Drndarski, S.; Petersen, N. H.; Ingemann, L.; Smith, D. A.; Morris, L.; Bornæs, C.; Jørgensen, S. H.; Williams, I.; Hinsby, A.; Arenz, C.; Begley, D.; Jäättelä, M.; Platt, F. M. Heat Shock Protein-based Therapy as a Potential Candidate for Treating the Sphingolipidoses. Sci. Transl. Med. 2016, 8, 355

4. Solá, R. J.; Griebenow, K. Glycosylation of Therapeutic Proteins: An Effective Study to optimize Efficacy. BioDrugs. 2010, 24, 9-21

5. Getz, J. A.; Rice, J. J.; Daugherty, P. S. Protease-resistant Peptide Ligands from a Knottin Scaffold Library. ACS Chem. Biol. 2011, 6, 837-844

6. Kolmar, H Alternative Binding Proteins: Biological Activity and Therapeutic Potential of Cystine-knot Miniproteins. FEBS J. 2008, 275, 2684-2690

7. Gelly, J. C.; Gracy, J.; Kaas, Q.; Le-Nguyen, D.; Heitz, A.; Chiche,

L. The KNOTTIN Website and Database: a New Information System Dedicated to the Knottin Scaffold. Nucleic Acids Res. 2004, 32, D156D159

8. Gracy, J.; Le-Nguyen, D.; Gelly, J. C.; Kaas, Q.; Heitz, A.; Chiche, L. KNOTTIN: the Knottin or Inhibitor Cystine Knot Scaffold in 2007. Nucleic Acids Res. 2008, 36, D314-D319 
9. Souza, A. H.; Ferreira, J.; Cordeiro Mdo, N.; Vieira, L. B.; De Castro, C. J.; Trevisan, G.; Reis, H.; Souza, I. A.; Richardson, M.; Prado, M. A.; Prado, V. F.; Gomez, M. V. Analgesic Effect in Rodents of Native and Recombinant Ph Alpha 1beta Toxin, a High-voltage-activated Calcium Channel Blocker Isolated from Armed Spider Venom. Pain. 2008, $140,115-126$

10. Park, S. P.; Kim, B. M.; Koo, J. Y.; Cho, H.; Lee, C. H.; Kim, M.; Na, H. S.; Oh, U. A Tarantula Spider Toxin, GsMTx4, Reduces Mechanical and Neuropathic Pain. Pain. 2008, 137, 208-217

11. Choi, S. J.; Parent, R.; Guillaume, C.; Deregnaucourt, C.; Delarbre, C.; Ojcius, D. M.; Montagne, J. J.; Célérier, M. L.; Phelipot, A.; Amiche, M.; Molgo, J.; Camadro, J. M.; Guette, C. Isolation and Characterization of Psalmopeotoxin I and II: Two Novel Antimalarial Peptides from the Venom of the Tarantula Psalmopoeus Cambridgei. FEBS Lett. 2004, 572, 109-117

12. Gould, A.; Ji, Y.; Aboye, T. L.; Camarero, J. A. Cyclotides, a Novel Ultrastable Polypeptide Scaffold for Drug Discovery. Curr. Pharm. Des. 2011, 17, 4294-4307

13. Sankaran, S.; de Ruiter, M. V.; Cornelissen J. J. L. M.; Jonkheijm, P. Supramolecular Surface Immobilization of Knottin Derivatives for Dynamic Display of High Affinity Binders. Bioconjug. Chem. 2015, 26, 1972-1980

14. Moore, S. J.; Leung, C. L.; Cochran, J. R. Knottins: Disulfidebonded Therapeutic and Diagnostic Peptides. Drug Discov. Today Technol. 2012, 9, e1-e70

15. Kintzing, J. R.; Cochran, J. R. Engineered Knottin Peptides as Diagnostics, Therapeutics, and Drug Delivery Vehicles. Curr. Opin. Chem. Biol. 2016, 34, 143-150 
16. Bode, W., Greyling, H. J., Huber, R., Otlewski, J., and Wilusz, T. The Refined 2.0 A X-ray Crystal Structure of the Complex Formed Between Bovine beta-trypsin and CMTI-I, a Trypsin Inhibitor from Squash Seeds (Cucurbita maxima). Topological Similarity of the Squash Seed Inhibitors with the Carboxypeptidase A Inhibitor from Potatoes. FEBS Lett. 1989, 242, 285-292.

17. Krätzner, R.; Debreczeni, J. E.; Pape, T.; Schneider, T. R.; Wentzel, A.; Kolmar, H., Sheldrick, G. M.; Uson, I. Structure of Ecballium Elaterium Trypsin inhibitor II (EETI-II): a Rigid Molecular Scaffold. Acta Crystallogr. D. Biol. Crystallogr. 2005, 61, 1255-1262

18. Schmoldt, H-U.; Wentzel, A.; Becker, S.; Kolmar, H. A Fusion Protein System for the Recombinant Production of Short Disulfide Bond Rich Cystine Knot Peptides Using Barnase as a Purification Handle.Prot. Expr. And Pur. 2005, 39, 82-89

19. Azizgolshani, O.; Garmann, R. F.; Cadena-Nava, R.; Knobler, C. M.; Gelbart, W. M. Reconstituted Plant Viral Capsids can Release Genes to Mammalian Cells. Virology. 2013, 441, 12-17

20. O'Neil, A.; Reichhardt, C.; Johnson, B.; Prevelige, P. E.; Douglas, T. Genetically Programmed in Vivo Packaging of Protein Cargo and its Controlled Release from Bacteriophage P22. Angew. Chem. Int. Ed. Engl. 2011, 50, 7425-7428

21. Yang, Y.; Burkhard, P. Encapsulation of Gold Nanoparticles into Self-assembling Protein Nanoparticles. J. Nanobiotechnology. 2012, 10 22. Sutter, M.; Boehringer, D.; Gutmann, S.; Günther, S.; Loessner, M. J.; Prangishvili, D.; Stetter, K. O.; Weber-Ban, E.; Ban, N. Structural Basis of Enzyme Encapsulation into a Bacterial Nanocompartment. Nat. Struct. Mol. Biol. 2008, 15, 939-947

23. Cassidy-Amstutz, C.; Oltrogge, L.; Going, C. C.; Lee, A.; Teng, P.; Quintanilla, D.; East-Seletsky, A.; Williams, E. R.; Savage, D. F. Identification of a Minimal Peptide Tag for in Vivo and in Vitro Loading of Encapsulin. Biochemistry. 2016, 55, 3461-3468 
24. Moon, H.; Lee, J.; Min, J.; Kang, S. Developing Genetically Engineered Encapsulin Protein Cage Nanoparticles as a Targeted Delivery Nanoplatform. Biomacromolecules. 2014, 15, 3794-3801

25. Rurup, W. F.; Snijder, J.; Koay, M. S.; Heck, A. J.; Cornelissen, J. J. L. M. Self-sorting of Foreign Proteins in a Bacterial Nanocompartment. J. Am. Chem. Soc. 2014, 136, 3828-3832

26. Sankaran, S.; Cavatorta, E.; Huskens, J.; Jonkheijm, P. Cell Adhesion on RGD-Displaying Knottins with Varying Numbers of Tryptophan Amino Acids to Tune the Affinity for Assembly on Cucurbit[8]uril Surfaces. Langmuir. 2017, 33, 8813-8820

27. Sonotaki, S.; Takami, T.; Noguchi, K.; Odaka, M.; Yohda, M.; Murakami, Y. Successful PEGylation of Hollow Encapsulin Nanoparticles from Rhodococcus Erythropolis N771 without Affecting Their Disassembly and Reassembly Properties. Biomater. Sci. 2017, 5, 1082-1089

28. Moon, H.; Lee, J.; Kim, H.; Heo, S.; Min, J.; Kang, S. Genetically Engineering Encapsulin Protein Cage Nanoparticle as a SCC-7 Cell Targeting Optical Nanoprobe. Biomater. Sci. 2014, 18, 21

29. Kimura, R. H.; Cheng, Z.; Gambhir, S. S.; Cochran, J. R. Engineered Knottin Peptides: A New Class of Agents for Imaging Integrin Expression in Living Subjects. Cancer Res. 2009, 69, 2435-2442 30. Treggiari, D.; Zoccatelli, G.; Molesini, B.; Degan, M.; Rotino, G. L.; Sala, T.; Cavallini, C.; MacRae, C. A.; Minuz, P.; Pandolfini, T. A Cystine-knot Miniprotein from Tomato Fruit Inhibits Endothelial Cell Migration and Angiogenensis by Affecting Vascular Endothelial Growth Factor Receptor (VEGFR) Activation and Nitric Oxide Production. Mol. Nutr. Food Res. 2015, 59, 2255-2266 
Exterior Modification of Thermotoga maritima Encapsulin with EETI-II

Knottins 
Chapter 6

6 


\section{Summary}

Encapsulins are protein nanocages whose discovery by Sutter et al. has created potential for a new area of study. While no applications have been studied yet, the potential of encapsulins is evident from studies demonstrating their potential to encapsulate different proteins and to use encapsulins to transport therapeutic particles, such as dyes and metals. From this foundation, that is described in Chapter 2, we explored the possibilities of encapsulins in the following chapters.

This starts with the characterization of encapsulins. While the structure of Thermotoga maritima encapsulin (TmE) was known and while there is significant genetic similarity between different encapsulins, we found that the structure of Brevibacterium linens (BlE) is slightly different. Using cryo-EM we were able to create a near atomic resolution map of the $\sim 20 \mathrm{~nm}$ particle, which is described in Chapter 3. Here we found that while the fundamental structure of BlE likely consists of dimers, trimers can also be observed. Additionally, the E-loop appears to affect the size and structure of the encapsulins. Furthermore, we recognized loops on the exterior of $\mathrm{BlE}$ that can be modified to connect various functional molecules. While we did not place new exterior modifications on the BlE, we were able to chemically modify TmE with a trypsin inhibitor (Chapter 6 ). This demonstrated the potential to modify encapsulins and give them additional functionality, which can be expanded with for example targeting moieties, diagnostic tools and stabilizers. 
This modification was not limited to the exterior of encapsulins. It was already demonstrated that encapsulins can accommodate non-native proteins, but medically relevant cargo has not been studied. In Chapter 4, we demonstrate the modification of BlE with L-asparaginase (ASNase). The enzyme remained active, although its activity was decreased. We hypothesize that this is due to the encapsulin shell being dense, as demonstrated in the structural analysis in Chapter 3, limiting transport of the product (L-aspartate). While this dense shell provides the protection for its cargo (Chapter 5), it also affects the potential enzyme cargo. We also found that the encapsulins with ASNase had a biological effect; acute lymphoblastic leukemia cells died because of the BlE-ASNase. However, this effect was not as pronounced as with the native ASNase and the ASNase encapsulated in the more porous CCMV-based capsid. Potentially, different enzymes for other medical purposes can be created and studied.

For medical applications a detailed understanding of their interactions with cells is essential. This domain of encapsulins had not been studied sufficiently before. In Chapter 5, initial studies on the internalization of encapsulins and their potential immunogenicity are discussed. We found that not all cells internalize encapsulins; both HeLa and C2C12 cells either did not internalize encapsulins, or did so at a slower rate and only at high encapsulin concentrations, while macrophages do internalize encapsulins. This demonstrates the potential that encapsulins have in targeted drug delivery. Their native uptake in non-macrophage cells seems to be very low, which would result in reduced side effects if encapsulins are used for therapy. 
Another potential issue is immunogenicity, as we did find M1 differentiation in RAW264.7 macrophages that were treated with encapsulins. This would reduce efficiency of encapsulins as a drug delivery system, but could be beneficial if encapsulins are used for vaccine delivery.

We also found that the internal cargo (in our case mTFP) was not degraded by the macrophages even $48 \mathrm{~h}$ after incubation after being released from the encapsulin. These data suggest that encapsulins are taken up in macrophages, end up in lysosomes, are broken down, but do sufficiently protect their cargo which in turn ends up in the cytoplasm. This also demonstrated the potential for encapsulin as a drug delivery cargo system and could be used in enzyme therapy.

All these aspects combined show that encapsulins have the potential to be used as drug or vaccine carrier, but can potentially also be used for other applications as well. However, these are just the initial steps. Further research is required to create encapsulins with targeting loops and analyse their specificity, to further study the internalization of encapsulins and to test different, medical cargo. In this thesis the fundamentals of encapsulins are explored and this thesis supports additional research. 


\section{Samenvatting}

Encapsulines zijn nanokooien die bestaan uit eiwitten. De ontdekking ervan door Sutter et al. heeft een nieuw onderzoeksveld geopend. Hoewel er nog geen praktische toepassingen voor encapsulines zijn, is de potentie duidelijk geworden door studies die aantoonden dat encapsulines verschillende eiwitten konden encapsuleren en dat encapsulines gebruikt kunnen worden om therapeutische deeltjes, zoals kleurstoffen en metalen, te transporteren. Vanuit deze basis, die beschreven is in Hoofdstuk 2, hebben we de verdere mogelijkheden van encapsulines onderzocht. Dit staat beschreven in Hoofdstuk 3 tot en met 6 .

We beginnen met de karakterisatie van encapsulines. Hoewel de structuur van de Thermotoga maritima encapsulines (TmE) bekend was en er significante overeenkomsten zijn in de genetische code van verschillende encapsulines, observeerden wij dat de structuur van Brevibacterium linens encapsulines (BlE) kleine verschillen vertoont ten opzichte van TmE. Met cryo-EM hebben we een 3D map gemaakt met een bijna atomaire resolutie van het $\sim 20 \mathrm{~nm}$ grote deeltje, hetgeen staat beschreven in Hoofdstuk 3. Hier ontdekten we dat hoewel de encapsulines zijn opgebouwd uit dimeren, we ook trimer konden onderscheiden. Bovendien lijkt de Eloop de grootte en structuur van het nanodeeltje te beïnvloeden. Tevens vonden we lussen aan de buitenkant van BlE, die mogelijk gemodificeerd kunnen worden om verschillende functionele moleculen aan te verbinden. 
Hoewel we geen nieuwe functionele groepen aan de buitenkant van BlE hebben geplaatst, hebben we dit wel gedaan door aan de buitenkant van TmE chemisch een trypsine remmer te koppelen (Hoofdstuk 6). Dit toonde de potentie aan van het modificeren van encapsulines om ze een nieuwe functionaliteit te geven, hetgeen gebruikt kan worden voor specifieke targeting, diagnostisering en stabilisering.

De modificatie van encapsulines bleef niet gelimiteerd tot de buitenkant. Er was al aangetoond dat encapsulines ook niet-natuurlijke eiwitten konden bevatten, maar medisch relevante eiwitten waren nog niet getest. In Hoofdstuk 4 staat de modificatie van BlE met als nieuwe lading Lasparaginase (ASNase) beschreven. Het enzym bleef actief, maar de activiteit was verminderd. Dit komt waarshijnlijk door de hoge dichtheid van de encapsuline kooi die om de ASNase heen zat, zoals beschreven in Hoofdstuk 3, waardoor de transport van het product, L-aspartaat, gelimiteerd was. Hoewel dit dichte omhulsel de lading beschermt (Hoofdstuk 5), lijkt het ook de activiteit van de enzym-lading te beïnvloeden. De BlE-ASNase heeft ook een biologisch effect; acute lymphoblastische leukemie cellen gingen dood na toedieding van BlE-ASNase. Dit effect was niet zo groot als bij het meer poreuze op CCMV-gebaseerde capside met ASNase. Andere enzymen, die gebruikt kunnen worden voor andere (medische) toepassingen, zouden ook gemaakt, geëncapsuleerd en bestudeerd kunnen worden.

Om encapsulines te gebruiken in medische toepassingen is het belangrijk om een gedetailleerd begrip van hun interacties met cellen te hebben. Dit domein van het onderzoek naar encapsulines was nog niet goed onderzocht. In Hoofdstuk 5 tonen we de eerste studies naar de opname van encapsulines in cellen en de mogelijke immunogeniciteit van encapsulines. Niet alle cellen nemen encapsulines op; in zowel HeLa cellen als C2C12 cellen was er geen opname of was er heel lage opname bij een relatief 
hoge concentratie encapsulines, terwijl macrofagen wel hoge opname bij lagere concentraties toonden. Dit toonde de mogelijkheid om encapsulines te gebruiken voor het specifiek toedienen van medicatie. De opname van encapsulines is laag in cellen die niet macrofagen zijn en dit kan resulteren in verminderde bijwerkingen als encapsulines gebruikt zouden worden om medicijnen te transporteren.

Ook bleken de encapsulines te zorgen voor een immuun reactie, aangezien we M1 differentiatie aantroffen in RAW264.7 macrofagen die behandeld waren met encapsulines. Hoewel dit de efficientie van encapsulines als transportsysteem voor medicijnen zou verminderen, kan het wel voordelig zijn als de encapsulines gebruikt zouden worden voor het toedienen van vaccins.

Tijdens deze celstudies bleek ook dat de lading van de encapsulines, in dit geval het fluorescente mTFP, niet afgebroken werd door de macrofagen, zelfs na 48 uur incubatie nadat ze uit de encapsulines waren gekomen. Dit suggereert dat de de encapsulines, met lading, in de lysosomen terecht komen en daar worden afgebroken, waarna de lading (mTFP) in het cytoplasma terecht komt. Ook deze resultaten bevestigen de potentie van encapsulines om als transportsysteem voor medicijnen te functioneren en om eiwitten specifiek toe te dienen. Dit kan gebruikt worden in bijvoorbeeld enzyem therapie.

Al deze aspecten gecombineerd laten zien dat encapsulines de potentie hebben om as transportmiddel te functioneren, maar dat er mogelijk ook andere toepassingen zijn. Dit zijn echter wel slechts de eerste stappen. Meer vervolgonderzoek is nodig om encapsulines te maken met modificaties aan de buitenkant die ervoor zorgen dat specifieke cellen getarget kunnen worden. Deze complexen moeten dan geanalyseerd worden om hun specificiteit en opname van hun lading in kaart te brengen. Hiervoor 
kunnen ook verschillende ladingen die (medisch) relevant zijn gekozen worden. In deze thesis staat de fundametele informatie die als bouwsteen kan dienen voor dit verdere onderzoek. 


\section{List of Abbreviations}

ABTS

ALL

AMP

asn

ASNase

asp

$\mathrm{Bl}$

$\mathrm{BlE}$

${ }^{\circ} \mathrm{C}$

CAM

CCMV

CD

$\mathrm{CP}$

DAPI

DLS

DMEM

DMSO

DNA

DNAse

DOL

DyP

$\mathrm{EC}_{50}$

ELISA 2,2'-azinobis(3-ethylbenzo-6-thiazolinesulfonic acid) acute lymphoblastic leukemia ampicillin L-asparagine L-asparaginase L-aspartate Brevibacerium linens Brevibacterium linens encapsulin degree Celsius chloramphenicol cowpea chlorotic mottle virus cluster of differentiation capsid protein 4',6-diamidino-2-phenylindole dynamic light scattering Dulbecco's Modified Eagle's Medium dimethylsulfoxide deoxyribonucleic acid deoxyribonuclease degree of labeling dye-decolourizing peroxidase half maximal effective concentration enzyme-linked immunosorbent assay 
EM

EMCS

FBS

FLP

FPLC electron microscopy

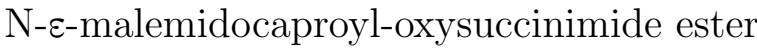
fetal bovine serum ferritin-like protein fast protein liquid chromatography FSC Fourier shell correlation (EM-microscopy) or forward scatter (flow cytometry)

GFP green fluorescent protein

IL

IMEF iron-mineralizing encapsulin-associated firmicute IPTG isopropyl $\beta$-D-1-thiogalactopyranoside L-BApNA

LPS $\mathrm{N} \alpha$-Benzoyl-L-arginine 4-nitroanilide hydrochloride MALDI-TOF matrix assisted laser desorption/ionization time-of-flight (m)EETI-II (modified) Ecballium elaterium trypsin inhibitor II MWCO

molecular weight cut-off

$\mathrm{MxE}$

NHS

NMR

OD

PBS

PCR

$\mathrm{PDB}$

PEG

PfE

PI

PSS

RGD

$\mathrm{Rj} \mathrm{E}$

RNA

RNAse
Myocococcus xanthus encapsulin

$N$-Hydroxysuccinimide nuclear magnetic resonance optical density phosphate buffered saline polymerase chain reaction protein data bank polyethylene glycol Pyrococcus furiosus encapsulin propidium iodide polystyrene sulfonate arginine-glycine-aspartate Rhodococcus jostii encapsulin ribonucleic acid ribonuclease 
ROS

rpm

RT

SCC reactive oxygen species rotations per minute room temperature squamous cell carcinoma

SDS-PAGE sodium dodecyl sulfate polyacrylamide gel electrophoresis SEC

$\mathrm{SSC}$

TEM

(m)TFP

Tm

$\mathrm{TmE}$

TSP

UV

VLP

WGA

\author{
size exclusion chromatography
side scatter \\ size exclusion chromatography
side scatter \\ transmission electron microscopy \\ (monomeric) teal fluorescent protein \\ Thermotoga maritima \\ Thermotoga maritima encapsulin \\ (trimethylsilyl)propionic-2,2,3,3-d4 acid \\ ultraviolet \\ virus-like particle \\ wheat germ agglutinin
}




\section{Acknowledgements}

Dit is het laatste stukje dat ik schrijf. Tevens het meest gelezen stukje. En dat terwijl er in de rest van dit boekje veel meer tijd en moeite is gaan zitten. Maar dat betekent niet dat dit een snel afgeraffeld stukje zonder enige relevantie is. Sterker nog, het betreft een dankbetuiging aan een groep zonder wie dit boekje er helemaal niet was geweest. Mijn dank gaat naar iedereen die mij heeft geholpen, bijgestaan, geadviseerd en mijn PhD-tijd zeer aangenaam heeft gemaakt. Daar horen zeker een aantal personen bij die speciale dank verdienen.

Ten eerste mijn promotor, Jeroen Cornelissen. Veel dank voor je begeleiding tijdens de afgelopen 4 jaar. Je inzichten, suggesties en hulp tijdens het hele proces waardeer ik zeer.

I also thank the members of my committee for participating in my defense and commenting on my concept thesis. Prof. dr. Heddle, prof. dr. Kros and prof. dr. Passier, many thanks for being part in this part of my career. Prof. dr. ir. Pascal Jonkheijm en dr. ir. Jos Paulusse dank ik, behalve voor hun deelname in mijn commissie, ook voor hun suggesties en adviezen tijdens de colloquia, in de wandelgangen en aan de koffietafel. 
I also thank the other academic members of our group, prof. dr. ir. Jurriaan Huskens, prof. dr. ing. Nathalie Katsonis, dr. Tibor Kudernac and dr. Wim Verboom, whose advice and questions helped improve my work and this thesis.

Tijdens mijn $\mathrm{PhD}$ (en net zo significant na het officiële einde van mijn contract) ben ik ook ontzettend goed bijgestaan door Nicole en Izabel. Jullie deskundigheid in het navigeren van universiteitsbureaucratie heeft er mede voor gezorgd dat ik, ten tijde van schrijven, nog geen grijze haren heb.

Ik ben ook veel dank verschuldigd aan de geweldige groep technicians. Richard, Marcel, Regine en Bianca; heel erg bedankt voor al jullie hulp met apparatuur, chemicaliën, reacties, en nog meer. Het was ontzettend fijn om in de laboratoria van BNT en MnF te werken en dat komt zeker door jullie harde werk om alles goed draaiende te houden.

Dit geldt ook voor het BioNL. Ine, veel dank voor het soepel laten verlopen van alles wat er omgaat in het BioNL.

Nicole and Melissa, thank you for being my paranymphs. You are great friends of mine and I am very happy and privileged to have met you. Nicole, thank you for the movie-nights. I really enjoyed those. My best wishes to you with your own thesis. I look forward to reading it! Melissa, I wish you the best of luck with new adventures careerwise and also a lot of happiness with Mike. Thanks as well for the many boardgames we played over the years.

A significant part of this thesis is the result of a very fruitful collaboration with the group of prof. dr. Castón from Madrid. Pepe, many thanks for your help with the various projects, for facilitating my visit to 
Madrid, and your friendship. David, thank you as well for your help in all the cryo-EM experiments. Caroline, thank you for your hard work with the cryo-EM. The images and data look amazing and I was very pleased with our collaboration. Good luck on finishing your thesis as well!

During my PhD I supervised 10 students, either bachelor or master, which was a great joy to do. I thank Berrie, Christa, Emma, Janita, Joris, Mark, Victoria, Alexander, Maria and Mateusz for their great work.

Mark! Mede door jou (en whisky) ben ik in Enschede beland. Gedurende onze lange vriendschap hebben we al veel meegemaakt. Het was ook heel fijn dat jij al in de groep met je $\mathrm{PhD}$ bezig was; ik had aan jou een goede houvast. Bovendien hebben we door de jaren heen nog een hoop samengewerkt. Veel dank voor alles de afgelopen jaren! En ontzettend veel plezier en succes met je eigen werk, maar vooral (samen met Laura) met Olen.

Erik, we hebben veel samen opgetrokken. Nog meer toen je in hetzelfde appartementencomplex (doch zover mogelijk van mijn huis af) kwam wonen. Tijdens koffiepauzes en daarbuiten hebben we interessante gesprekken gehad die mij altijd deden nadenken. Samen de cursus machine learning doen was ook heel leuk, net als de bordspelletjes. Veel dank daarvoor. Heel veel succes met het laatste stukje van je PhD. Ook heel veel geluk samen met Elvera, van de aankomende bruiloft tot lang daarna! Elvera, jij ook bedankt voor je vriendschap! Mijn gelukwensen gelden natuurlijk net zo veel voor jou.

Luca, many thanks for your friendship during my PhD. And for the many boardgames we played. And also for the beautiful cover you designed. Your photoshop skills are fantastic (promise to use your powers for good). Best of luck with your thesis! Hantanyel! 
Samen met Erik, Luca, Melissa, dank ik ook Nico voor de Pathfinder avonden. Het bood mij de mogelijkheid om echt mezelf te zijn: een halfork met lage intelligence en charisma. Veel geluk samen met Bea, en veel succes met de laatste stappen van je $\mathrm{PhD}$.

Jenny! You joined BNT on the same day as I did. Thank you for your friendship, your delicious food, your help during my $\mathrm{PhD}$ and your happiness! You will also soon be done with the thesis! Good luck with the last bits. Jacopo, you also joined the same day as me. It was great learning the ins and outs of working in Enschede together with you. Thank you especially in the last part, for being a great "roommate".

Rindia, thank you as well. You were there during my first years as a PhD-student. My first projects were together with you. Thank you for your guidance and advice.

Tijdens de PhD heb ik ook veel kunnen optrekken met de "Eetgroep". Stan, Wouter, Rianne, Janneke, Milou, Rik, en Mark, veel dank voor de gezelligheid tijdens de etentjes en de pauzes.

This joy during my $\mathrm{PhD}$ is also thanks to everyone from the BNT and MnF group that is or was present. Thank you all for the great work environment.

Mart, dank dat je al jaren mijn vriend bent, en dank voor de mooie foto van de encapsulin die nu op de cover staat. Ook dank voor het faciliteren van het whisky-avondje wat resulteerde in mijn aanstelling aan de UT. Samen met de rest van de groep (Peter, Jeroen, Mark en Falco) zijn we al jaren vrienden en ik hoop dat dat nog jaren zo blijft. 
Maike, ontzettend bedankt voor je goede vriendschap en dat ik altijd met je kan praten over van alles (met name boeken). Jouw vriendschap is me heel veel waard.

Jordy en Kim, Lennart en Mariska, Pa en Ma. Heel erg bedankt voor alles. Van 2 november 1991 tot nu. Bedankt dat jullie er altijd voor me zijn, dat ik bij jullie terecht kan voor alle leuke en minder leuke dingen. Zo blijkt dat een dankwoord nog best lastig is, want ik kan niet de juiste woorden vinden om mijn dank naar jullie goed te verwoorden. Ik houd van jullie! 


\section{About the Author}

Robin Klem was born November $2^{\text {nd }}$, 1991 in Wageningen, the Netherlands. After obtaining his bachelor degree in Biopharmaceutical Sciences at Leiden University in 2012, he continued with the master program. He obtained his master degree in Biopharmaceutical Sciences in 2014 cum laude. He performed and wrote his master thesis under the supervision of Prof. dr. W. Jiskoot and dr. P. Meij at the department of Drug Delivery Technology, Leiden Academic Centre for Drug Research, Leiden University and the department of Hematology at the Leiden University Medical Centre. The thesis was entitled "Flow Imaging Microscopy Techniques to Assess the Quality of Cell Therapy Products".

He continued working at BBB Therapeutics in Leiden after which he started as a PhD candidate at the University of Twente in the Biomolecular Nanotechnology group under the supervision of Prof. dr. Jeroen J. L. M. Cornelissen in 2015. The aim of the research project was to further study the possibilities and uses of the bacterial protein cage encapsulin, the results of which are described in this thesis. 


\section{List of Publications and Presentations}

\section{List of Publications}

R. Klem, M. V. de Ruiter, V. M. Dombrowe, A. Juan, D. Gil-Cantero, C. Allende-Ballestero, D. Luque, J. R. Castón, J. J. L. M. Cornelissen; "Construction and Evaluation of Asparaginase-loaded Protein Cages as Nanomedicine", manuscript in preparation

C. Allende-Ballestero, R. Klem, D. Luque, J. J. L. M. Cornelissen, J. R. Castón; "Structural Analysis of Brevibacterium linens Encapsulin", manuscript in preparation

S. Longo, M. Marcinek, R. Klem, J. J. L. M. Cornelissen, P. Jonkheijm, J. Huskens; "Encapsulin as Multivalent Nanoplatform Displaying Ligands with Precise Spatial Control", manuscript in preparation

M. V. de Ruiter, R. Klem, D. Luque, J. J. L. M. Cornelissen, J. R. Castón; "Structural Nanotechnology: Three-dimensional Cryo-EM and its use in the Development of Nanoplatforms for in Vitro Catalysis", Nanoscale 2019, 11, 4130 - 4146 
R. Klem, M. V. de Ruiter, J. J. L. M. Cornelissen; "Protecting Encapsulin Nanoparticles with Cysteine-Knot Miniproteins", Mol. Pharm. 2018, 15, 2991 - 2996

A. S. Sediq, R. Klem, M. R. Nejadnik, P. Meij, W. Jiskoot; "Label-Free, Flow-Imaging Methods for Determination of Cell Concentration and Viability", Pharm. Res. 2018, 35, 150

R. M. Putri, C. Allende-Ballestero, D. Luque, R. Klem, K. A. Rousou, A. Liu, C. H.-H. Traulsen, W. F. Rurup, M. S. T. Koay, J. R. Castón, J. J. L. M. Cornelissen; "Structural Characterization of Native and Modified Encapsulins as Nanoplatforms for in Vitro Catalysis and Cellular Uptake", ACS Nano 2017, 11, 12796 - 12804

R. Torosantucci, D. Weinbuch, R. Klem, W. Jiskoot; "Triethylenetetramine prevents insulin aggregation and fragmentation during copper catalyzed oxidation", Eur. J. of Pharm. and Biopharm. 2013, 84, 464 471.

\section{List of Presentations}

R. Klem, M. V. de Ruiter, V. M. Dombrowe, A. J. R. del Valle, J. Castón, J. J. L. M. Cornelissen; Enzyme Loaded Protein Cages as Nanomedicine in Leukemia Treatment, GRS/GRC Physical Virology 2019, Ventura, United States of America. Poster

R. Klem, M. V. de Ruiter, V. M. Dombrowe, A. J. R. del Valle, J. Castón, J. J. L. M. Cornelissen; Enzyme Loaded Protein Cages as Nanomedicine in Leukemia Treatment, CHAINS 2018, Veldhoven, the Netherlands. Oral and Poster 
R. Klem, M. V. de Ruiter, V. M. Dombrowe, A. J. R. del Valle, J. J. L. M. Cornelissen; In Vitro Activity of L-Asparaginase Encapsulated in B. linens Encapsulin or Cowpea Chlorotic Mottle Virus Nanoparticles, ESCDD 2018, Egmond aan Zee, the Netherlands. Poster

R. Klem, R. M. Putri, C. Allende-Ballestero, D. Luque, K. Rousou, A. Liu, C. H.-H. Traulsen, W. F. Rurup, M. S. T. Koay, J. R. Castón, J. J. L. M. Cornelissen; Structural Characterization of Native and Modified Encapsulins as Nanoplatforms for in Vitro Catalysis and Cellular Uptake, CHAINS 2017, Veldhoven, the Netherlands. Poster

R. Klem, M.V. de Ruiter, J.J.L.M. Cornelissen; Exterior Modification of Thermotoga Maritima Encapsulin with Ecballium Elaterium Trypsin Inhibitor II, GRS/GRC Physical Virology 2017, Barga, Italy. Poster

R. Klem, M.V. de Ruiter, J.J.L.M. Cornelissen; Exterior Modification of Thermotoga Maritima Encapsulin with Ecballium Elaterium Trypsin Inhibitor II, CHAINS 2016, Veldhoven, the Netherlands. Poster 
